\title{
An Application of the Coherent Noise Model for the Prediction of Aftershock Magnitude Time Series
}

\author{
Stavros-Richard G. Christopoulos ${ }^{1,2}$ and Nicholas V. Sarlis ${ }^{2,3}$ \\ ${ }^{1}$ Faculty of Engineering, Environment and Computing, Coventry University, Priory Street, Coventry CV1 5FB, UK \\ ${ }^{2}$ Solid Earth Physics Institute, Department of Physics, School of Science, National and Kapodistrian University of Athens, \\ Panepistimiopolis, Zografos, 15784 Athens, Greece \\ ${ }^{3}$ Section of Solid State Physics, Department of Physics, School of Science, National and Kapodistrian University of Athens, \\ Panepistimiopolis, Zografos, 15784 Athens, Greece \\ Correspondence should be addressed to Stavros-Richard G. Christopoulos; strichr@phys.uoa.gr
}

Received 18 July 2016; Accepted 9 October 2016; Published 20 February 2017

Academic Editor: Alicia Cordero

Copyright (C) 2017 Stavros-Richard G. Christopoulos and Nicholas V. Sarlis. This is an open access article distributed under the Creative Commons Attribution License, which permits unrestricted use, distribution, and reproduction in any medium, provided the original work is properly cited.

\begin{abstract}
Recently, the study of the coherent noise model has led to a simple (binary) prediction algorithm for the forthcoming earthquake magnitude in aftershock sequences. This algorithm is based on the concept of natural time and exploits the complexity exhibited by the coherent noise model. Here, using the relocated catalogue from Southern California Seismic Network for 1981 to June 2011, we evaluate the application of this algorithm for the aftershocks of strong earthquakes of magnitude $M \geq 6$. The study is also extended by using the Global Centroid Moment Tensor Project catalogue to the case of the six strongest earthquakes in the Earth during the last almost forty years. The predictor time series exhibits the ubiquitous $1 / f$ noise behavior.
\end{abstract}

\section{Introduction}

The prediction of the magnitudes and occurrence times of aftershocks is of crucial importance for restricting the losses caused by strong earthquakes (EQs, hereafter) because buildings already damaged by the mainshock may collapse upon the occurrence of a strong aftershock. Recently, an algorithm has been suggested [1] on the basis of the coherent noise model [2-4] and natural time [5-7] that might be useful for the determination of both magnitudes and occurrence times of aftershocks. It is the main scope of the present paper to investigate the applicability of such an algorithm to aftershock time series in Southern California. This area has been selected in view of the publication [8] of an accurate waveform relocated EQ catalogue for Southern California from 1981 to June 2011 [9] that exhibits tighter spatial clustering of seismicity than the routinely generated catalogue does.

The coherent noise model [2-4] is a model that shows reorganization events (avalanches) whose size distribution follows a power law over many decades and displays aftershock events. These events have been shown $[3,10,11]$ to exhibit a behavior similar to that of the Omori-Utsu law [12]; see also [13, 14], for real EQ aftershocks. Moreover, it has been recently shown [15] that it is compatible with the unified scaling law [16] of waiting times between EQs.

In a time series comprising $N$ avalanches (or EQs), natural time $\chi_{k}=k / N$ serves as an index for the occurrence of the $k$ th event [5-7]. Natural time focuses on the sequential order of the events and the analysis is usually made by using the pair $\left(\chi_{k}, Q_{k}\right)$, where $Q_{k}$ is a quantity proportional to the size (and hence to the energy) of the $k$ th event. Natural time analysis has found useful applications in a variety of fields: Statistical Physics (e.g., [17-23]), Cardiology (e.g., [24-27]), Geophysics (e.g., [6, 28-32]), Atmospheric Sciences (e.g., [33, 34]), Seismology (e.g., [35-40]), Physics of EQs (e.g., [41-45]), EQ prediction (e.g., $[5,46-52]$ ), and so on (for a recent review see [7]). For the case of EQs [36], $Q_{k} \propto 10^{c M_{k}}$, where $M_{k}$ is the magnitude of the $k$ th EQ and $c \approx 1.5$. The two quantities 
TABLE 1: All EQs with magnitude $M \geq 6.0$ during the period 1981 to June 2011 within the reporting area for local events of SCSN (see the polygon in Figure 1 of Hauksson et al. [8]). The dimensions of the rectangular aftershock area, centered at each epicenter, used in this study are also shown.

\begin{tabular}{lccccc}
\hline EQ name & $M$ & Origin time (UTC) & Lat $\left(^{\circ}\right)$ & Lon $\left(^{\circ}\right)$ & Area considered \\
\hline Landers & 7.30 & $1992 / 06 / 2811: 57: 33$ & 34.202 & -116.435 & $1.1^{\circ} \times 1.1^{\circ}$ \\
El Mayor-Cucapah & 7.20 & $2010 / 04 / 0422: 40: 42$ & 32.264 & -115.295 & $1.0^{\circ} \times 1.0^{\circ}$ \\
Hector Mine & 7.10 & $1999 / 10 / 1609: 46: 43$ & 34.595 & -116.271 & $0.9^{\circ} \times 0.9^{\circ}$ \\
Northridge & 6.70 & $1994 / 01 / 1712: 30: 54$ & 34.206 & -118.549 & $0.6^{\circ} \times 0.6^{\circ}$ \\
2nd Superstition Hills & 6.60 & $1987 / 11 / 2413: 15: 56$ & 33.017 & -115.843 & $0.5^{\circ} \times 0.5^{\circ}$ \\
Big Bear aftershock & 6.30 & $1992 / 06 / 2815: 05: 30$ & 34.202 & -116.828 & $0.3^{\circ} \times 0.3^{\circ}$ \\
lst Superstition Hills & 6.20 & $1987 / 11 / 2401: 54: 14$ & 33.082 & -115.779 & $0.3^{\circ} \times 0.3^{\circ}$ \\
Joshua Tree & 6.10 & $1992 / 04 / 2304: 50: 22$ & 33.968 & -116.313 & $0.3^{\circ} \times 0.3^{\circ}$ \\
\hline
\end{tabular}

$k\left(=N \chi_{k}\right)$ and $M_{k}$ form the basis of the aftershock magnitude prediction algorithm, which is discussed in the next section.

\section{Materials and Methods}

2.1. Data Analyzed. As mentioned in the introduction, we consider the waveform relocated catalogue [8] for Southern California from 1981 to June 2011 [9]. In order to filter out distant poorly constrained events, we use the geographic polygon shown in Figure 1 of Hauksson et al. [8] that covers the Southern California Seismic Network (SCSN) reporting area for local events. The $M \geq 6.0$ events that occurred within this area are shown in Table 1 . As seen in this table, there are 4 stronger EQs with $M \geq 6.7$ which are the 1992 Landers, the 2010 El Mayor-Cucapah, the 1999 Hector Mine, and the 1994 Northridge EQs (see Figure 1), and 4 smaller EQs which are the two Superstition Hills large events [53] in 1987 and the 1992 Joshua Tree and Big Bear events (see Figure 2). The latter two EQs are related $[54,55]$ to the Landers EQ which is the strongest one in California for the last sixty years (e.g., see Table 1 of [56]).

Following Shcherbakov et al. [13], we consider as aftershocks all reported events that occurred during a period $T$ within a region centered at the epicenter of the each strong EQ (see the last column of Table 1). The linear dimension of each region scales with $10^{0.5 M_{m}}$ starting from the region of $1.1^{\circ} \times$ $1.1^{\circ}$ selected by Shcherbakov et al. [13] for Landers EQ (cf. the scaling of the aftershock zone with mainshock magnitude $M_{m}$ was firstly introduced by Utsu and Seki [57]). This scaling has been employed since it allows the determination of the "aftershock" spatial window upon the occurrence of the mainshock and does not make use of any a posteriori information concerning the aftershocks. Hence, the spatial range of the space-time window usually called Omori regime-which is identified [58] by examining the best fits of the Omori-Utsu law to the aftershock data-may differ from this window. The period $T$ examined was one year for the four stronger mainshocks while it varies for the four smaller EQs (see Results and Discussion).

2.2. Coherent Noise Model and the Algorithm. The events, to be called avalanches, in the coherent noise model result from the following procedure $[2,3]$. Consider a system of $N_{a}$ agents; for example, the points of contact in a subterranean fault, for each agent $i$ we associate a threshold $x_{i}, i=$ $1,2, \ldots, N_{a}$ that represents the amount of stress that an agent withstands before it breaks. Without loss of generality [3], $x_{i}$ may come from the uniform distribution in the interval $0 \leq x<1$. The dynamics of the model consists of two steps, a "stress" step, which is more important and sufficient to produce large avalanches, and an "aging" step. During the "stress" step, we select a random number (or "stress" level) $\eta$ from some probability distribution function $p_{\text {stress }}(\eta)$ and replace all $x_{i}$ that are smaller than $\eta$ with new values resulting from the uniform distribution in the interval $0 \leq x<1$. The number of agents whose thresholds are renewed is the size $S$ of the avalanche. During the "aging" step, a fixed fraction $f$ of agents is selected at random and their thresholds are also replaced with new thresholds obtained again from the uniform distribution in the interval $0 \leq x<1$. If we assume that $N_{a} \rightarrow \infty$, the thresholds $x_{i}$ are represented by a threshold distribution function $p_{\text {thres }}(x)$ which initially $(k=0)$ is considered uniform in the interval $0 \leq x<1$; that is, $p_{\text {thres }}^{(0)}(x)=1$ (see Figure 3 ). The size $S_{1}$ of the first avalanche $(k=1)$ is just the probability $\operatorname{Prob}\left[x<\eta_{1}\right]=$ $\int_{0}^{\eta_{1}} p_{\text {thres }}^{(0)}(x) d x=\eta_{1}$ which represents the "mass" of the agents that had thresholds smaller than $\eta_{1}$. After the subsequent "aging" step the threshold distribution becomes $p_{\text {thres }}^{(1)}(x)$. Upon repeating the two steps for the second time using $\eta_{2}$ we can obtain the size $S_{2}$ of the second avalanche and the new threshold distribution $p_{\text {thres }}^{(2)}(x)$ and so on.

In the case of the coherent noise model for $N_{a} \rightarrow \infty$, the threshold distribution $p_{\text {thres }}^{(k)}(x)$ after the $k$ th avalanche is a piecewise constant function having [1] the following general form:

$$
p_{\text {thres }}^{(k)}(x)=\sum_{n=0}^{n_{k}} d_{n}^{(k)} \Theta\left(x-b_{n}^{(k)}\right),
$$

where $n_{k}$ is the number of steps present in the threshold distribution function after the $k$ th avalanche (e.g., see Figure 3 ), $b_{0}^{(k)}=0, b_{n}^{(k)}$, and $d_{n}^{(k)}$ are positive real parameters, and $\Theta(x)$ is the Heaviside (unit) step function; that is, $\Theta(x)=0$ if $x<0$ and $\Theta(x)=1$ if $x \geq 0$. The analysis of numerical results has 

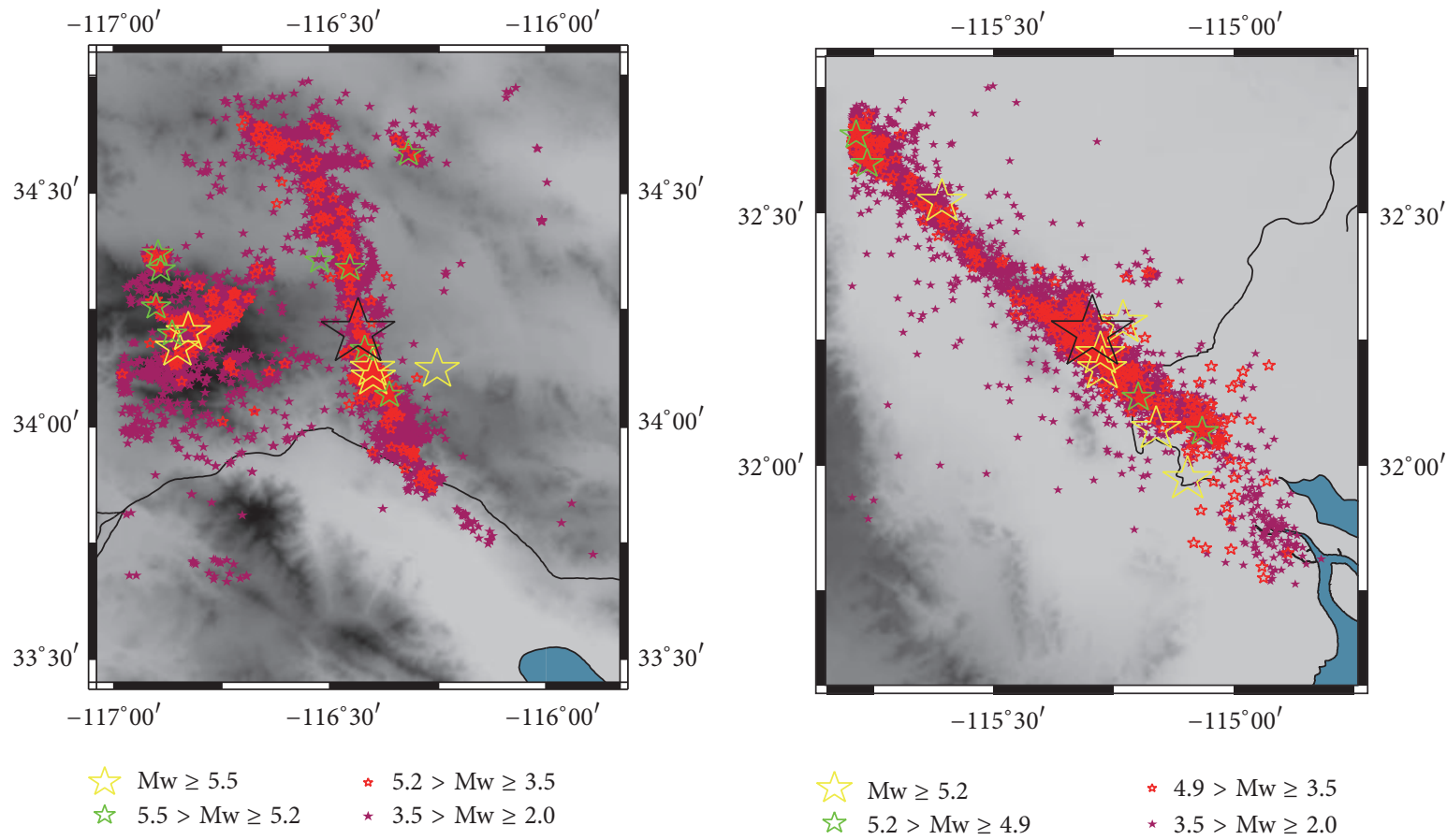

(a)

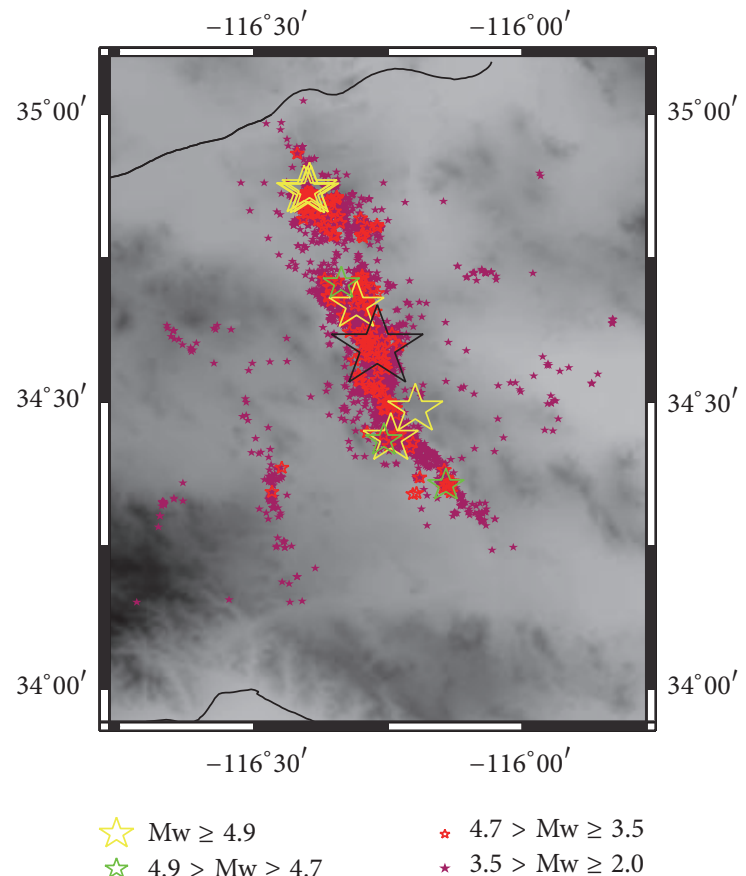

(c)

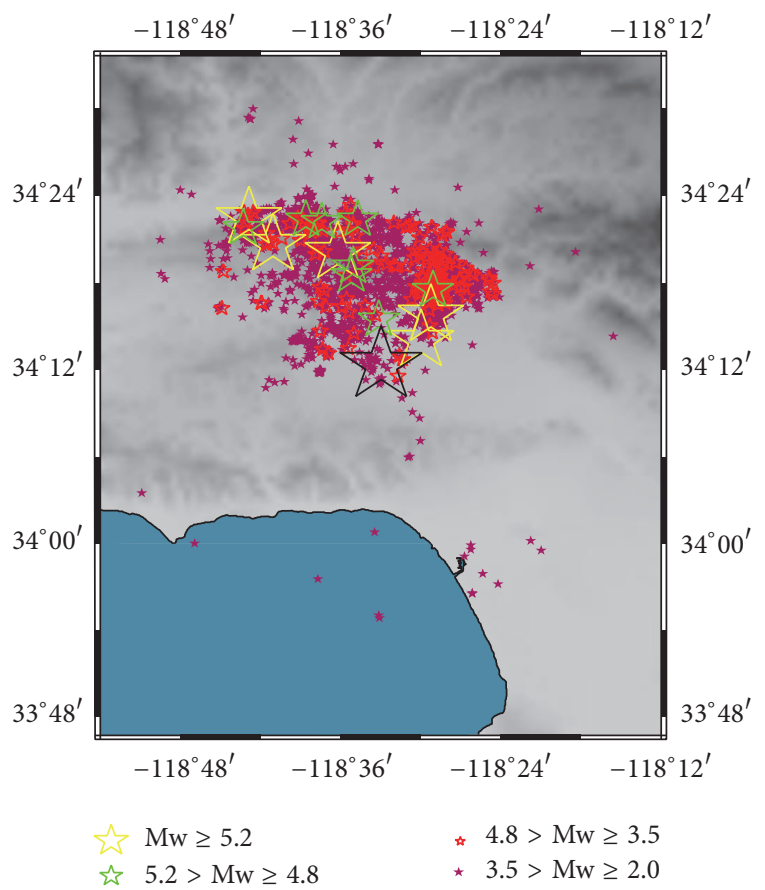

(d)

FIgURE 1: Map of the aftershocks considered (see Table 1) in the case of (a) Landers, (b) El Mayor-Cucapah, (c) Hector Mine, and (d) Northridge EQs whose epicenters are depicted by the largest (black) stars, respectively.

shown (e.g., see Figure 5 of [1]) that the integer number $n_{k}$ may serve as a predictor for the size $S_{k+1}$ of the next avalanche. It has been also found [1] that $n_{k}$ as a function of $k$ (and hence versus natural time) exhibits the ubiquitous $1 / f$ noise [5963] (together with a logarithmic trend, e.g., see Eqs. (31) and (32) of [1]).
The number of steps $n_{k}$ changes when a "stress" level $\eta$ is applied: (a) eliminating at least two smaller "stress" levels previously applied (cf. the case $k=3$ in Figure 3) or (b) being smaller than the smaller nonzero $b_{n}^{(k)}$ (cf. the case $k=2$ in Figure 3). When all $\eta_{k}<1, n_{k}$ coincides with the cardinality $\left|E_{k}\right|$ of the sets $E_{k}$ of successive extrema formed when 


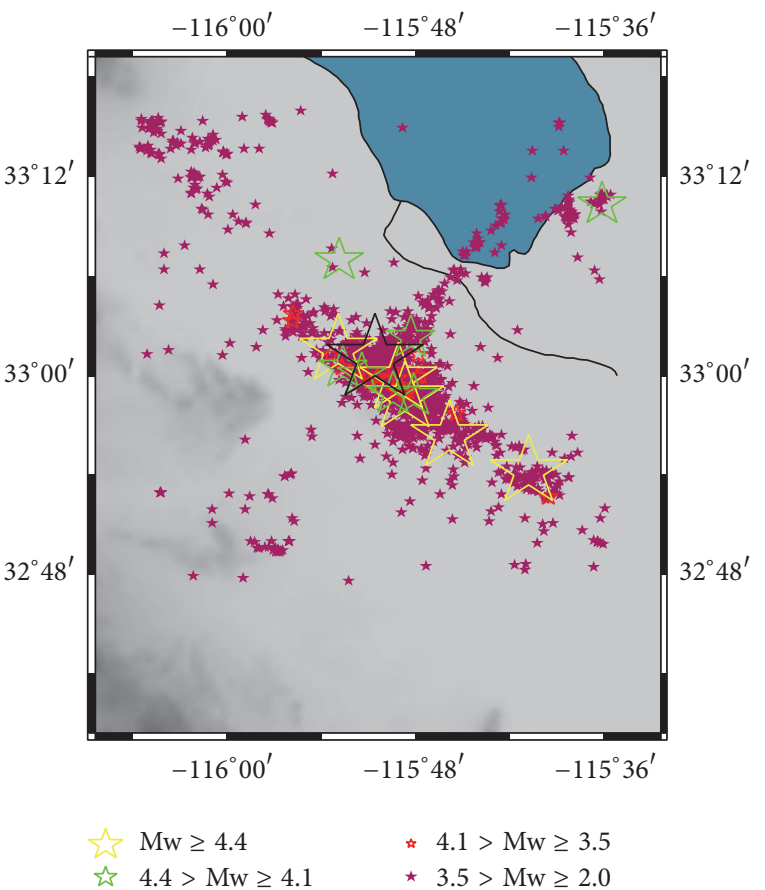

(a)

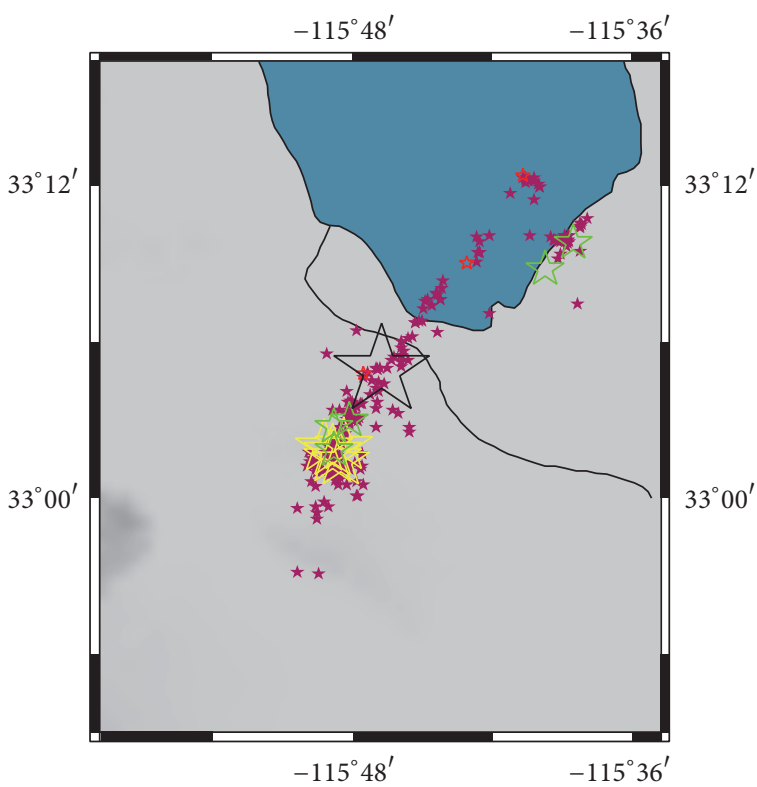

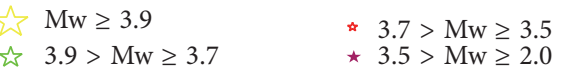

(c)

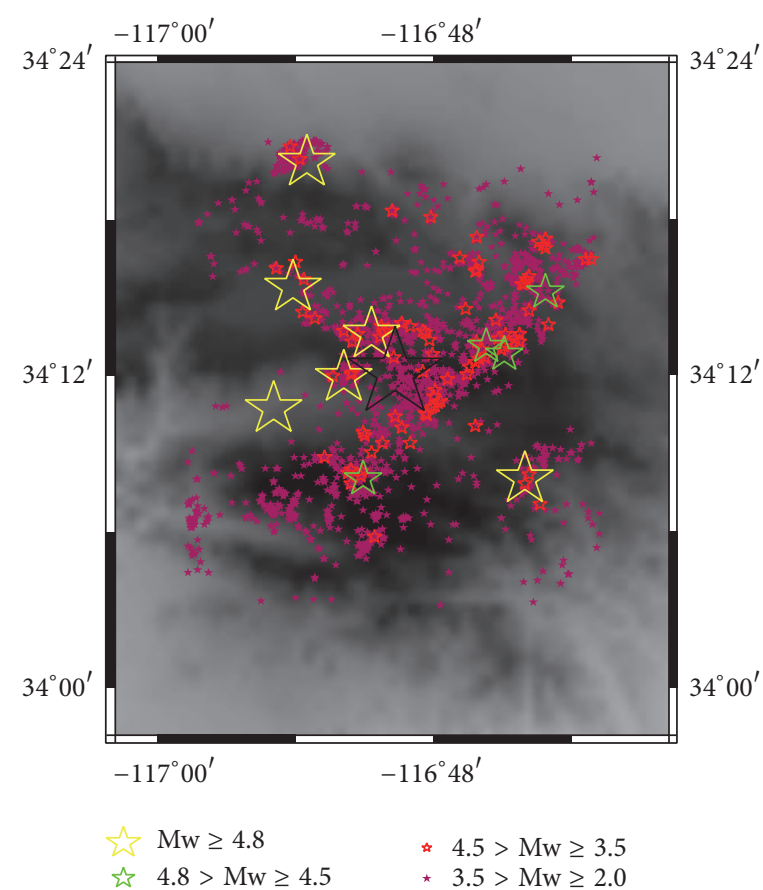

(b)

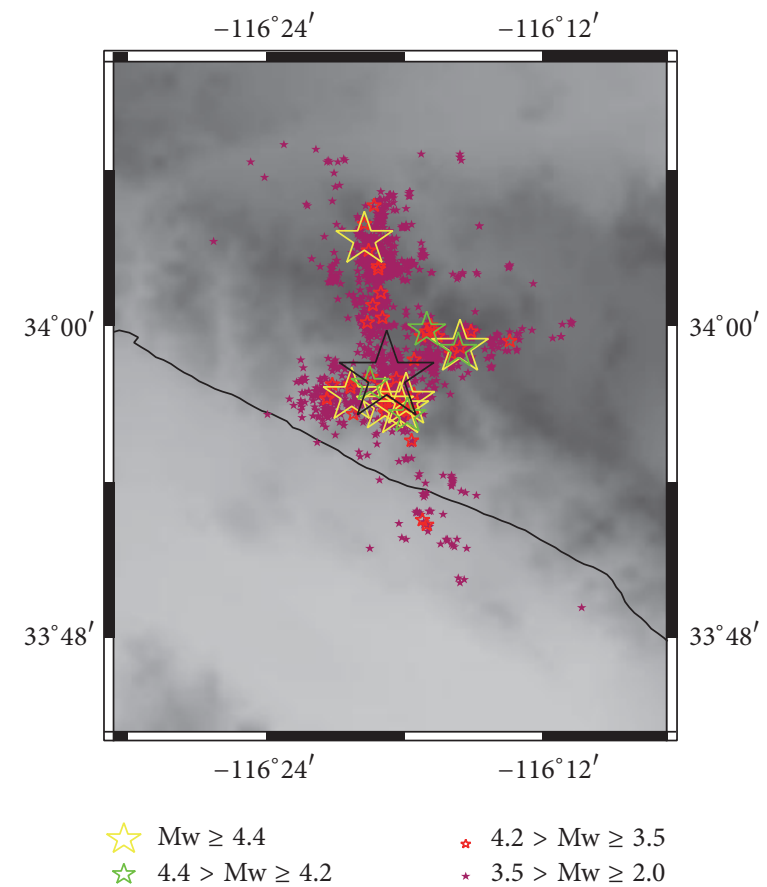

(d)

FIGURE 2: Map of the aftershocks considered (see Table 1) in the case of (a) 2nd Superstition Hills, (b) Big Bear aftershock, (c) 1st Superstition Hills, and (d) Joshua Tree EQs whose epicenters are depicted by the largest (black) stars, respectively.

studying the time series of the random stresses $\eta_{k}$. The sets $E_{k}$ of successive extrema are defined [25] as follows: $E_{0}$ equals the empty set $\emptyset$. Each subsequent $E_{k}$ is obtained by the procedure described below for $k$ times: select a random number $\eta_{k}$ from a given probability density function $f(\eta)$ and compare it with all the members of $E_{k-1}$. In order to construct the set $E_{k}$, we discard from the set $E_{k-1}$ all its members that are smaller than $\eta_{k}$ and furthermore include $\eta_{k}$. Thus, $E_{k} \neq \emptyset$ for all $k>0$ and $E_{k}$ is a finite set of real numbers whose members are always larger than or equal to $\eta_{k}$. Moreover, $\max \left[E_{k}\right] \geq \max \left[E_{k-1}\right]$. 


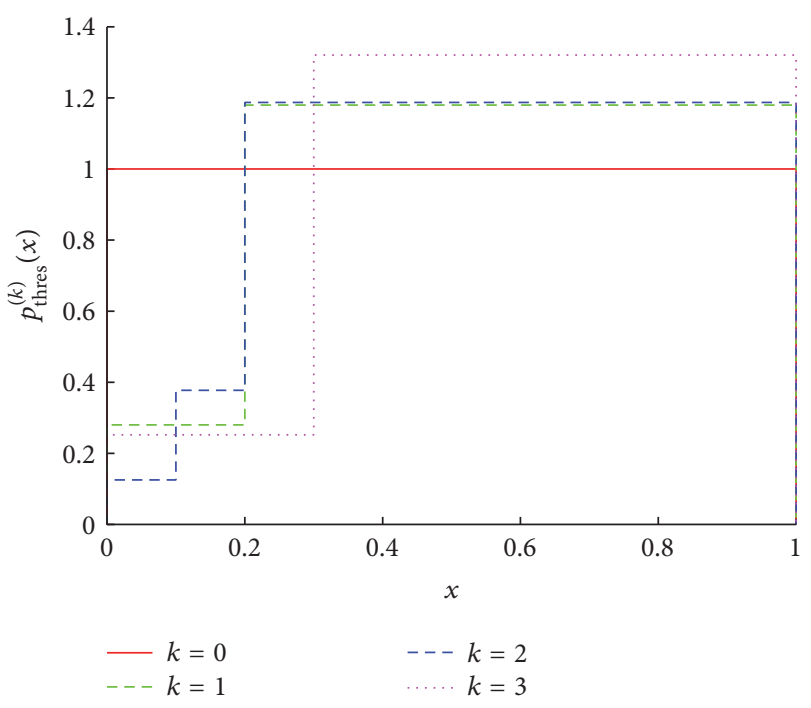

FIGURE 3: Schematic diagram of the threshold distribution function $p_{\text {thres }}^{(k)}(x)$ after the $k$ th avalanche in the coherent noise model with $N_{a} \rightarrow \infty$ : for $k=0$ (solid red), $k=1$ (dashed green) exhibiting a single step at $x=0.2, k=2$ (dashed blue) with two steps at $x=0.1$, 0.2 , and $k=3$ (dotted magenta) with a single step at $x=0.3$. The stress levels $\eta_{1}=0.2, \eta_{2}=0.1$, and $\eta_{3}=0.3$ have been applied during the first, second, and third "stress" step, respectively. The value of $f=0.1$ has been used.

The increase of the cardinality $\left|E_{k}\right|$ of these sets is at the most 1 , but its decrease may be as large as $\left|E_{k}\right|-1$, thus exhibiting a characteristic asymmetry as $k$ increases.

The aforementioned predictability of the coherent noise model on the basis of $n_{k}$ could be also seen as follows: when a relatively large random stress $\eta_{k}$ is applied, the coherent noise model almost "forgets" the effect of previously applied smaller stresses acquiring relatively high threshold density for a relatively wide threshold range starting from $x=0$ (cf. the case $k=3$ for $x<0.3$ in Figure 3). Since the archetypal physical system of the coherent noise model assumed (e.g., see the last few lines of the first page of [2]) that the agents correspond to the points of contact in a subterranean fault, it has been suggested [1] that the coherent noise model predictability can be exploited in the case of EQ aftershocks. In the latter case, one studies the successive extrema formed in the time series of the aftershock magnitudes $M_{k}\left(M_{k} \geq M_{\text {thres }}\right.$, where $M_{\text {thres }}$ is a threshold magnitude) as a function of their order of occurrence $k$, that is, natural time, by assuming $\eta_{k}=M_{k} / 10$. Setting $k=0$ at the occurrence time of the mainshock, as shown in the upper left rectangular box of Figure 4, the corresponding sets $M_{k}$ of the successive extrema (as defined above) in the aftershock magnitude time series are constructed and the cardinality $e_{k} \equiv\left|\mathrm{M}_{k}\right|$ (e.g., see Figure 4) plays a role similar to that of $n_{k}$ in the case of the coherent noise model. A simple computer program that calculates $e_{k}$ is presented in Appendix.

Figure 5(a) depicts the aftershock magnitude time series of the Landers EQ (see Table 1) versus conventional time together with the time series of $e_{k}$. The argument of the latter time series has been intentionally shifted by unity in order to

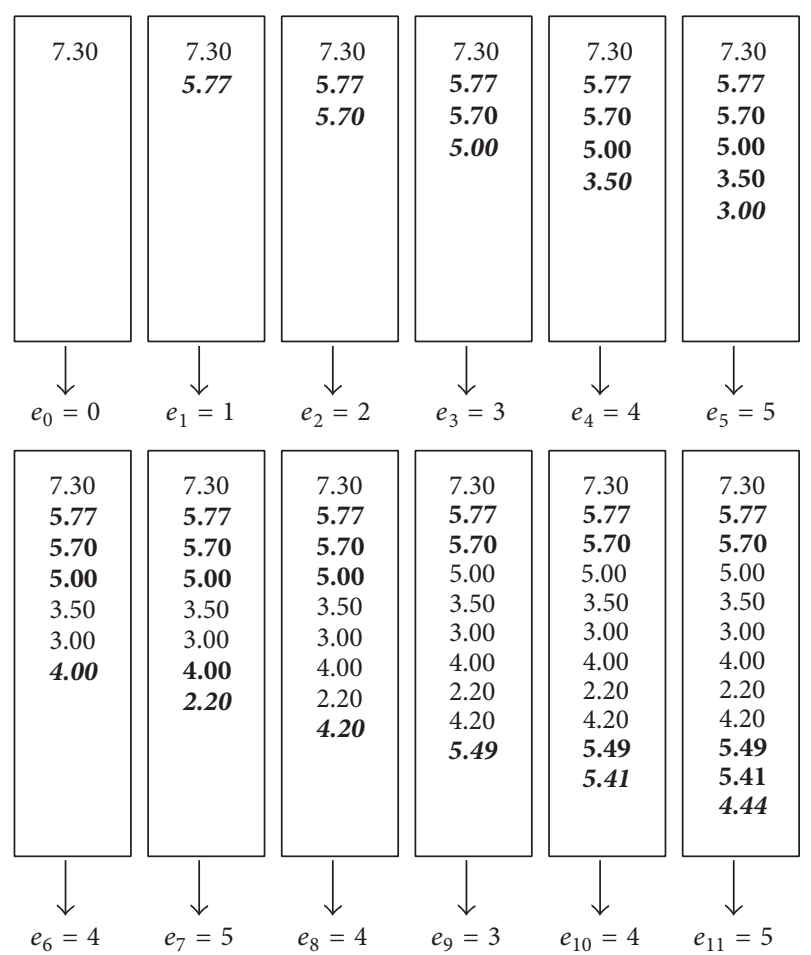

FIGURE 4: How the sets $M_{k}$ of the successive extrema of aftershocks are formed in the case of the Landers EQ. In each rectangular box the aftershocks with $M_{k} \geq M_{\text {thres }}$ are arranged vertically according to their order of occurrence. Each time a new aftershock $M_{k}$ (shown in italics) takes place, the members of $\mathrm{M}_{k}$ (shown in bold) are obtained by discarding all the previous members, that is, those of $\mathrm{M}_{k-1}$, that are smaller than $M_{k}$. The number of elements of $\mathrm{M}_{k}$ is the cardinality $\left|\mathrm{M}_{k}\right|$ denoted by $e_{k}$.

compare the value of $e_{k-1}$, that is, the number of successive extrema before the occurrence of the $k$ th aftershock, with the magnitude $M_{k}$ of the $k$ th aftershock. We observe a characteristic pattern which reflects the already mentioned asymmetry when $e_{k}$ is considered as time series with respect to the natural number $k$, but no clear correlation between $M_{k}$ and $e_{k-1}$ can be seen. For this reason, we plot in Figure 5(b) the excerpt corresponding to the first three and a half hours after the mainshock occurrence. During this period, the strongest aftershock of Landers EQ (see the sixth line of Table 1), the Big Bear aftershock [54], took place. We may observe that most of the time strong aftershocks occur when $e_{k-1}$ is below 5. In order to further clarify what happens, it is most appropriate to depict the results in natural time. This is done in Figure 5(c) where we observe that the Big Bear aftershock occurs when $e_{k-1}$ was equal to 4 . Thus, we observe that for Landers EQ the suggestion that $e_{k-1}$ might be a useful predictor could be considered plausible. The subject of the next section is to examine statistically whether $e_{k-1}$ can be used for the prediction of the magnitude of the forthcoming aftershock, in the sense that this magnitude exceeds a threshold. Here, we note that the coherent noise model method might be able to predict the magnitude of the next aftershock; however it cannot say when it will occur. This makes impossible the use of other verification methods as the Molchan diagram [64-66]. 


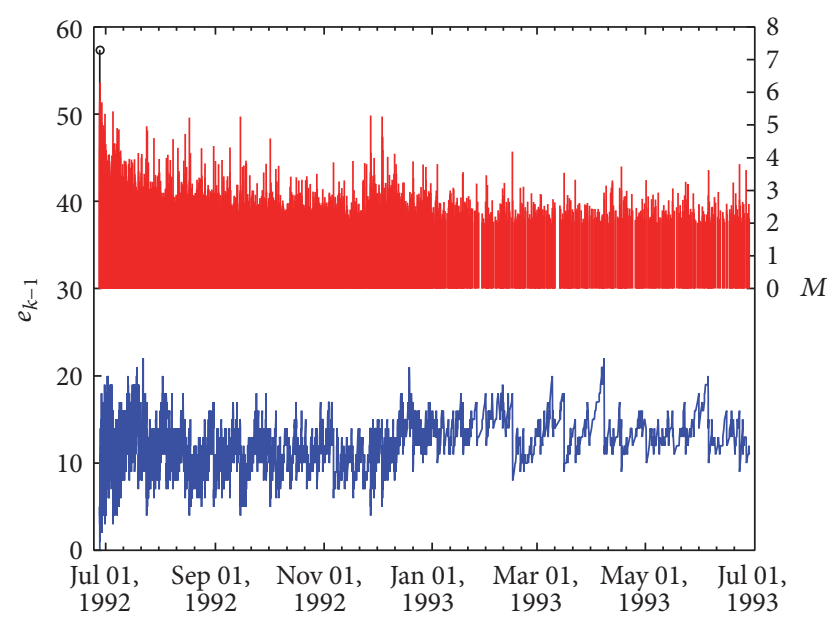

(a)

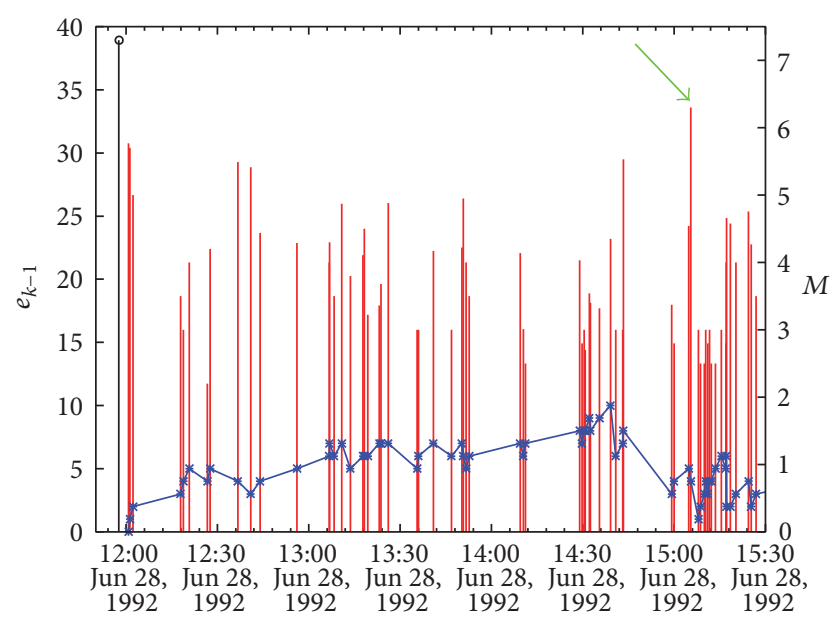

(b)

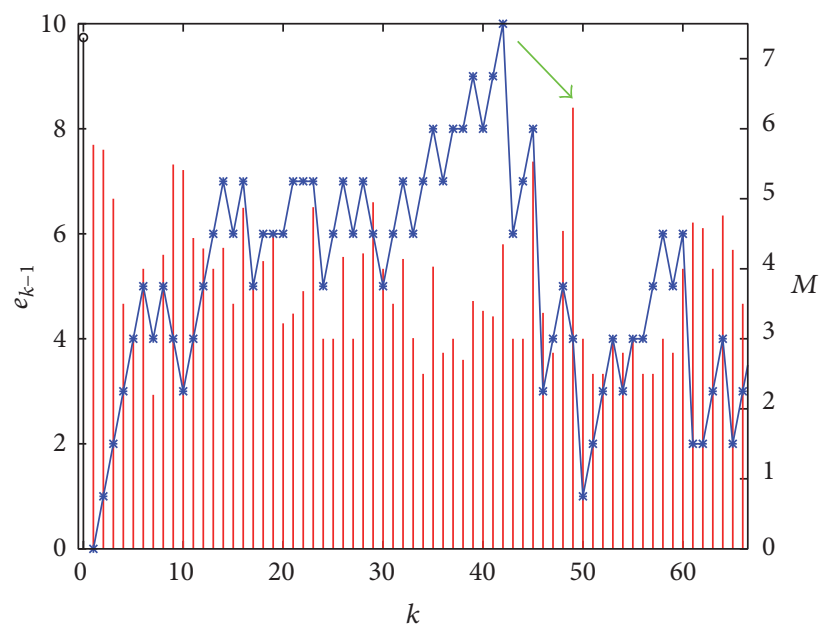

(c)

Figure 5: (a) The magnitude time series (right scale) of the Landers EQ (open circle) and its aftershocks (red vertical bars) together with the time series of $e_{k-1}$ (blue broken line, left scale). (b) is an excerpt of (a) for the first three and a half hours after the mainshock. (c) The results of (b) depicted in natural time. The green arrows in (b) and (c) indicate the Big Bear aftershock.

\section{Results and Discussion}

We first examine whether $e_{k}$ as it results from the aftershock time series exhibits $1 / f$ noise. To this end, we use the Detrended Fluctuation Analysis (DFA) [67-69] which is a standard robust method suitable for detecting long-range power-law correlations and has been applied to diverse fields where scale-invariant behavior emerges, for example, from DNA [70-72] and heart dynamics [68, 73] to meteorology $[74,75]$, atmospheric physics $[76,77]$, geophysics $[28,40,78$, 79], and economics [80-85]. The major advantage of DFA is the systematic elimination of polynomial trends of different order [86-89] (and hence of the much slower aforementioned logarithmic trend in $n_{k}$ ). In DFA, a fluctuation measure $F_{\text {DFA }}(l)$ is constructed, based on the residuals of a piecewise polynomial fitting to the profile time series, and studied versus the scale $l$ used for the polynomial detrending (e.g., for more details see Section 1.4 .3 of [7]). If $F_{\mathrm{DFA}}(l) \propto l^{\alpha}$ the time series exhibits power-law correlations and the power spectrum $S(f)\left(\propto 1 / f^{\beta}\right)$ exponent $\beta$ is related [70] to the DFA exponent $\alpha$ through $\beta=2 \alpha-1$. Figure 6 depicts the results of the DFA for the $e_{k}$ time series deduced from the aftershock magnitude time series of the four stronger EQs of Table 1. We observe that the resulting DFA exponents $\alpha$ are very close to unity, thus showing that $e_{k}$ indeed exhibits $1 / f$ noise.

We now turn to the prediction algorithm based on $e_{k}$ discussed in the previous section. This is applied in natural time. Thus, the time increased probability (TIP) $[90,91]$ is turned on after the occurrence of the $(k-1)$ th aftershock when $e_{k-1}$ is equal to or below some threshold $e_{t}$ and lasts until the occurrence of the (next) $k$ th aftershock with $M_{k} \geq$ $M_{\text {thres }}$. Based on Figure 1 of Shcherbakov et al. [13], we set $M_{\text {thres }}=2.0$. Under these assumptions, the aftershock magnitude prediction reduces to a binary prediction. We can therefore use the Receiver Operating Characteristics (ROC) graph [92] to depict the prediction quality. This is a plot of the 


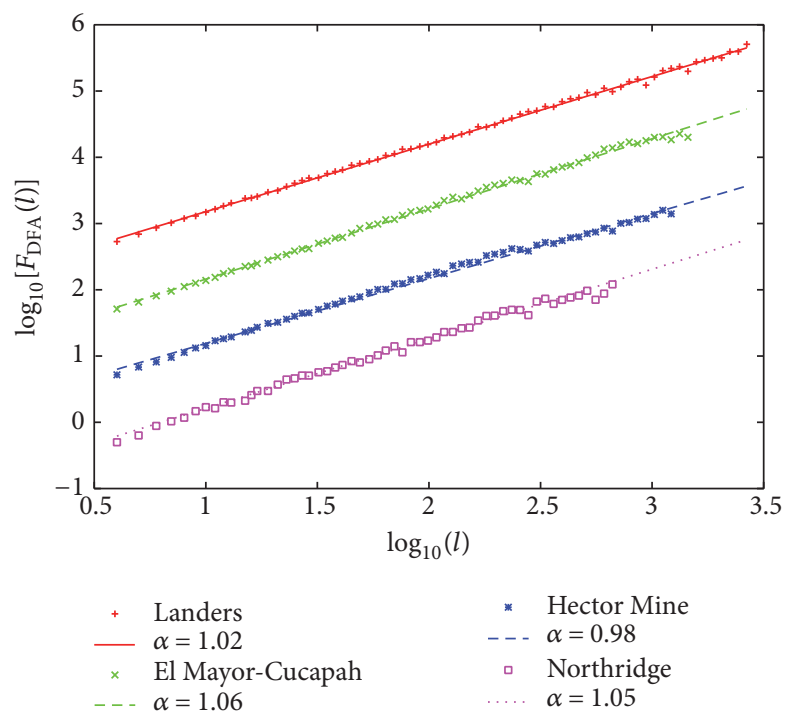

Figure 6: DFA results for the $e_{k}$ time series after the four stronger EQs (see Table 1) in Southern California during the period 1981 to June 2011. For reasons of clarity, $\log _{10}\left[\mathrm{~F}_{\mathrm{DFA}}(l)\right]$ have been vertically displaced by one unit starting from the bottom to the top. The least squares straight lines in each case are also shown together with the corresponding DFA exponent $\alpha$.

hit rate (or True Positive rate, TPr) versus the false alarm rate (or False Positive rate, FPr), as a function of the total rate of alarms, which is tuned by the threshold $e_{t}$. The hit rate is the ratio of the cases for which TIP was on and $M_{k} \geq M_{\text {target }}$ over the total number of cases that $M_{k} \geq M_{\text {target }}$. The false alarm rate is the ratio of the cases for which TIP was on and $M_{k}<$ $M_{\text {target }}$ over the total number of cases for which $M_{k}<M_{\text {target }}$. Random predictions generate equal hit and false alarm rate, and hence they lead to the diagonal in ROC plot. Thus, only when the points lie above this diagonal a predictor is useful.

Of course, random predictions lead to ROC curves that exhibit fluctuations which depend on the positive $P$ cases (when $M_{k} \geq M_{\text {target }}$ ) and the negative $Q$ cases (when $\left.M_{k}<M_{\text {target }}\right)$ to be predicted. The statistical significance of an ROC curve depends [93] on the area $A$ under the curve in the ROC plane (cf. this area is also usually termed AUC). Mason and Graham [93] have shown that

$$
A=1-\frac{U}{(P Q)},
$$

where $U$ follows the Mann-Whitney $U$-statistics [94]. Recently, a visualization scheme for the statistical significance of ROC curves has been proposed [95]. It is based on $k$ ellipses which are the envelopes of the confidence ellipses (cf. a point lies outside a confidence ellipse with probability $\exp (-k / 2)$ ) obtained when using a random predictor and vary the prediction threshold. These $k$-ellipses cover the whole ROC plane and using their $A$ we can have a measure [95] of the probability $p$ to obtain by chance (i.e., using a random predictor) an ROC curve passing through each point on the ROC plane. In the present case, the $p$ values that will be reported below correspond to the whole ROC curve and are calculated on the basis of its AUC using Eq. (2) by means of the computer program VISROC. $f$ available in Sarlis and Christopoulos [95]. This $p$ value gives the probability of obtaining by chance a prediction scheme that leads to an AUC equal to the observed one for given values of $P$ and $Q$. It is worthwhile to mention that in the standard method, by using the Gutenberg-Richter law which is a skewed distribution for which ROC graphs are [92] especially useful, the magnitudes are assumed to be independent, thus unpredictable, and this remains so even if we consider the so-called [96] shortterm aftershock incompleteness (STAI) which mainly arises because directly after a large EQ the overlapping arrivals of waves from different events can be masked [97-99] thus temporarily increasing the completeness threshold of the EQ catalogue (see also the discussion below). On the other hand, the present method tries to predict large aftershocks from the correlation between magnitudes. Below in this paper, we focus on evaluating the statistical significance of this method. Other methods may be also needed to evaluate its practical utility.

3.1. The Case of the Four Stronger EQs $(M \geq 6.7)$ in Southern California. These are the cases of the 1992 Landers, the 2010 El Mayor-Cucapah, the 1999 Hector Mine, and the 1994 Northridge EQ. As mentioned the analysis is made by considering the areas shown in Table 1 centered at the epicenter of each mainshock and for a period $T$ equal to one year. The aftershocks thus obtained (see Figure 1) have been sorted according to their origin time and the time series $M_{k}$ versus the order of occurrence $k$ has been constructed. Figure 5 shows an example of the time series $e_{k}$ obtained from the successive extrema of the aftershock magnitude time series $M_{k}$ of the 1992 Landers EQ. We then apply the prediction algorithm as described in the previous section for various target magnitudes $M_{\text {target }}$ : when $e_{k-1}$ is smaller than or equal to $e_{t}$ the TIP turns on: if the magnitude of the (next) aftershock is $M_{k} \geq M_{\text {target }}$ we have a true positive successful prediction and if $M_{k}<M_{\text {target }}$ we have a false positive unsuccessful prediction. When TIP is off, that is, $e_{k-1}>e_{t}$, and 

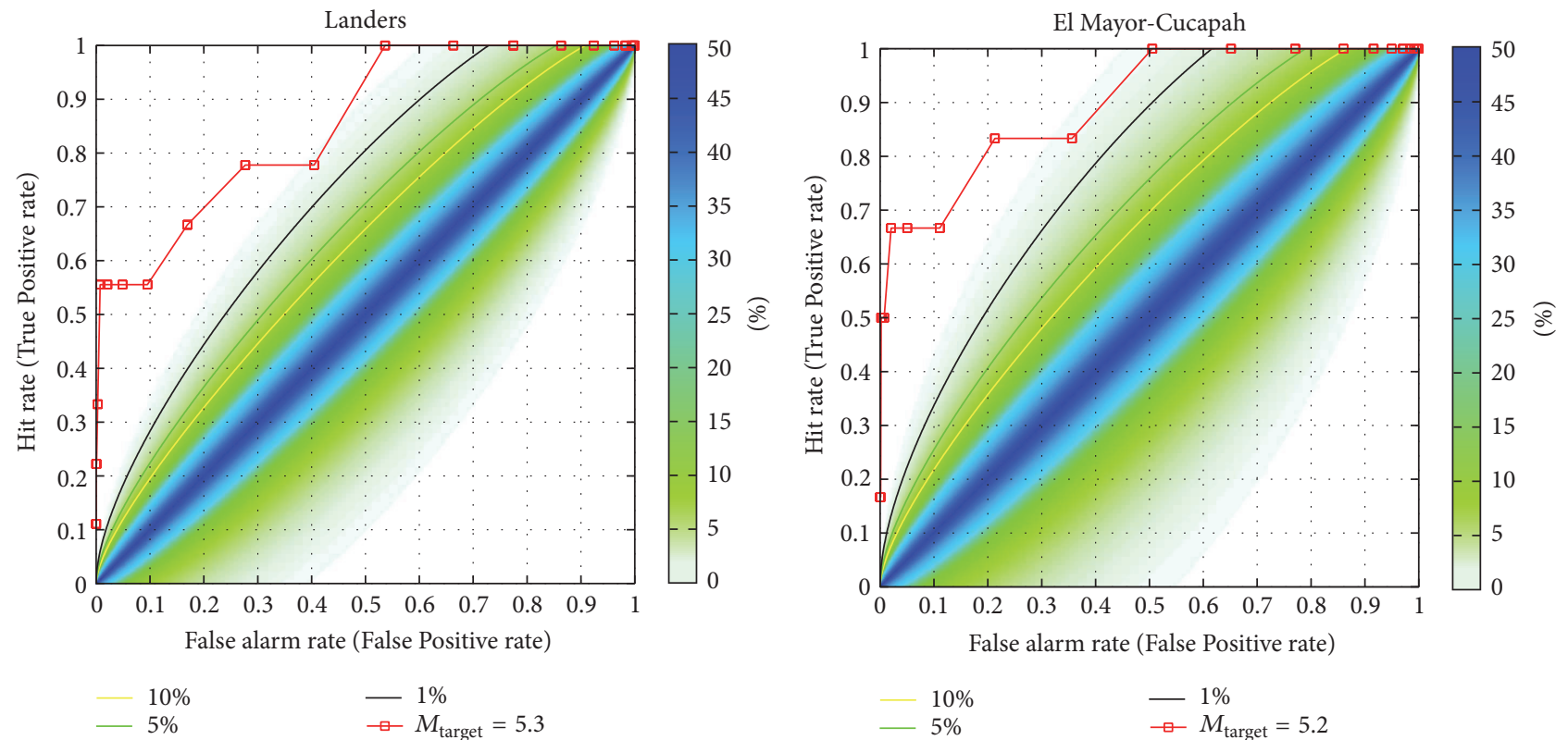

(a)

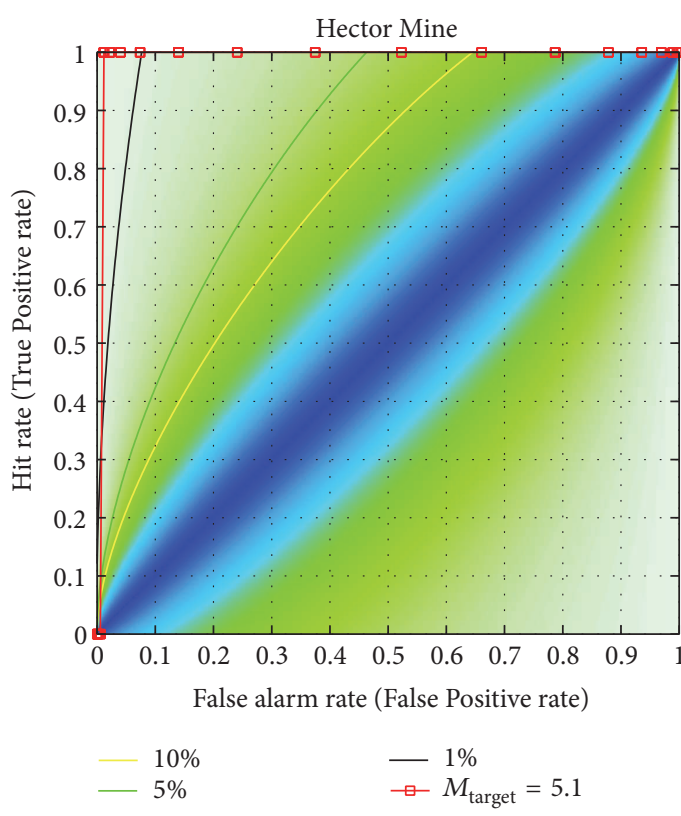

(c)
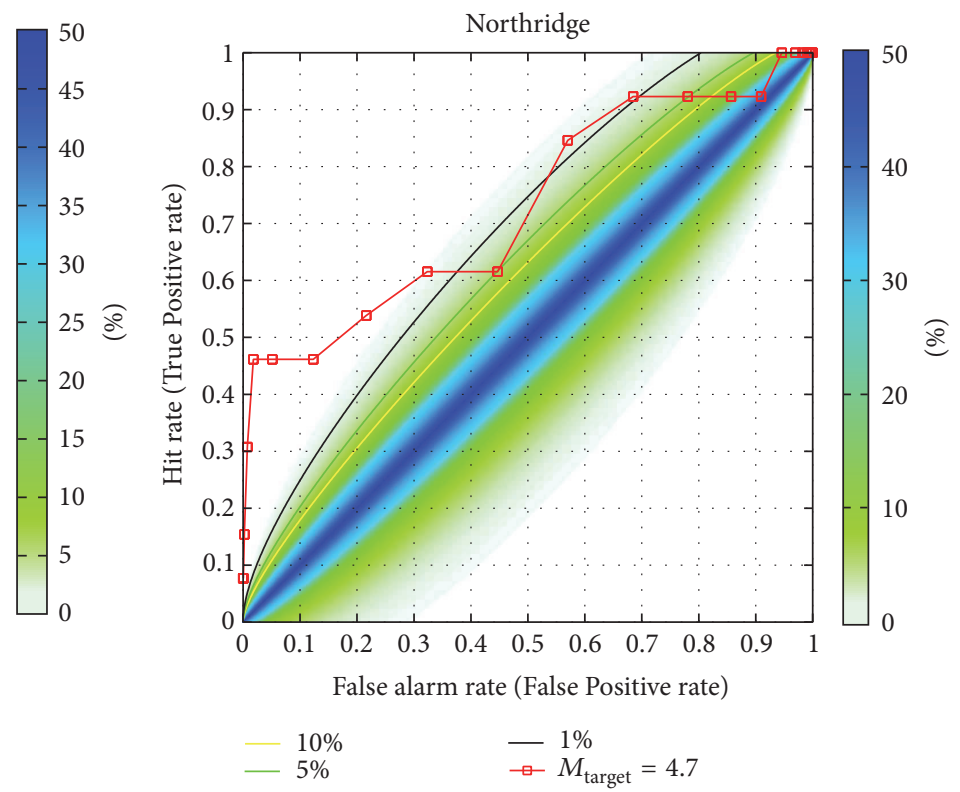

(d)

Figure 7: Receiver Operating Characteristics (red squares) when using $e_{k}$ as a predictor for $M_{\text {target }}=M_{m}-2$ for the aftershock time series of Landers, El Mayor-Cucapah, Hector Mine, and Northridge EQs (see Table 1). In each case, the colored contours present the $p$ value to obtain by chance an ROC curve based on $k$-ellipses [95]; the $k$-ellipses with $p=10 \%, 5 \%$, and $1 \%$ are also shown.

$M_{k}<M_{\text {target }}$ we have a true negative successful prediction; if $M_{k} \geq M_{\text {target }}$ we have false negative unsuccessful prediction, a miss. This way we can obtain ROC curves for various $M_{\text {target }}$.

We first focus on a high $M_{\text {target }}$, selected on the first decimal digit, that is determined by the formula $M_{\text {target }}=M_{m}-$ 2, where $M_{m}$ is-as mentioned-the mainshock magnitude. The results for these $M_{\text {target }}$ 's for the four strong EQs under discussion are shown in Figure 7. The corresponding $p$ values to obtain such ROC curves by chance is $0.012 \%, 0.036 \%$, $0.81 \%$, and $0.24 \%$ for the Landers, El Mayor-Cucapah, Hector
Mine, and Northridge EQ, respectively. Figure 8 depicts the ROC curves obtained when selecting a lower target magnitude $M_{\text {target }}=4.0$. We observe that in this case also we obtain ROC curves of high statistical significance. Finally, in Figures 9(a) and 9(b), we present the results obtained for AUC and the corresponding $p$ values when considering a wide range of $M_{\text {target }}$ 's from 4.0 to 5.5 .

3.2. The Case of the Four Smaller EQs $(6.6 \geq M \geq 6.0)$. As already mentioned, this is the case of the two Superstition 


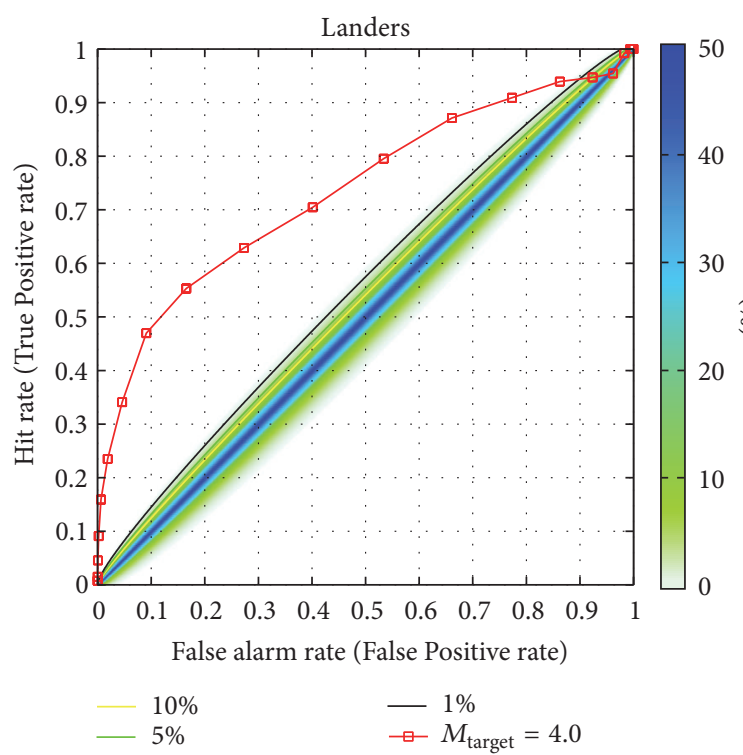

(a)

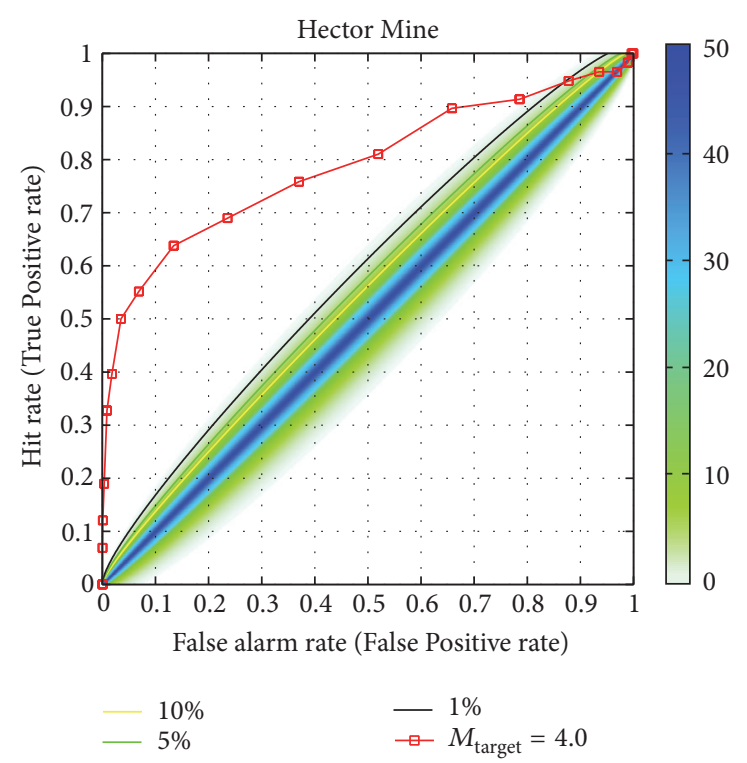

(c)

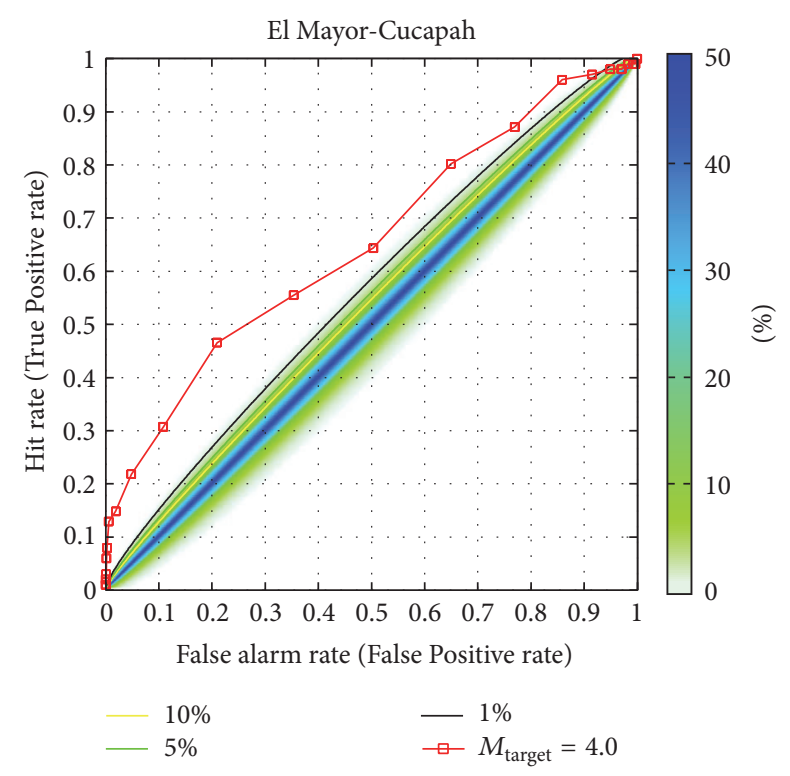

(b)

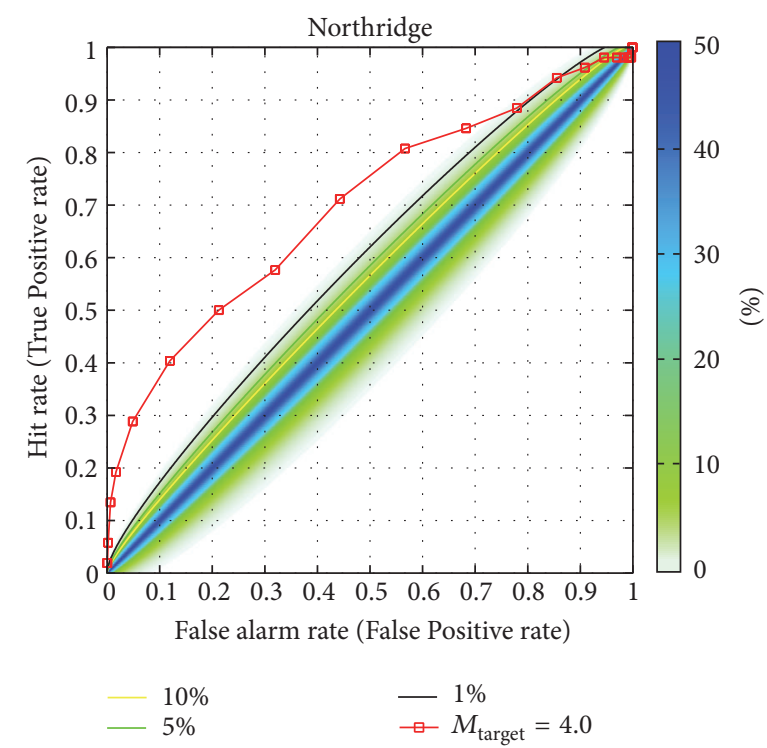

(d)

Figure 8: Receiver Operating Characteristics (red squares) when using $e_{k}$ as a predictor for $M_{\text {target }}=4.0$ for the aftershock time series of Landers, El Mayor-Cucapah, Hector Mine, and Northridge EQs, respectively. In each case, the colored contours present the $p$ value to obtain by chance an ROC curve based on $k$-ellipses [95]; the $k$-ellipses with $p=10 \%, 5 \%$, and $1 \%$ are also shown.

Hills large events [53] in 1987 and the 1992 Joshua Tree and Big Bear events which are related $[54,55]$ to the Landers EQ. The areas used for the selection of the aftershock magnitude time series $M_{k}$ are shown in the last column of Table 1, but now the period $T$ for the study of aftershocks may vary from one year since these EQs cannot be considered as clearly independent mainshocks. Specifically, in the case of the first Superstition Hills M6.2 EQ, we considered as $T$ the period until the occurrence of the second Superstition Hills M6.6 EQ, that is, roughly 11 hours and 23 minutes. Moreover, since Joshua Tree EQ is clearly related $[54,55]$ to the Landers EQ, we considered as $T$ the period until the occurrence time of the Landers EQ, that is, roughly 66 days and 7 hours. In the remaining two cases treated here, a period $T$ of one year has been used. The aftershocks considered in each case are shown in Figure 2.

Following the procedure used in the previous subsection, we sorted the aftershocks according to their occurrence time and constructed the $M_{k}$ time series, and from the latter we obtained the predictor time series $e_{k}$. This time series has been used for the prediction of the aftershock magnitude time series $M_{k}$ and the ROC curves obtained are shown in Figures 10 and 11 for $M_{\text {target }}=M_{m}-2$ and $M_{\text {target }}=3.5$, respectively, for each EQ. On the latter figure, we clearly observe that the predictions made on the basis of the proposed algorithm are 


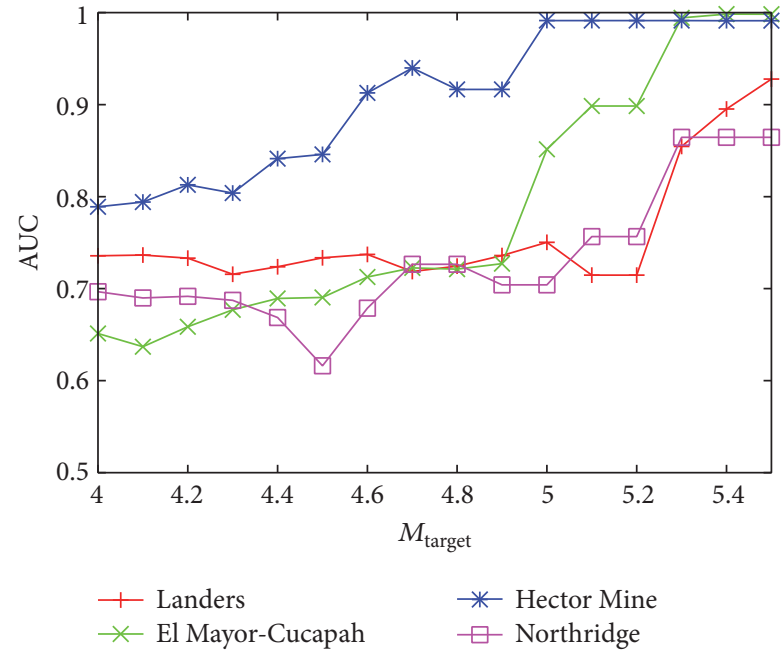

(a)

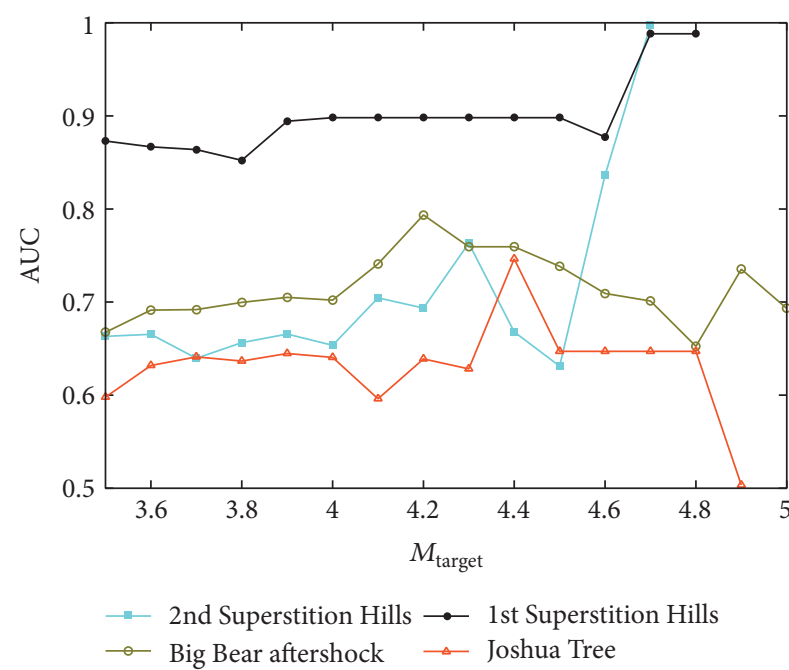

(c)

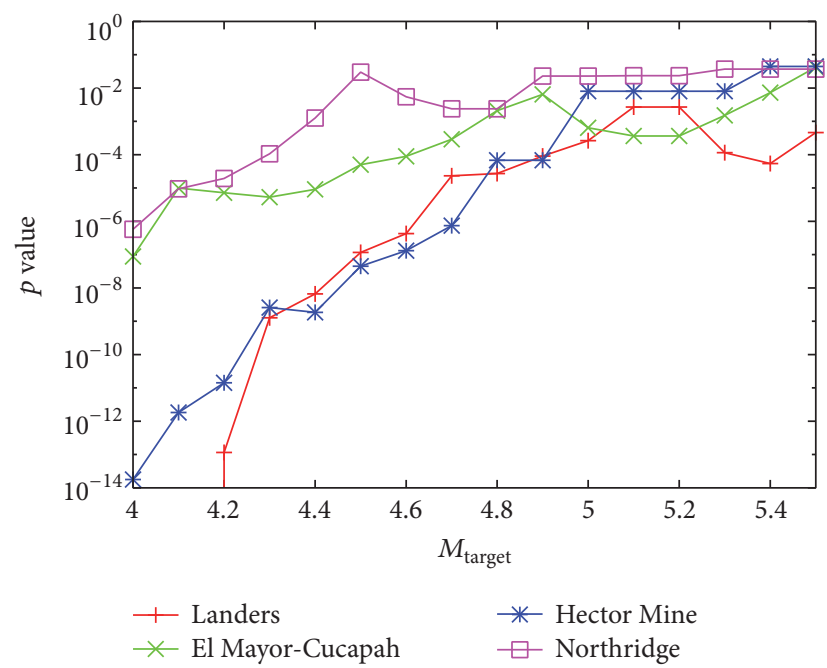

(b)

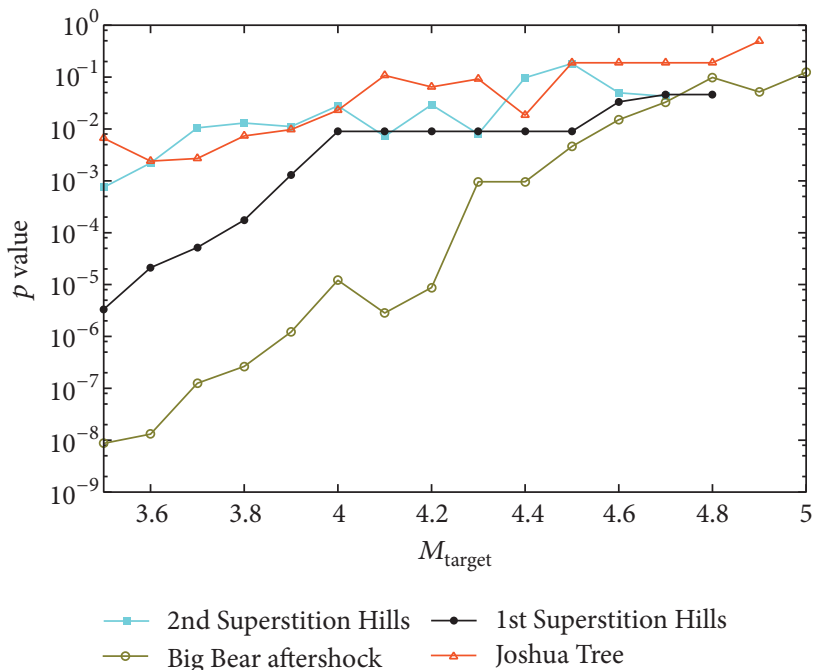

(d)

FIGURE 9: Results obtained from the analysis of the four stronger ((a), (b)) and the four smaller ((c), (d)) EQs in Southern California by ROC diagrams. Panels (a) and (c) depict AUC versus $M_{\text {target }}$ while (b) and (d) depict the corresponding $p$ values.

far beyond chance (cf. for the ROC related to the Joshua Tree EQ in Figure 11 we have $p=0.7 \%$ ). If we focus our attention on the high $M_{\text {thres }}$ 's (see Figure 10), larger $p$ values are obtained which are $5 \%, 0.1 \%, 0.9 \%$, and $11 \%$ for the second Superstition Hills, Big Bear, first Superstition Hills, and Joshua Tree EQs, respectively. These $p$ values are also larger than those obtained in the previous subsection when studying the stronger EQs in Southern California. In Figures 9(c) and 9 (d), we present the results obtained for AUC and the corresponding $p$ values when considering a wide range of $M_{\text {target }}$ 's from 3.5 to 5.0. Figure 9 (d) shows that in the vast majority (45 out) of the 59 examined cases the obtained $p$ values are below $5 \%$ pointing to the statistical significance of the method.

At this point we have to comment on the values of $e_{k}$ obtained after the last aftershock considered in the case of the first Superstition Hills and the 1992 Joshua Tree EQs. As mentioned our study above was terminated just before the occurrence of the second Superstition Hills and the Landers EQ, respectively, in each case. Hence, these values coincide with the $e_{k-1}$ values just before the occurrence of the latter two EQs and are 11 and 7, respectively. The operating points on the ROC diagram corresponding to $e_{t}$ equal to these values are shown with the red arrows in Figure 10. An inspection of this figure shows that depending of the selection of the operating point of the proposed algorithm an alarm could have been probably on almost 23 hours before the occurrence of the Landers EQ (actually after 12:38:20 UTC on June 27, 1992 , when an $M=2.36$ EQ occurred at $34.046 \mathrm{~N} 116.287 \mathrm{~W}$, i.e., $22 \mathrm{~km}$ away from the Landers EQ epicenter) whereas this is rather improbable for the case of the second Superstition Hills EQ since the corresponding to $e_{t}=11$ ROC point leads to an extremely high false alarm rate. Hence, the selection 


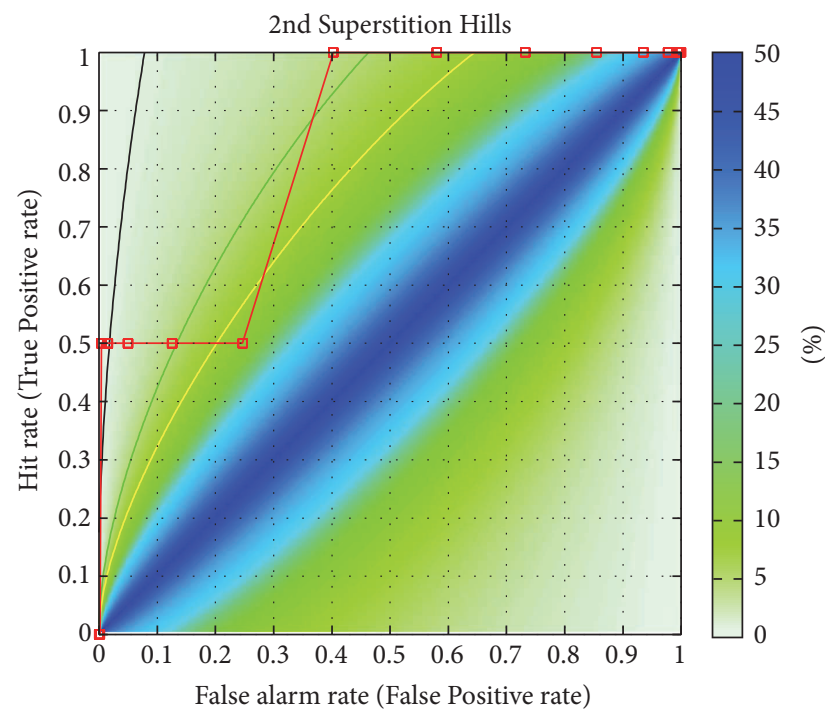

$\begin{array}{ll}-10 \% & -1 \% \\ -5 \% & -M_{\text {target }}=4.6\end{array}$

(a)

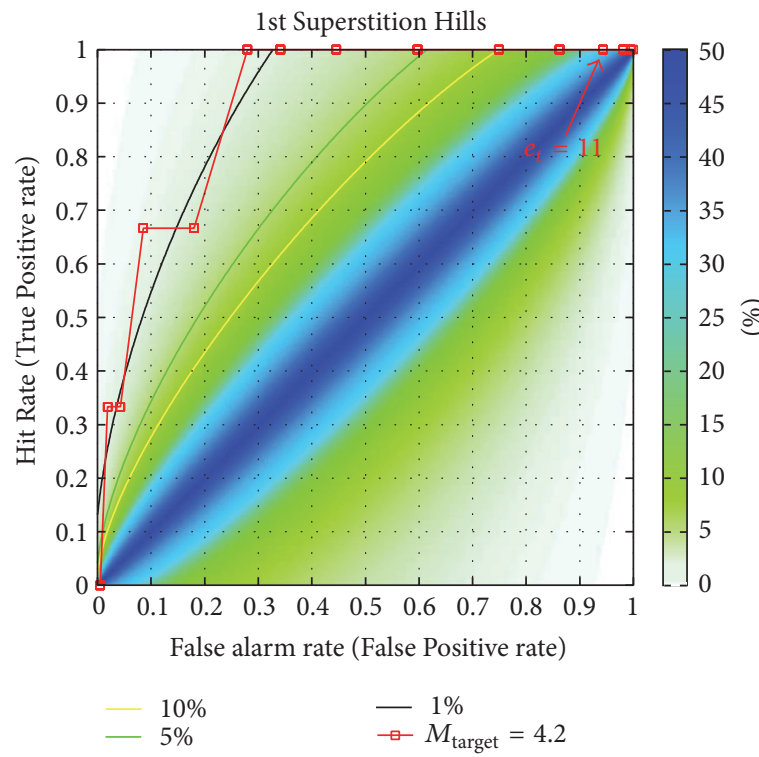

(c)

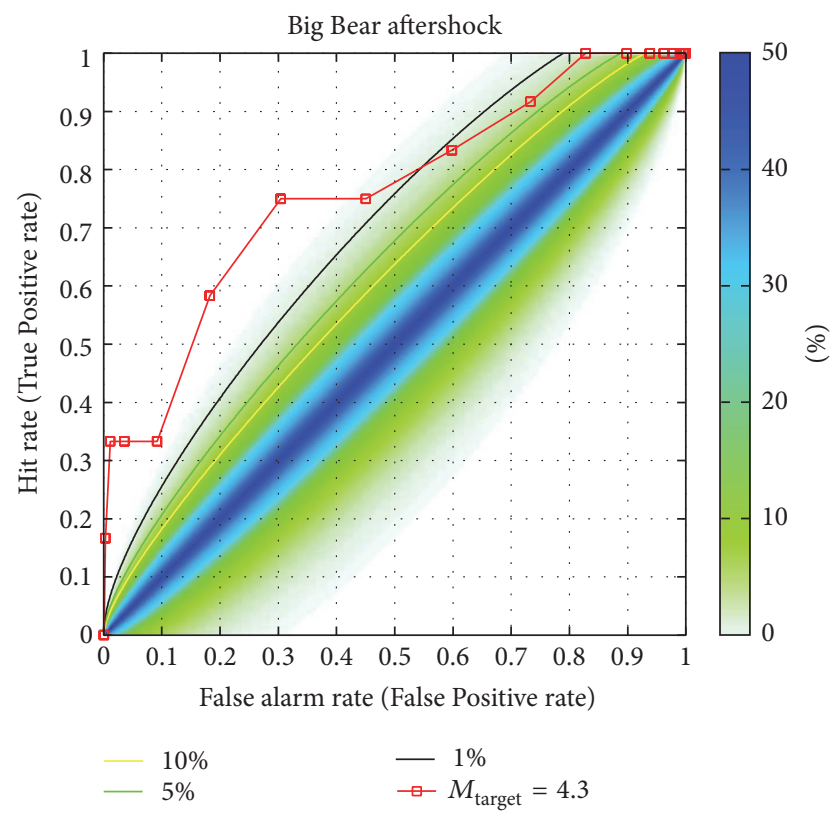

(b)

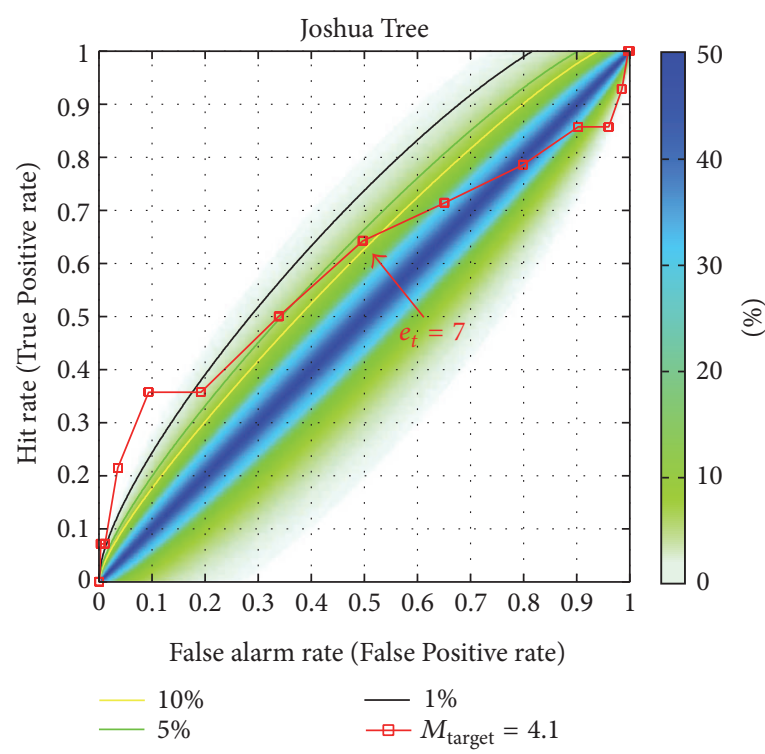

(d)

FIGURE 10: Receiver Operating Characteristics (red squares) when using $e_{k}$ as a predictor for $M_{\text {target }}=M_{m}-2$ for the aftershock time series of the second Superstition Hills, the Big Bear aftershock, the first Superstition Hills, and the Joshua Tree EQs (see Table 1). In each case, the colored contours present the $p$ value to obtain by chance an ROC curve based on $k$-ellipses [95]; the $k$-ellipses with $p=10 \%, 5 \%$, and $1 \%$ are also shown. The red arrows indicate the points on the ROC diagram that correspond to $e_{t}=11$ and $e_{t}=7$ that coincide with the values of $e_{k-1}$ just before the occurrence of the second Superstition Hills and the Landers EQ obtained from the analysis of the aftershocks of the first Superstition Hills and Joshua Tree EQs, respectively.

of $e_{t}$ is also very important. The latter point together with its implications for a practical application of the proposed algorithm will be discussed in the next subsection.

3.3. Further Analysis of the Results. We first focus on the fact that the prediction algorithm presented here is statistically significant. This statistical significance is very high when considering a relatively small $M_{\text {target }}$ (e.g., see Figures 8 and
11), which also pertains when focusing on $M_{\text {target }}=M_{m}-2$ (e.g., see Figures 7 and 10). A comparison of Figures 9(b) and 9 (d) shows that this statistical significance may decrease when we consider the smallest in magnitude EQ, that is, the Joshua Tree EQ. Even in this case, however, we obtain $p$ values which exclude the possibility of a random result.

In order to evaluate the statistical significance of the proposed algorithm out of sample and for EQs in regions 


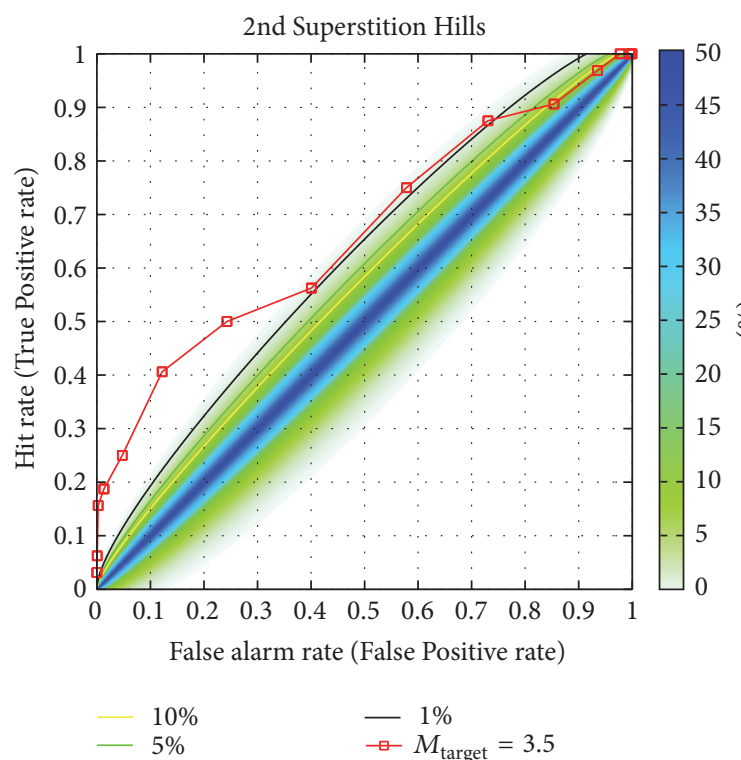

(a)

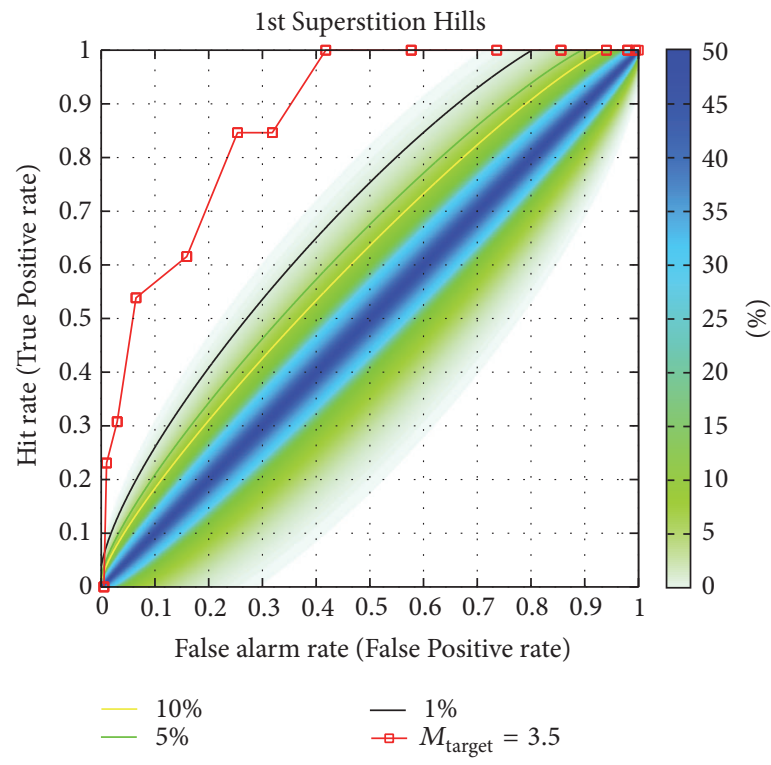

(c)

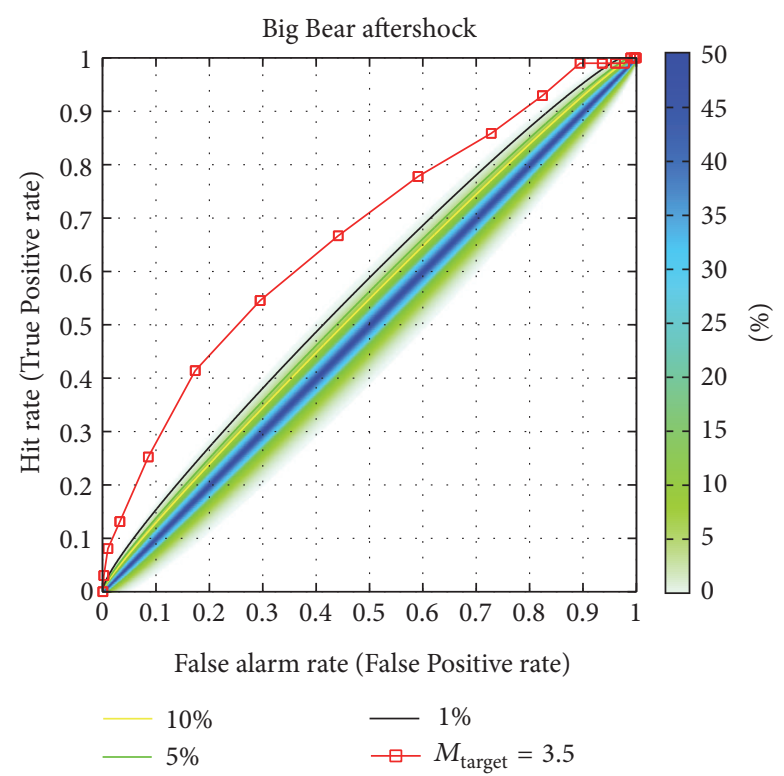

(b)

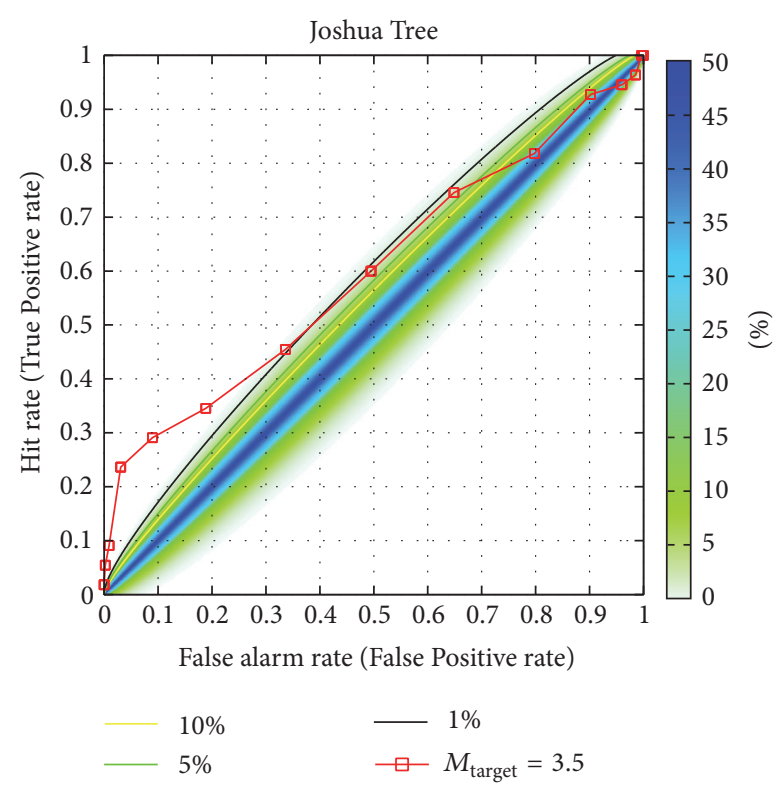

(d)

FIGURE 11: Receiver Operating Characteristics (red squares) when using $e_{k}$ as a predictor for $M_{\text {target }}=3.5$ for the aftershock time series of the second Superstition Hills, the Big Bear aftershock, the first Superstition Hills, and the Joshua Tree EQs, respectively. In each case, the colored contours present the $p$ value to obtain by chance an ROC curve based on $k$-ellipses [95]; the $k$-ellipses with $p=10 \%, 5 \%$, and $1 \%$ are also shown.

different from California, we proceeded, by employing the same procedure as that described in Materials and Methods, to the analysis of all $M \geq 8.5 \mathrm{EQs}$ in the Earth since 1976 (see Table 2). For this study the data analyzed come from the Global Centroid Moment Tensor (CMT) Project [100, 101] that covers global seismicity since 1 January 1976 and we considered $M_{\text {thres }}=5.0$, as in Sarlis et al. [51]. For EQs that took place before 2011, the 1976 to 2010 CMT catalogue was used, whereas for EQs since 1 January 2011 to 1 July 2015 the monthly CMT catalogues have been employed (all these catalogues are publicly available from http://www.globalcmt.org/CMTfiles .html). For reasons of brevity, we will refer to these data as the GCMT catalogue. The AUC and $p$ values obtained for the wide range of $M_{\text {target }}$ from 6.0 to 7.5 are depicted in Figure 12 in a fashion similar to that of Figure 9. From the six cases studied, only the results obtained for the 2005 Sumatra-Nias EQ do not appear statistically significant. This fact has been also verified by producing $10^{2}$ randomly shuffled copies of the original time series and comparing whether the AUCs obtained for a shuffled copy time series were higher 


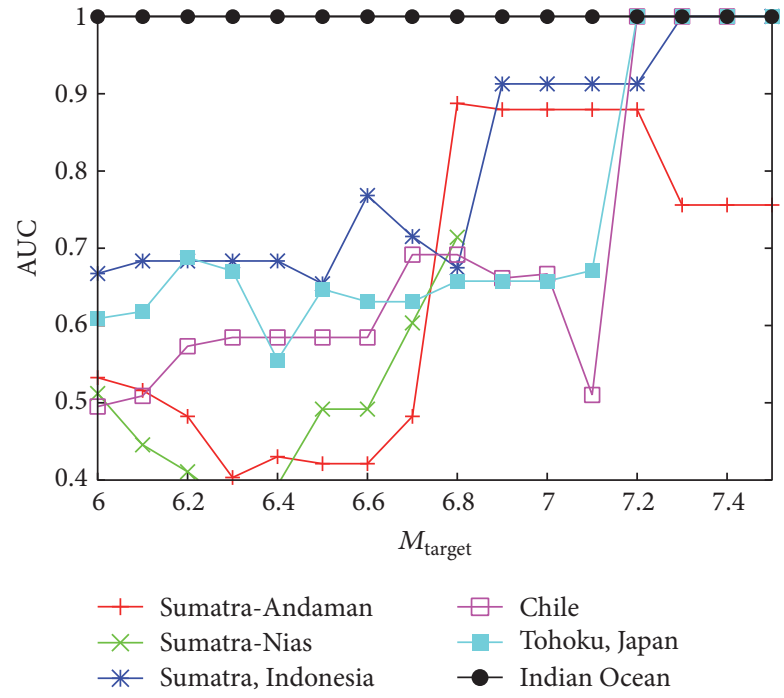

(a)

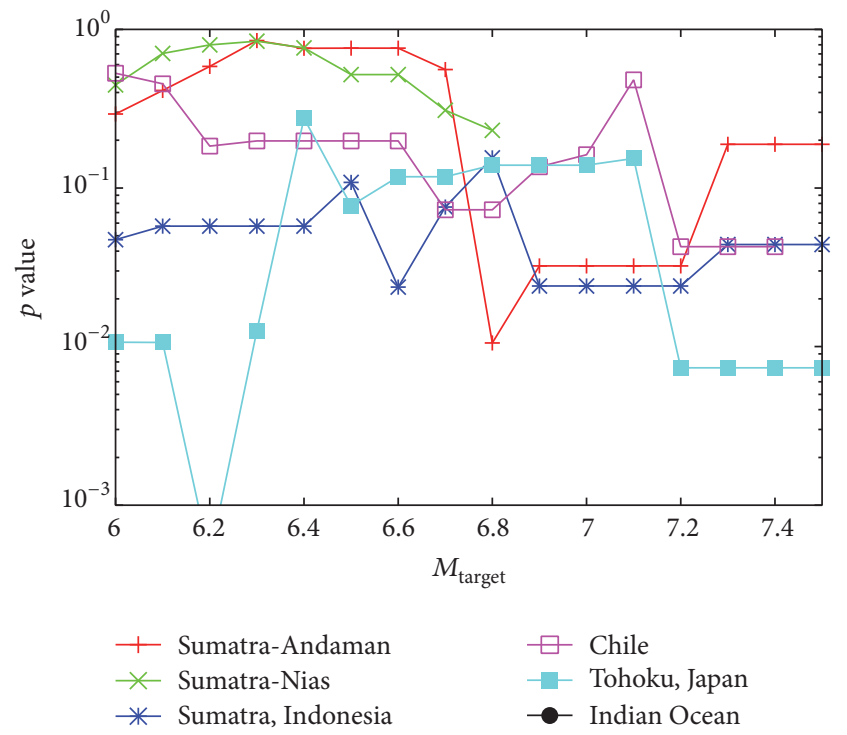

(b)

Figure 12: Results obtained for all $M \geq 8.5$ EQs according to the GCMT catalogue (see Table 2) by ROC diagrams. Panels (a) and (b) depict the AUC and the corresponding $p$ values, respectively.

than those depicted in the majority of cases presented in Figure 12(a) for each EQ: For the 2005 Sumatra-Nias EQ in $54 \%$ of the cases the AUCs for the shuffled copies were higher than those depicted in Figure 12(a), while this percentage falls to $9 \%$ and $8 \%$ for the Sumatra-Andaman and Chile cases. For the other three cases studied the obtained percentages were smaller than $2 \%$. We note, however, that the 2005 SumatraNias EQ occurred almost three months after the SumatraAndaman EQ and is included within the rectangular region of the latter EQ (see Table 2). The corresponding $e_{k-1}$ value (almost three and a half days) before the 2005 Sumatra-Nias EQ was 4, which is the same as that before the Big Bear aftershock; see Figure 5(c).

An inspection of Figure 12(b) also shows that the $p$ values are much larger than those of Figure 9. This is related to the fact that $M_{\text {thres }}$ used for GCMT is much larger than that used in Southern California where an accurate waveform relocated regional catalogue $[8,9]$ has been employed. Using a higher $M_{\text {thres }}$ does not allow $e_{k}$ to vary significantly since there are no small EQs to increase $e_{k}$. For example, the maximum value of $e_{k}$ is $10,9,10,11,17$, and 6 for the six EQs of GCMT presented in the first column of Table 2 , respectively, compared to 22,22 , $22,24,15,19,14$, and 15 for the EQs presented in the first column of Table 1 in Southern California. Hence, the threshold $e_{t}$ has to be modified accordingly. Figure 13 depicts two selections of operating points, corresponding to fixed $e_{t}$ for each catalogue, for the totality of the 14 EQs studied when focusing on the prediction of aftershocks with $M \geq M_{m}-2$ and could be seen as a summary evaluation for various regions and two different catalogues (we will return to this point later).

Let us now return to the cases in Southern California and discuss the effect of STAI on the statistical significance of the method. In order to correct from this effect, we followed Helmstetter et al. [102, 103], considered for each EQ of Table 1 a time varying $M_{\text {thres }}$ given by

$$
M_{\text {thres }}\left(M_{m}, t\right)=\max \left[M_{m}-4.5-0.75 \log _{10}(t), 2.0\right] \text {, }
$$

where $t$ is the elapsed time measured in days from the mainshock, that is, Eq. (15) of Helmstetter et al. [103], and repeated the calculations focusing only on the aftershocks during the first 24 hours after each mainshock where STAI is stronger. The results are shown in Figure 14 and show that out of the 115 obtained $p$ values only 6 exceed $7 \%$ whereas the vast majority, that is, 107 cases, are below 5\%.

As an additional check, we examined whether the validity of the proposed method originates from STAI by the following test: each time the magnitude $M_{k}$ of a reported aftershock satisfied $M_{k} \geq M_{\text {thres }}\left(M_{m}, t\right), M_{k}$ was considered as the new element of a time series labeled $M_{l}^{\text {orig }}$ (original). At the same time, a magnitude $M_{k^{\prime}}\left[\geq M_{\text {thres }}\left(M_{m}, t\right)\right]$ has been randomly selected from the original distribution of $M_{k}$ and considered as the new element of a time series labeled $M_{l}^{\text {synth }}$ (synthetic). The two time series $M_{l}^{\text {orig }}$ and $M_{l}^{\text {synth }}$ have been analyzed by ROC using the prediction algorithm and the corresponding AUCs have been compared $10^{4}$ times. The results showed that the percentage of cases for which the AUC of a synthetic time series was larger in the majority of cases presented in Figure 9 than that of the original one is $4.2 \%$, $8.2 \%, 0.6 \%, 9.9 \%, 59 \%, 0.005 \%, 7.2 \%$, and $9.5 \%$ for the $8 \mathrm{EQs}$ presented in the first column of Table 1, respectively. These results (apart from those concerning the second Superstition Hills M6.6 EQ that will be discussed below) indicate that the observed predictability cannot be fully accounted for by STAI (cf. if that was the case, only up to two of the above 8 numbers 
TABLE 2: All EQs with magnitude $M \geq 8.5$ during the period 1 January 1976 to 1 July 2015 reported in the GCMT catalogue. The dimensions of the rectangular aftershock area, centered at each epicenter, used here are also shown.

\begin{tabular}{|c|c|c|c|c|c|}
\hline EQ name & $M$ & Origin time (UTC) & Lat $\left(^{\circ}\right)$ & Lon $\left(^{\circ}\right)$ & Area considered \\
\hline Sumatra-Andaman & 9.0 & $2004 / 12 / 2600: 58: 50$ & 3.30 & 95.78 & $7.8^{\circ} \times 7.8^{\circ}$ \\
\hline Sumatra-Nias & 8.6 & 2005/03/28 16:09:36 & 2.09 & 97.11 & $4.9^{\circ} \times 4.9^{\circ}$ \\
\hline Sumatra, Indonesia & 8.5 & 2007/09/12 11:10:26 & -4.44 & 101.37 & $4.4^{\circ} \times 4.4^{\circ}$ \\
\hline Chile & 8.8 & 2010/02/27 06:34:15 & -35.85 & -72.71 & $6.2^{\circ} \times 6.2^{\circ}$ \\
\hline Tohoku, Japan & 9.1 & 2011/03/11 05:46:23 & 38.32 & 142.37 & $8.7^{\circ} \times 8.7^{\circ}$ \\
\hline Indian Ocean & 8.6 & 2012/04/11 08:38:36 & 2.33 & 93.06 & $4.9^{\circ} \times 4.9^{\circ}$ \\
\hline
\end{tabular}

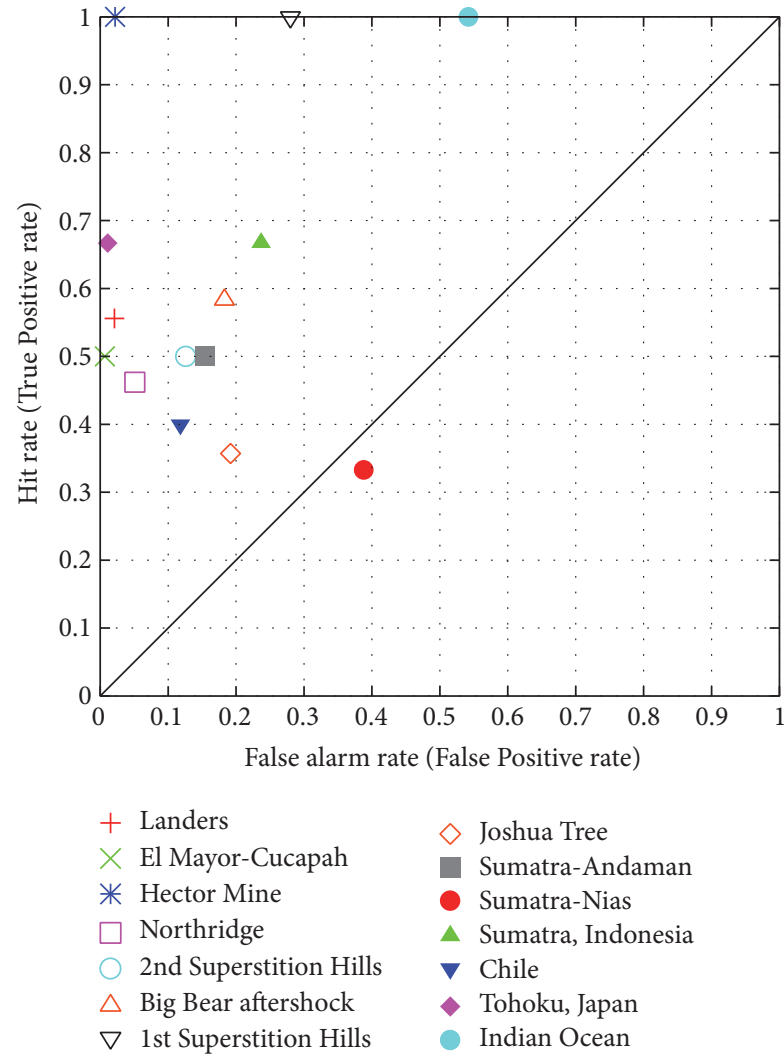

(a)

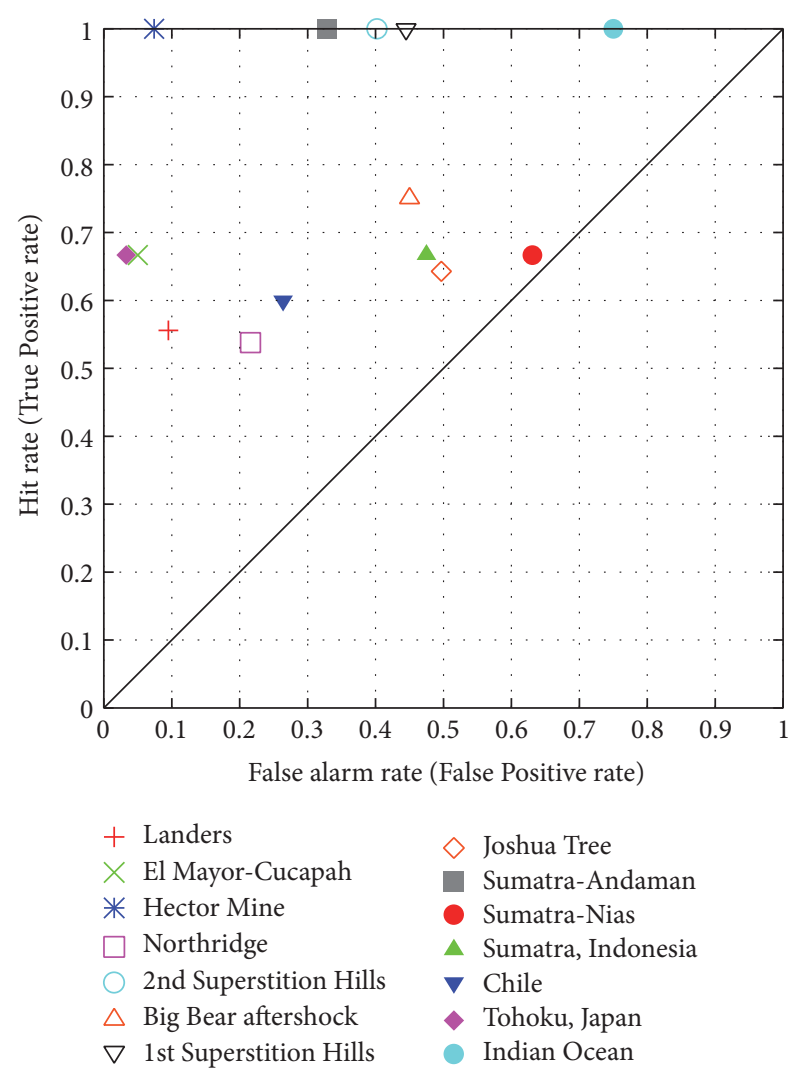

(b)

FIGURE 13: Operating points on the ROC diagram when using $e_{k}$ as a predictor for $M_{\text {target }}=M_{m}-2$ for all mainshocks studied (see Tables 1 and 2) (a) when adopting for Southern California (SC) $e_{t}{ }^{\mathrm{SC}}=5$ and for GCMT $e_{t}{ }^{\mathrm{GCMT}}=3$. Panel (b) corresponds to the selection $e_{t}{ }^{\mathrm{SC}}=7$ and $e_{t}^{\mathrm{GCMT}}=4$.

should have been below $10 \%$ with probability $96.2 \%\left(\approx 0.9^{8}+\right.$ $\left.8 \cdot 0.1 \cdot 0.9^{7}+4 \cdot 7 \cdot 0.1^{2} \cdot 0.9^{6}\right)$ due to the binomial distribution).

One might also argue that the present results could be alternatively reproduced by synthetic models generated using the Epidemic Type Aftershock Sequence (ETAS) model [105], coupling the main statistical laws describing the occurrence of earthquakes in time, space in magnitude, that is, the Gutenberg-Richter magnitude distribution and the OmoriUtsu law as well as the increase of the number of aftershocks as about $10^{M_{m}}$ with the mainshock magnitude $M_{m}$ (or Bath law). Following Zhuang and Touati [106], we used the code etasim.f of SASeis2006 [104]; see also [105, 107, 108], for
ETAS. By simulating magnitude by Gutenberg-Richter law for $b=1$ with cutoff magnitude 2.0 , we compared the results thus obtained (blue asterisks in Figure 15) with those of the test data provided in SASeis2006 (http://www.ism.ac.jp/ $\sim$ ogata/Ssg/softwares.html). These test data come [104] from the Japan Meteorogical Agency (JMA) EQ catalogue and concern the aftershock data for almost 18 days of the 26 July 2003 EQ of $M_{\mathrm{JMA}}=6.2$ at the northern Miyagi-Ken, northern Japan, and the aftershock data for almost 6 days of the 16 August 2005 EQ of $M_{\text {JMA }}=7.2$ offshore of western FukuokaKen, Kyushu, Japan. Both the real test data and the simulating $b=1$ time series have been considered with $M_{\text {thres }}=2.0$ and 


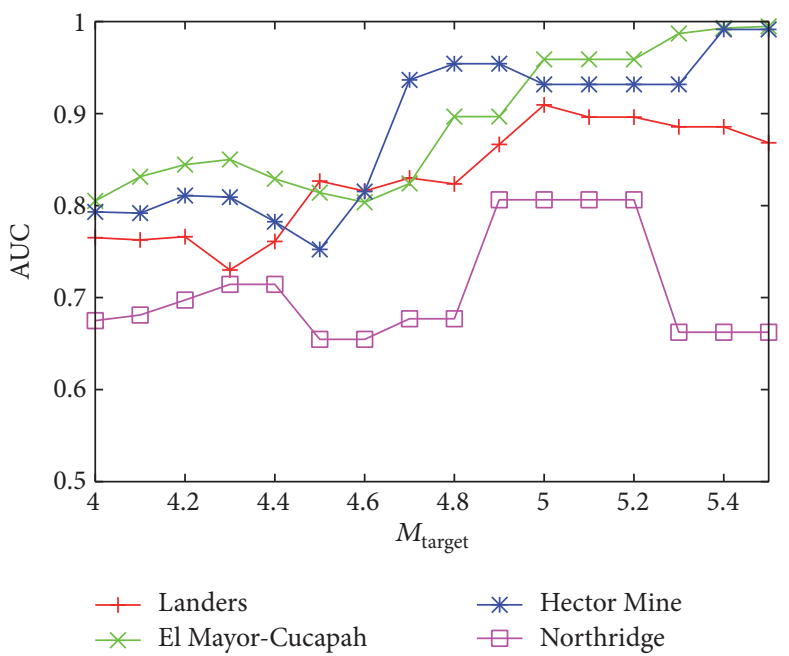

(a)

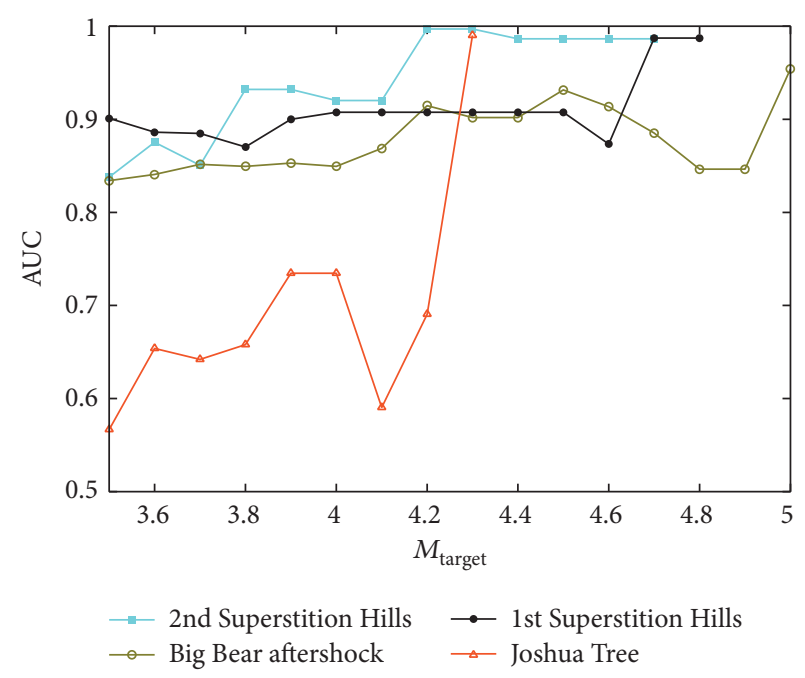

(c)

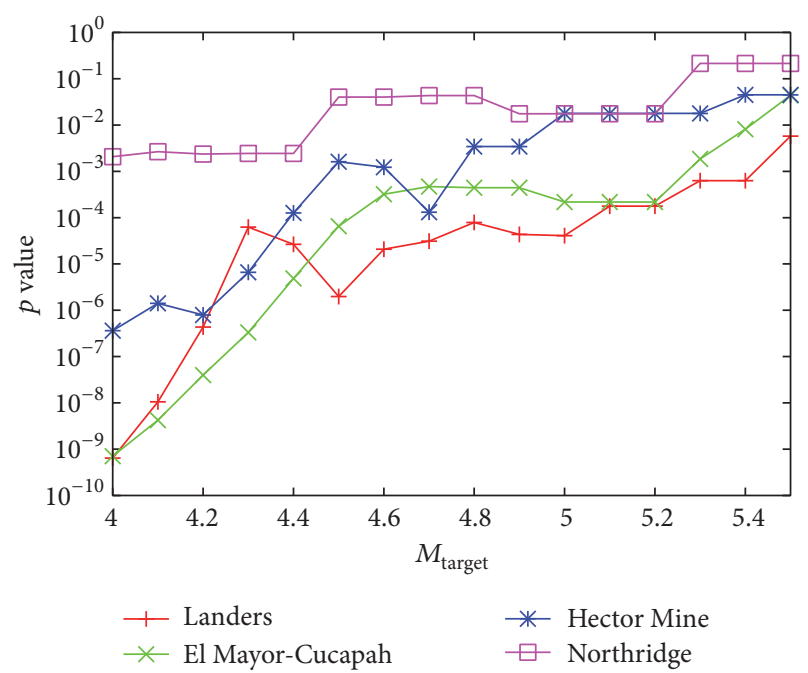

(b)

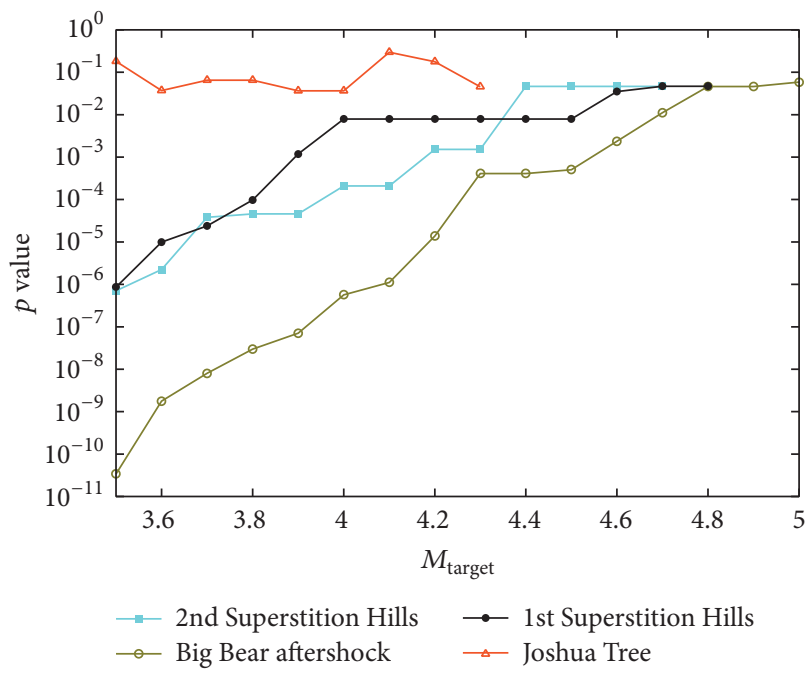

(d)

FIGURE 14: Results obtained when focusing on the first 24 hours after the mainshock with time variable threshold $M_{\text {thres }}\left(M_{m}, t\right)$ from the analysis of the four stronger ((a), (b)) and the four smaller ((c), (d)) EQs by ROC diagrams. Panels (a) and (c) depict AUC versus $M_{\text {target }}$ while (b) and (d) depict the corresponding $p$ values.

the algorithm has been applied by using the code presented in Appendix. Figure 15 depicts the AUCs and the corresponding $p$ values. An inspection of this figure shows that the results obtained for the real data clearly outperform the simulated ones since in only 3 cases (out of 48) the AUC of the ETAS simulated data exceeded that of the real data. Thus, the experimentally observed predictability cannot be reproduced. These results also show that the present algorithm conveys additional information and for this reason it may be used together with standard and well-accepted methods $[109,110]$ in which the forecasting of aftershocks in time and magnitude domain is carried out by employing the modified Omori law and the Gutenberg-Richter law.

The models discussed in the previous paragraphs do not impose any direct correlation between successive magnitudes apart from those inherited from the combination of the
Gutenberg-Richter law and Omori law, the fact that the rate of triggered events increases with the magnitude of the triggering event, that is, the ETAS model and STAI. It is a fact, however, that magnitude correlations exist in both regional $[17,36,40,111]$ and global $[38,39]$ seismic catalogues. Thus, one should also investigate whether such correlations are responsible for the predictability observed in Figure 9. Particularly, for California these correlations have been extensively studied by Davidsen and Green [96] and Lippiello et al. [112]. Davidsen and Green [96] showed that they cannot be fully accounted for by STAI as was also found in Lippiello et al. [112] (e.g., see their Figure 2). In both of these studies, based on the investigation of the magnitude correlations firstly introduced by Lippiello et al. [113], the common method of investigating such correlations was the construction of the difference $\delta P\left(m_{0}\right)=P\left(\Delta m<m_{0}\right)-P\left(\Delta m^{*}<m_{0}\right)$ between 


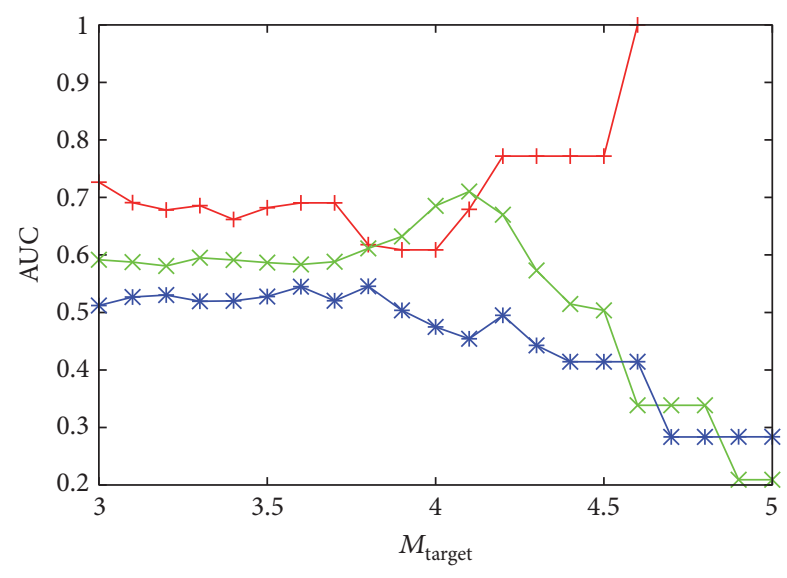

+ Fukuoka $M_{\mathrm{JMA}} 7.2$
$\leftarrow$ Miyagi $M_{\mathrm{JMA}} 6.2$
$+\quad b=1$

(a)

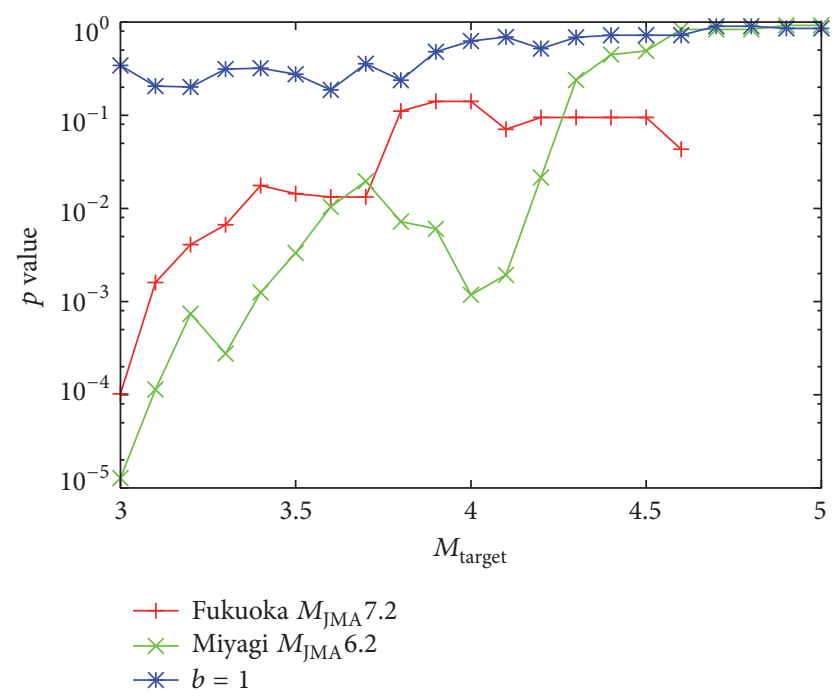

(b)

FIGURE 15: Comparison of (a) AUC and (b) $p$ values obtained when using $e_{k}$ as a predictor for various $M_{\text {target }}$ for the real aftershock magnitude time series of Fukuoka and Miyagi EQs and an ETAS simulation with $b=1$ produced by etasim.f of SASeis2006 [104]. In all cases, $M_{\text {thres }}=2.0$ has been used.

the cumulative distributions of the observed differences between successive EQs $\Delta m \equiv M_{k+1}-M_{k}$ and those obtained $\Delta m^{*} \equiv M_{k+1}^{*}-M_{k}^{*}$ from randomly shuffled copies $\left\{M_{k}^{*}\right\}$ of the original catalogue. Since such correlations are expected [112] to be stronger for EQs which are closer in time and increases with the magnitude threshold of the catalogue used, we focused our attention on the first 24 hours after the mainshock, applied a time-dependent threshold $M_{\text {thres }}\left(M_{m}, t\right)$, and investigated the presence of such correlations. The cumulative distributions $P\left(\Delta m<m_{0}\right)$ and $P\left(\Delta m^{*}<m_{0}\right)$ are depicted by the red (solid) and the green (dashed) curves, respectively, in Figure 16 and they actually differ as it is also shown by the $\delta P\left(m_{0}\right)$ (red solid) curve in Figure 17. Hence, it is of crucial importance to confirm that the predictability of large aftershocks presented in Figure 14 (for the same time period and the same $\left.M_{\text {thres }}\left(M_{m}, t\right)\right)$ is not caused by the phenomenon observed in Figures 16 and 17. For this reason, we employed the method of surrogates proposed by Schreiber and Schmitz [114] as implemented in the TISEAN package [115] and applied it to the magnitude time series. Such a method creates surrogate data with the same Fourier amplitudes and the same distribution of values. This procedure reproduces the behavior found in Figures 16 and 17 (see the blue dashed curve in Figure 16 and the green, blue, and thick black curves in Figure 17). We generated $10^{3}$ such surrogate magnitude time series, analyzed them by the prediction algorithm, and compared their AUCs with the AUCs of the observed magnitude time series that led to Figure 14. The percentage the AUCs of a surrogate time series was greater than the AUC of the observed magnitude time series in the majority of cases shown in Figure 14 are 9.0\%, 0.2\%, 0.6\%, $43 \%, 0.1 \%, 0.1 \%, 0.7 \%$, and $54 \%$ for the cases of the Landers, $\mathrm{El}$ Mayor-Cucapah, Hector Mine, Northridge, 2nd Superstition Hills, Big Bear, 1st Superstition Hills, and Joshua Tree EQs, respectively. Such percentages (cf. if we repeat the procedure for the whole study period the percentages for Northridge and Joshua Tree EQs become $14 \%$ and 32\%, resp.) show that the behavior found in Figures 16 and 17 which is, as mentioned, related to the magnitude correlations found by Davidsen and Green [96] and Lippiello et al. [112] cannot fully account for the observed predictability. Moreover, these results show that the predictability of the aftershock time series is a nonlinear property [116] of the magnitude time series of aftershocks. The latter fact is compatible with the high nonlinearity exhibited by the coherent noise model.

Moreover, as a more direct analysis to avoid the effect of data incompleteness, we excluded the first one day period after the mainshock from the target period of the forecasting, employed time varying threshold $M_{\text {thres }}\left(M_{m}, t\right)$, and examined the predictability in the [1 day, 1 month] time-interval. The corresponding AUCs and $p$ values are shown in Figure 18. These values (apart from those concerning the second Superstition Hills M6.6 EQ) certainly point to the statistical significance of the present method (86 cases out of the 129 lead to $p$ values smaller than $10 \%$ with 79 of them below $5 \%$ ), although they are higher than the $p$ values corresponding to Figure 9. This effect is not incompatible with the behavior exhibited by the coherent noise model. It has been recently shown [21, 22] that the expected avalanche size of the $k$ th avalanche of the coherent noise model in a statistical ensemble of initially identical systems relaxes to the steady state value by following a power-law decay, described by the Tsallis $q$-exponential [117], almost inversely proportional to $k$. When we started our study in the second day after the mainshock, we excluded almost $10 \%$ of the first month aftershocks. These events, however, express the most important part of the offequilibrium behavior which can be best described by the present algorithm as shown in Figure 14. As the order $k$ of the 

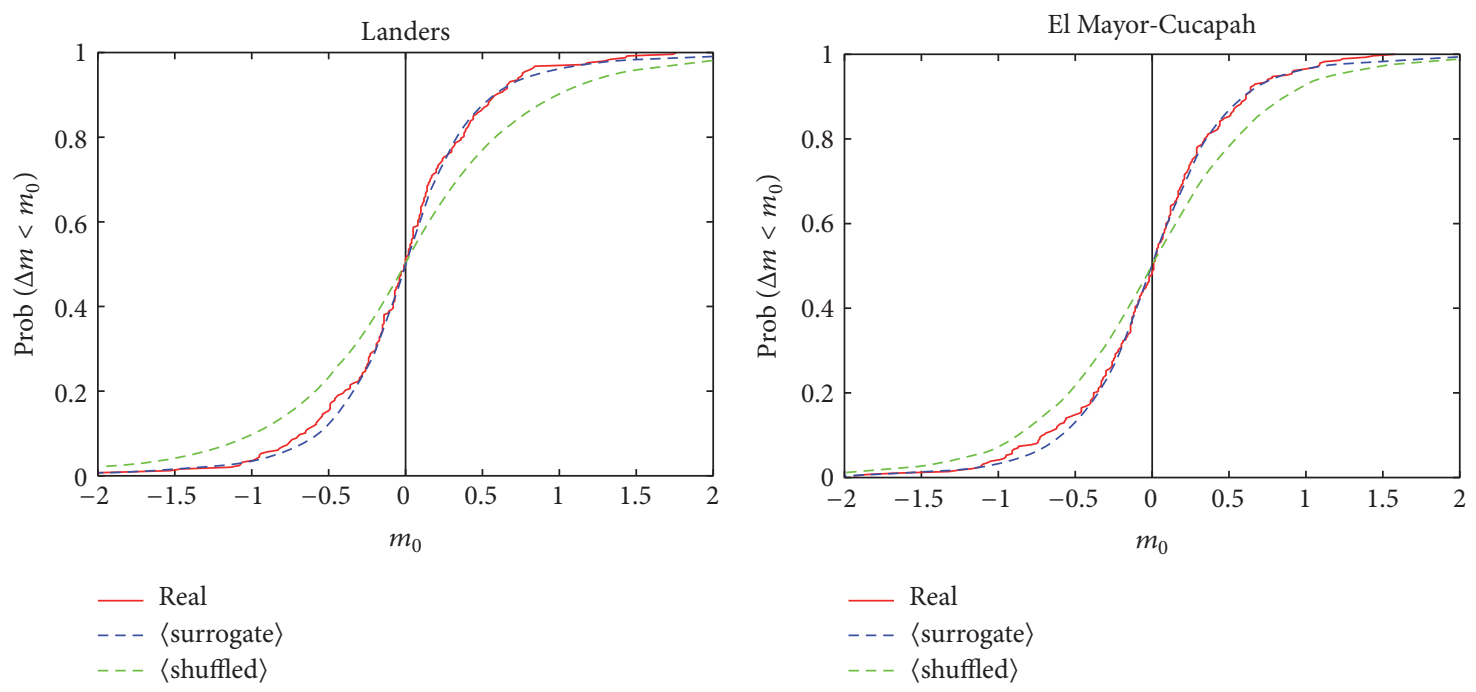

(a)

(b)
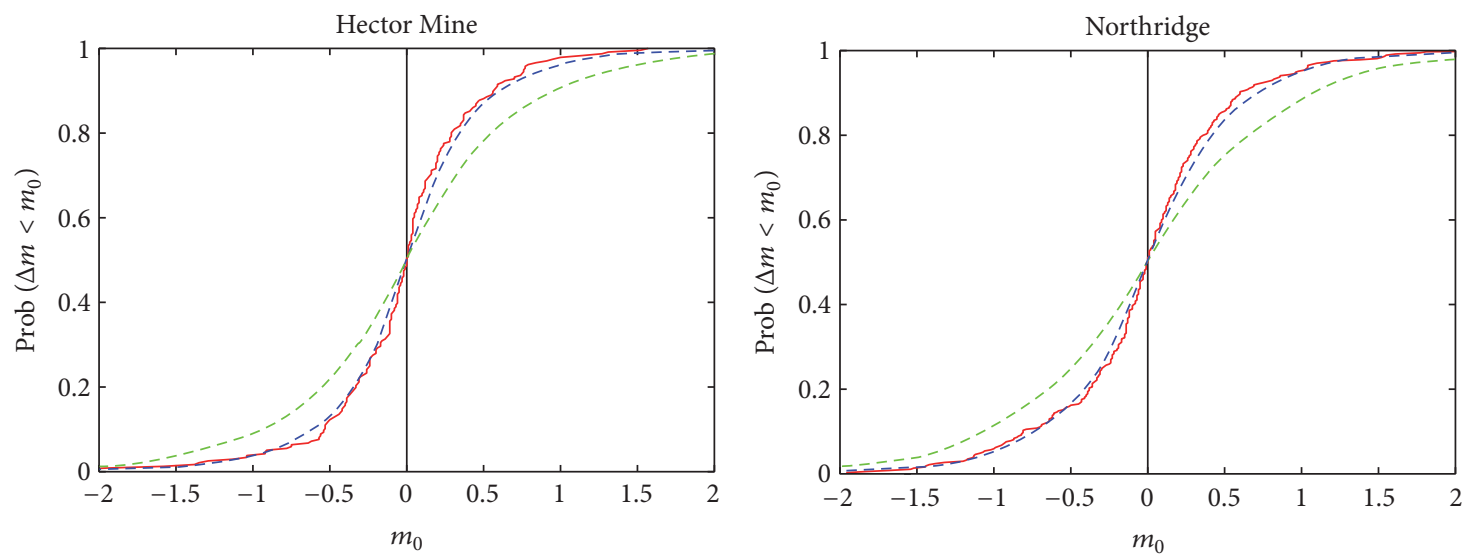

— Real

- Real

- - - 〈surrogate $\rangle$

- - 〈surrogate $\rangle$

- - $\langle$ shuffled $\rangle$

(c)

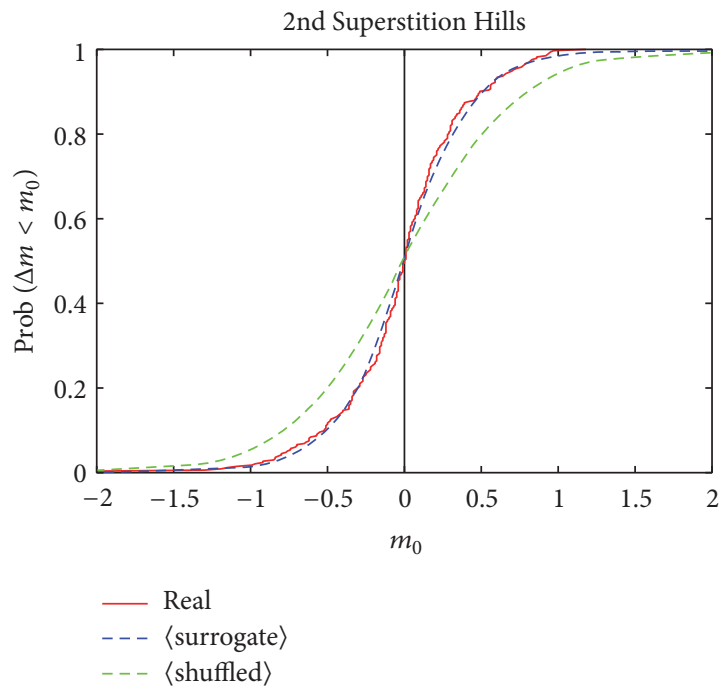

(e)

(d)

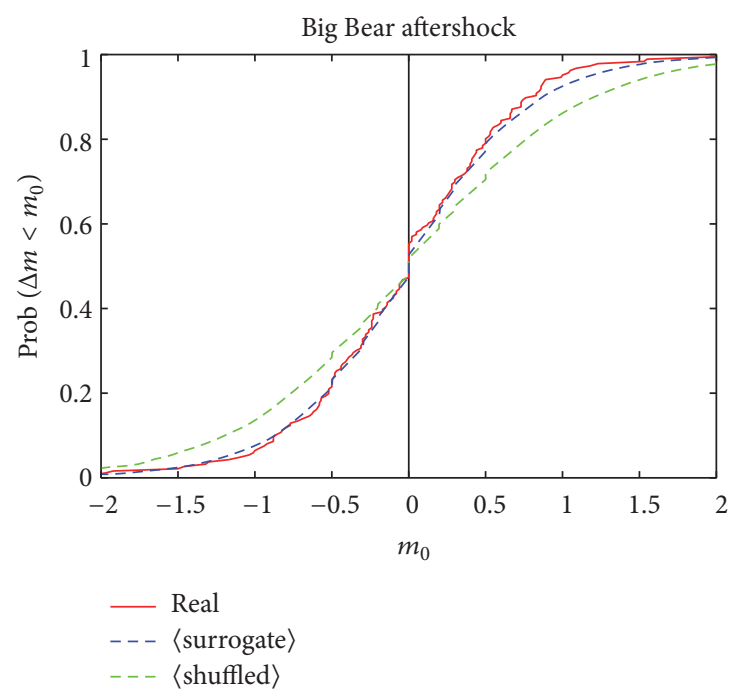

(f)

FIgURE 16: Continued. 


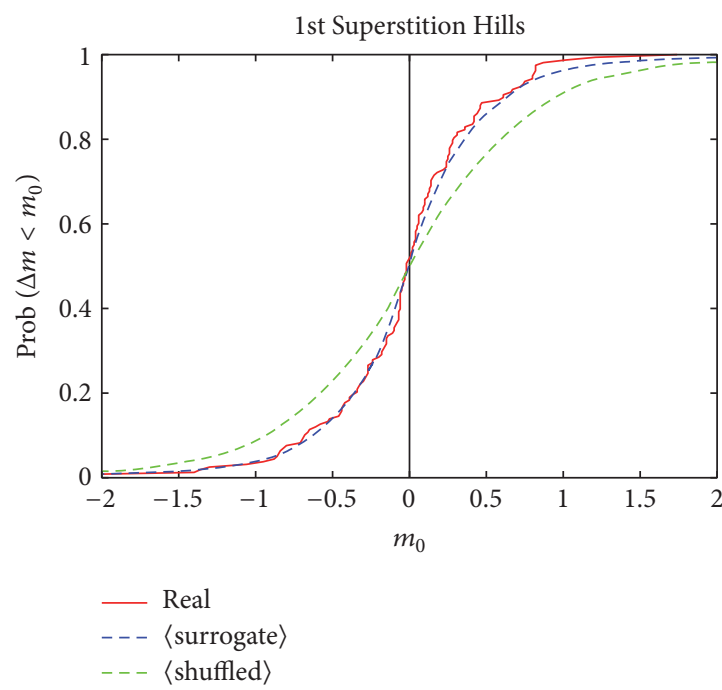

(g)

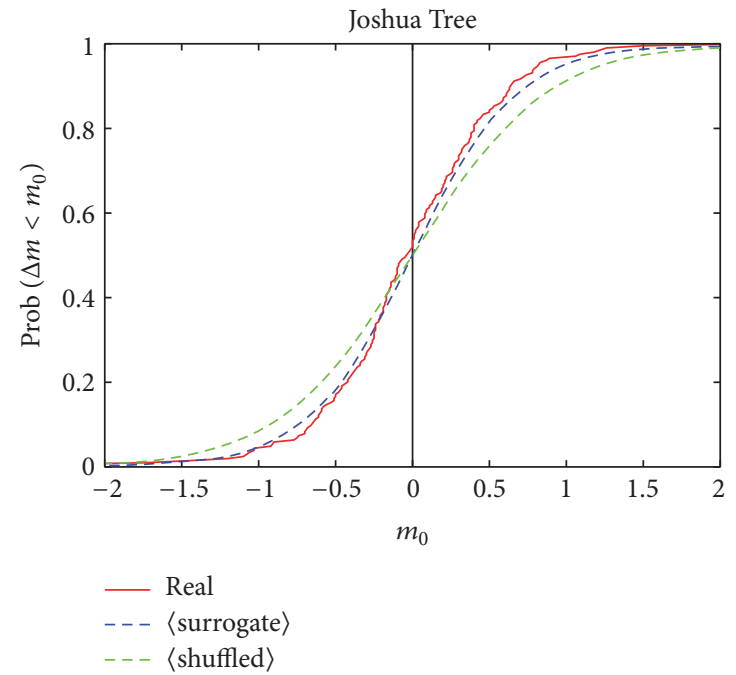

(h)

FIGURE 16: The cumulative distributions of the event increments $\Delta m \equiv M_{k+1}-M_{k}$ for the real aftershocks magnitude time series (solid red) together with those corresponding to randomly shuffled magnitude time series (dashed green) and for surrogate magnitude time series (dashed blue).

avalanche increases, the system deviates less from the steady state and hence it is plausible to assume that its predictability decreases. The fact that the predictability decreases when we exclude the first $10 \%$ of the avalanches produced and focus on the latter $90 \%$ has been also verified by simulations of the coherent noise model.

Thus, in summary, we have shown that the observed predictability of the present algorithm cannot be attributed solely to STAI nor is a result included in up to date ETAS modelling nor is a direct consequence of the magnitude correlations studied by Davidsen and Green [96] and Lippiello et al. [112] in Southern California. The present method tries to predict large aftershocks from the correlation between magnitudes which appear to mimic the behavior of the coherent noise model. According to our view, this is the reason behind the predictability observed in Figure 9 that corresponds to a large time period of study as well as in Figure 14 that corresponds to the first day.

If we focus on the strongest aftershock (which may be the most destructive one), the value of the predictor $e_{k-1}$ before its occurrence for all the 14 EQs studied is $4,3,4,0,2,6$, 1, and 7 for the Landers, El Mayor-Cucapah, Hector Mine, Northridge, second Superstition Hills, Big Bear, first Superstition Hills, and Joshua Tree EQs in California, whereas it is $4,3,0,0,0$, and 0 for the Sumatra-Andaman, Sumatra-Nias, Sumatra Indonesia, Chile, Tohoku Japan, and Indian Ocean EQs in GCMT. Thus, when selecting for Southern California the threshold $e_{t}^{\mathrm{SC}}=7$ and $e_{t}^{\mathrm{GCMT}}=4$ for GCMT, as in Figure 13(b), one could have successfully predicted the strongest aftershock for all the 14 EQs studied with the corresponding operating points above the diagonal in the ROC diagram. Moreover, in 12 out of the 14 cases studied, that is, if we discard the cases of Sumatra-Nias (which, as mentioned, could have been predicted based on the analysis of Sumatra-Andaman) and Indian Ocean EQ, these operating points lie in the left uppermost quadrant of the ROC diagram with an average hit rate $76 \%$ and an average false alarm rate $28 \%$ (cf. the corresponding average values for all $14 \mathrm{EQs}$ are $77 \%$ and $34 \%$, resp.). Similarly, when selecting $e_{t}^{\mathrm{SC}}=5$ and $e_{t}^{\mathrm{GCMT}}=3$ the 12 out the 14 operating points lie in the region $\mathrm{FPr}<0.3$ and $\mathrm{TPr}>0.3$; see Figure 13(a). The 14 operating points in this case lead to an average hit rate $61 \%$ and an average false alarm rate $16 \%$.

Returning now to the results for Southern California, we have to note that the probability to observe $e_{k} \leq 4$ in the examined predictor time series is $0.8 \%, 0.4 \%, 1.2 \%$, and $2.0 \%$ for the four stronger EQs, respectively, thus leading to a number of alarms smaller than $2 \%$ of the observed aftershocks (with $M_{k} \geq 2.0$ ) in each case (cf. the corresponding total alarm duration in conventional time, which is depicted in Figure 19(a), varies from four hours and forty minutes for the Landers EQ to one hour for the El Mayor-Cucapah EQ). Moreover, when considering the five stronger aftershocks in each case (see Table 3 ), we observe that by setting $e_{t}=8$ we can successfully predict (more than) $80 \%$ of these strong aftershocks (cf. only the cases labeled L4, E4, H3b, and N4 exhibit $e_{k-1}>8$ ). As an example, we also plot in Figures 19(b) and 19 (c) the total alarm duration in conventional time as a function of the time elapsed from the mainshock for the predictor thresholds $e_{t}=6$ and 8 . We observe that, during the first month from the mainshock, which is the most highly prone period to strong aftershocks, this quantity varies from 9-30 hours $\left(e_{t}=6\right)$ to 3-4 days $\left(e_{t}=8\right)$.

\section{Conclusions}

In conclusion, in the present study we presented a simple algorithm for predicting aftershock magnitudes based on 

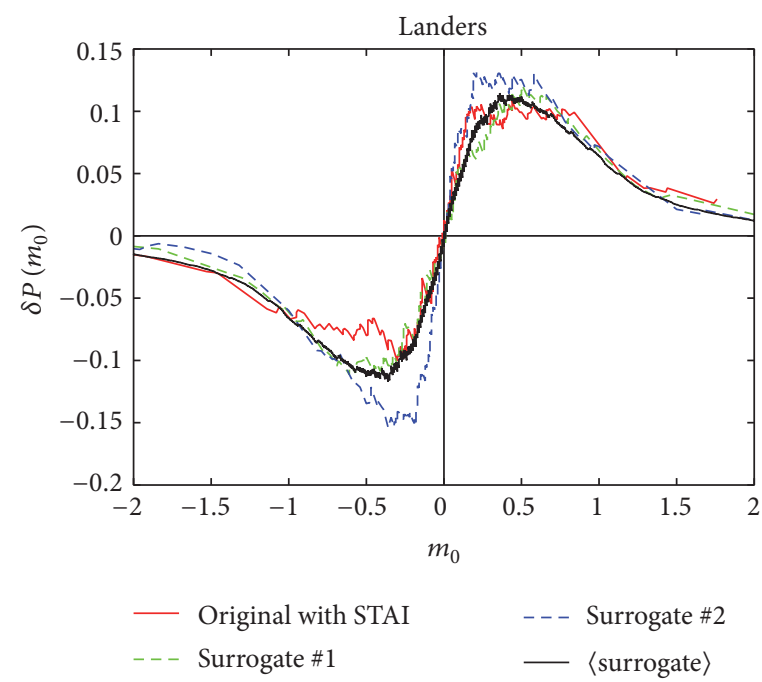

(a)

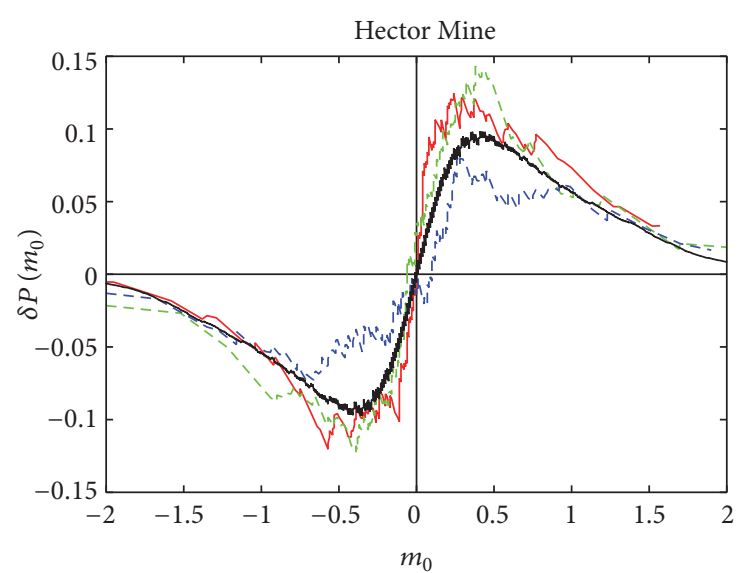

Original with STAI

Surrogate \#1

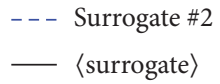

(c)

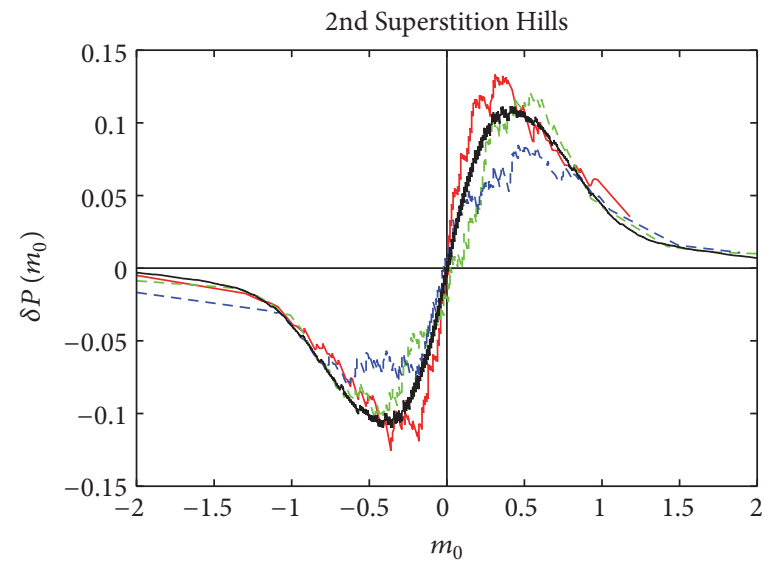

Original with STAI

- - - Surrogate \#2

- - - Surrogate \#1

- 〈surrogate $\rangle$

(e)

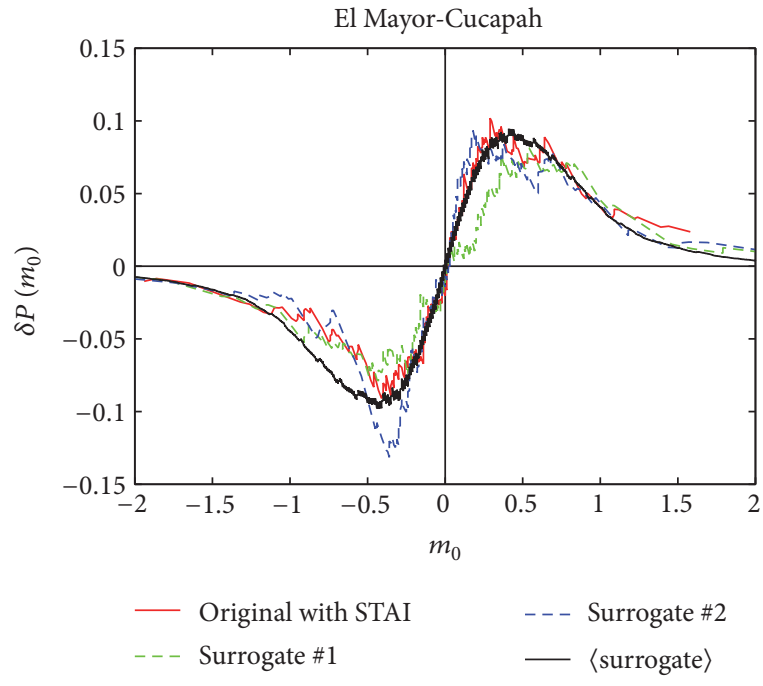

(b)

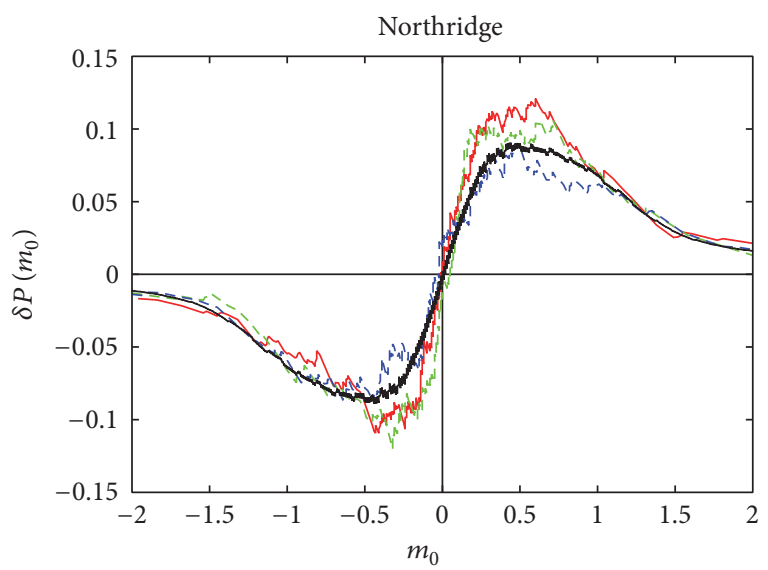

- Original with STAI $\quad$ - - Surrogate $\# 2$

(d)

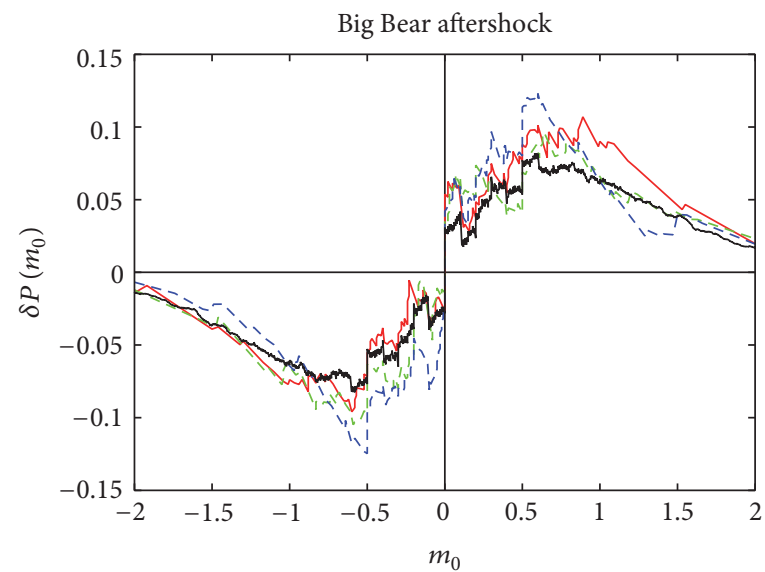

Original with STAI

- - - Surrogate \#2

- - - Surrogate \#1

- 〈surrogate〉

(f)

FIgure 17: Continued. 


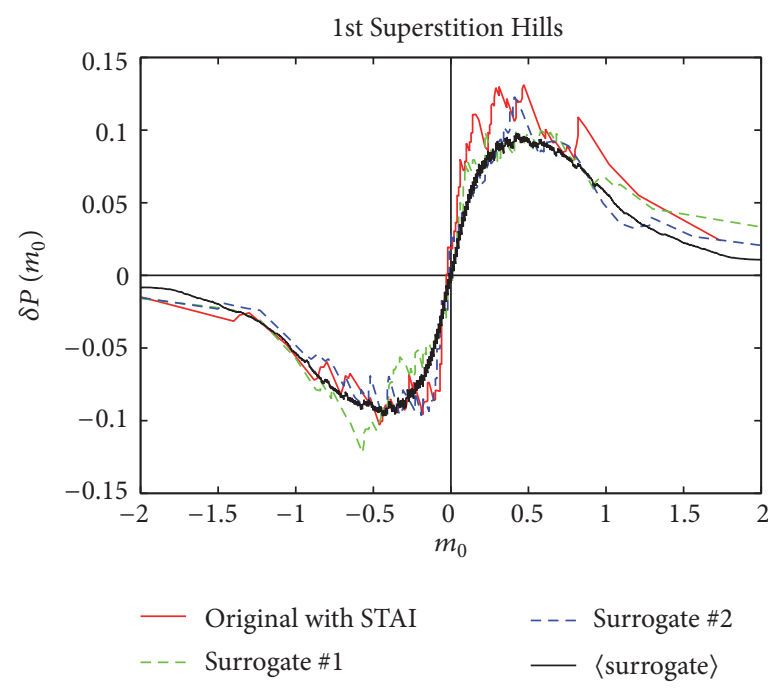

(g)

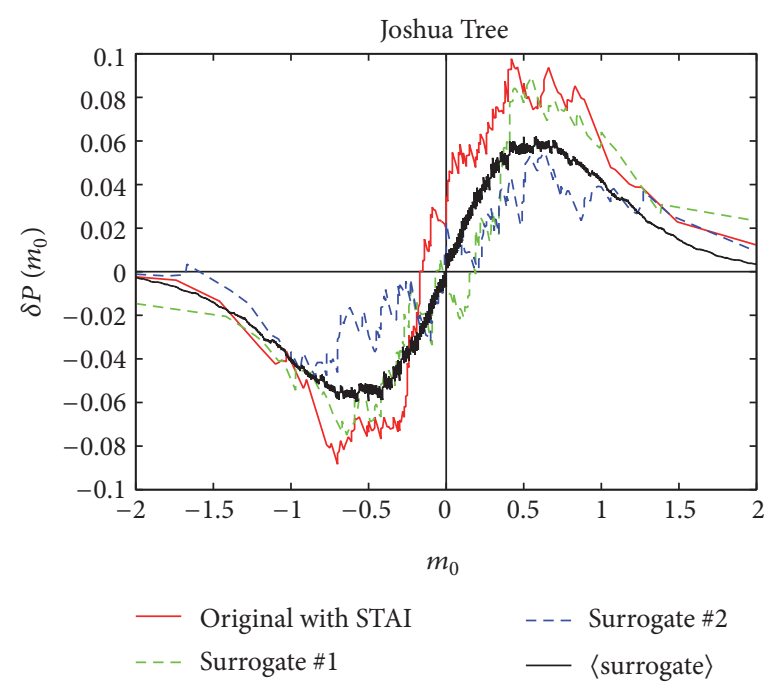

(h)

FIGURE 17: The quantity $\delta P\left(m_{0}\right)$ for the observed aftershocks magnitude time series (solid red) together with the corresponding quantity resulting from two example surrogate time series (dashed green and dashed blue) and the average $\delta P\left(m_{0}\right)$ resulting of the whole ensemble of the surrogate time series (thick black curve).

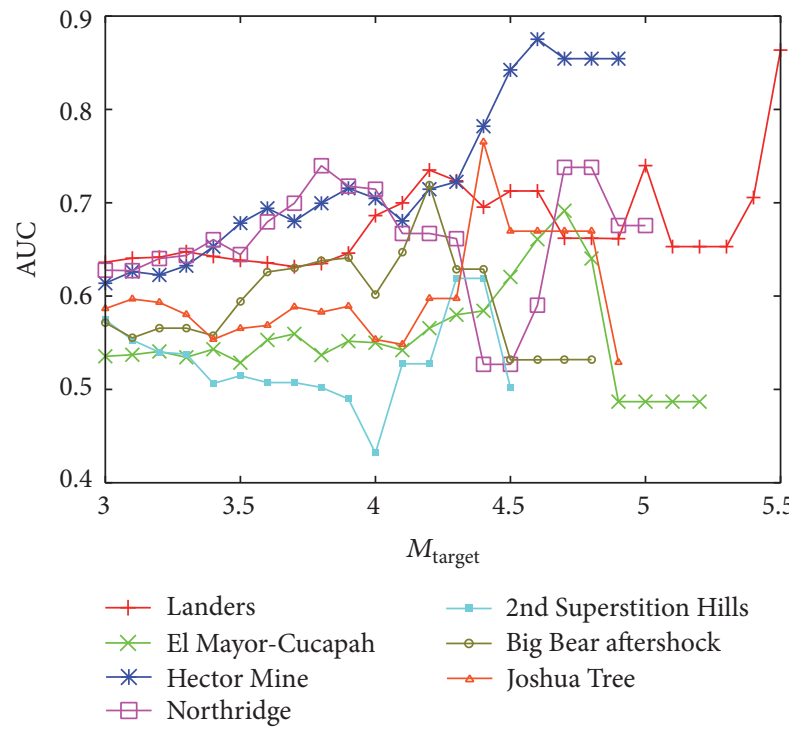

(a)

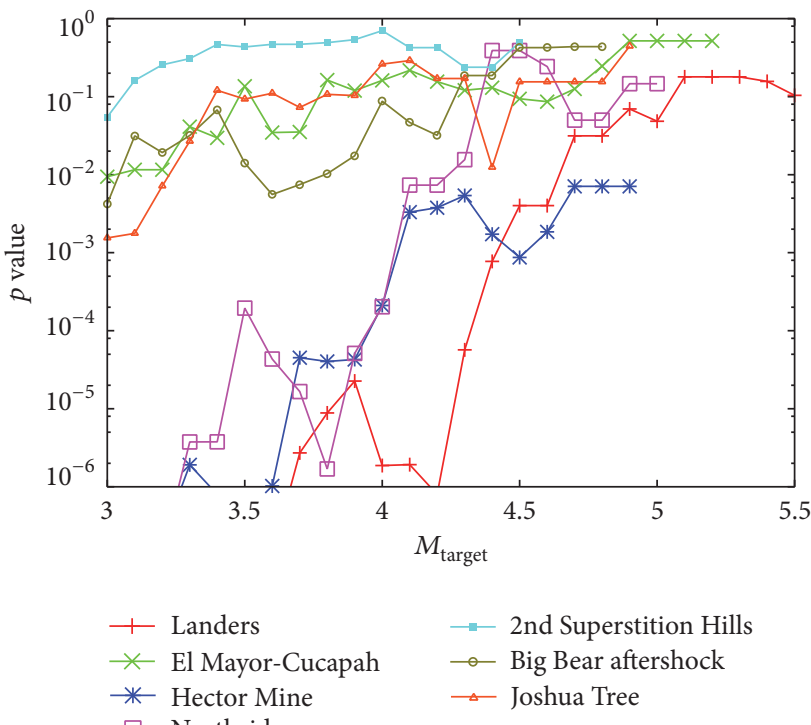

(b)

FIGURE 18: Results obtained when considering as target period the [1 day, 1 month] time-interval after the mainshock and time varying threshold $M_{\text {thres }}\left(M_{m}, t\right)$ from the analysis by ROC diagrams. Panel (a) depicts AUC versus $M_{\text {target }}$ while (b) the corresponding $p$ values.

the analysis of the coherent noise model and examined its performance in Southern California, where a waveform relocated catalogue [8] from 1981 to June 2011 [9] is available. This study has been extended, for comparison purposes, to the six strongest EQs in the Earth during almost the last forty years according to the GCMT catalogue $[100,101]$. The main conclusions are the following:

(1) The predictor time series exhibits the ubiquitous $1 / f$ noise.
(2) The algorithm leads to statistically significant results for 13 out of the $14 \mathrm{EQs}$ studied. Only the case of Sumatra-Nias EQ could be considered by chance, but this EQ might have been anticipated (three and a half days before its occurrence) by the similar analysis for Sumatra-Andaman EQ.

(3) When focusing on predicting aftershocks with $M \geq$ $M_{m}-2$ for all the 14 cases studied, an average behavior with a hit rate $61 \%$ and a false alarm rate of $16 \%$ can 


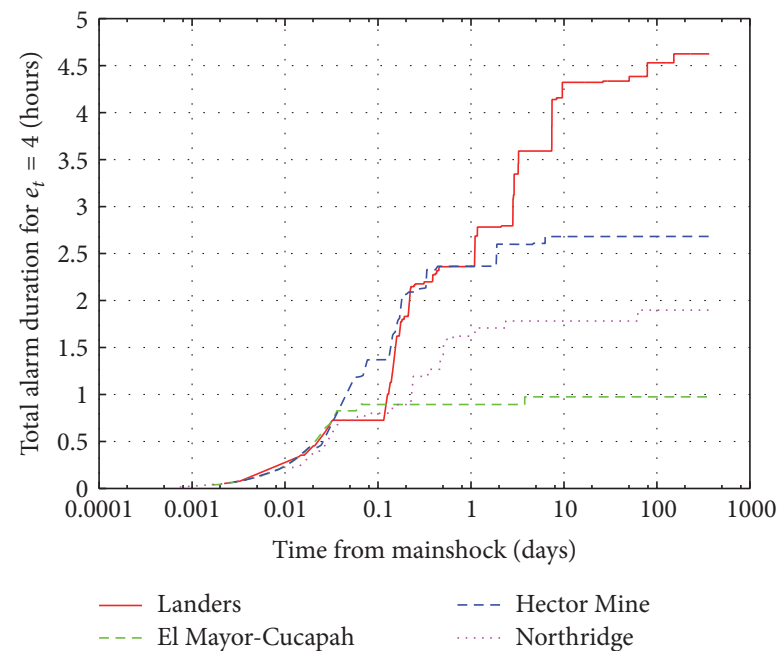

(a)

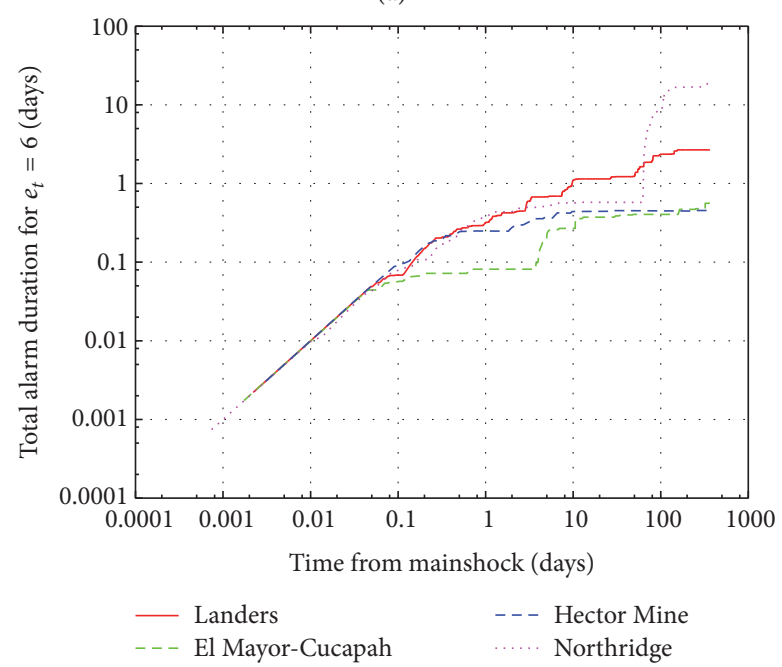

(b)

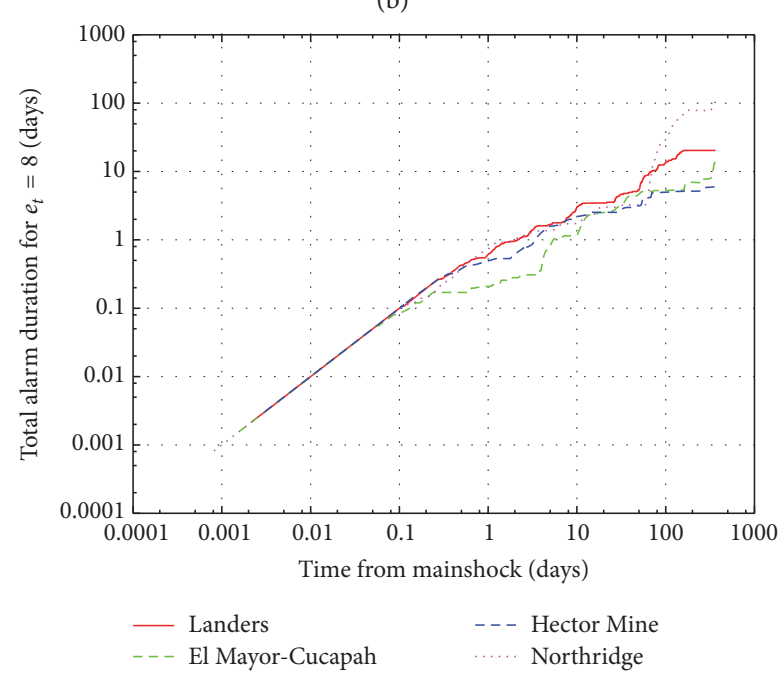

(c)

FIGURE 19: Total alarm duration in conventional time as a function of the time elapsed from the occurrence of the mainshock for the Landers (solid red), El Mayor-Cucapah (dashed green), Hector Mine (dashed blue), and Northridge (dotted magenta) EQs when considering as predictor threshold the value $e_{t}=4(\mathrm{a}), e_{t}=6(\mathrm{~b})$, and $e_{t}=8$ (c).
TABLE 3: The 5 stronger aftershocks as determined by truncating magnitude to the first decimal digit for the four stronger EQs $(M \geq$ 6.7) in Southern California studied (see also Table 1) together with the corresponding values of the predictor $e_{k-1}$.

\begin{tabular}{|c|c|c|c|}
\hline EQ name & $M$ & $e_{k-1}$ & Origin time (UTC) \\
\hline Landers & 7.30 & & $1992 / 06 / 28$ 11:57:33 \\
\hline $\mathrm{L} 1$ & 6.30 & 4 & $1992 / 06 / 28$ 15:05:30 \\
\hline L2 & 5.77 & 0 & 1992/06/28 12:00:44 \\
\hline L3 & 5.70 & 1 & $1992 / 06 / 28$ 12:01:15 \\
\hline $\mathrm{L} 4$ & 5.69 & 9 & 1992/06/29 14:08:37 \\
\hline L5 & 5.53 & 8 & $1992 / 06 / 28$ 14:43:21 \\
\hline El Mayor-Cucapah & 7.20 & & 2010/04/04 22:40:42 \\
\hline E1 & 5.70 & 3 & 2010/04/04 22:50:17 \\
\hline E2 & 5.43 & 3 & 2010/04/04 23:15:13 \\
\hline E3 & 5.38 & 6 & 2010/04/04 23:25:06 \\
\hline $\mathrm{E} 4$ & 5.29 & 11 & $2010 / 04 / 08$ 16:44:25 \\
\hline E5 & 5.26 & 0 & 2010/04/04 22:43:01 \\
\hline Hector Mine & 7.10 & & 1999/10/16 09:46:43 \\
\hline $\mathrm{H} 1$ & 5.77 & 4 & 1999/10/16 09:59:34 \\
\hline $\mathrm{H} 2$ & 5.37 & 4 & $1999 / 10 / 16$ 12:57:20 \\
\hline $\mathrm{H} 3 \mathrm{a}$ & 4.99 & 8 & 1999/10/21 01:54:06 \\
\hline $\mathrm{H} 3 \mathrm{~b}$ & 4.99 & 11 & 1999/10/22 16:08:47 \\
\hline H5a & 4.98 & 4 & 1999/10/21 01:54:33 \\
\hline $\mathrm{H} 5 \mathrm{~b}$ & 4.98 & 3 & 1999/10/21 01:54:28 \\
\hline $\mathrm{H} 5 \mathrm{c}$ & 4.98 & 2 & 1999/10/16 09:52:53 \\
\hline Northridge & 6.70 & & 1994/01/17 12:30:54 \\
\hline $\mathrm{N} 1$ & 5.89 & 0 & 1994/01/17 12:31:56 \\
\hline $\mathrm{N} 2$ & 5.58 & 8 & 1994/01/17 23:33:30 \\
\hline N3 & 5.24 & 4 & 1994/01/18 00:43:08 \\
\hline N4 & 5.24 & 15 & 1994/03/20 21:20:12 \\
\hline N5 & 5.20 & 3 & 1994/01/17 12:40:34 \\
\hline
\end{tabular}

be achieved since the corresponding percentages may fluctuate for each particular case; see Figure 13(a).

(4) The strongest aftershock of each EQ in Southern California with $M_{m} \geq 6.7$ could have been successfully predicted with a number of alarms amounting only $2 \%$ of the total number of aftershocks (with $M \geq 2.0$ ) and a total alarm duration of less than five hours in each case.

The 3 cases when a stronger EQ occurs within or close to the rectangular area studied during the study period of another EQ have revealed the following. The second Superstition Hills EQ would have been missed, whereas Landers and SumatraNias EQs might have been anticipated. The corresponding selection of operating points on the ROC diagram can be seen in Figure 13(b) and leads to an average behavior with a hit rate $77 \%$ and a false alarm rate of $34 \%$. 


\section{Appendix}

\section{A FORTRAN Code Implementing the Algorithm}

The following simple FORTRAN code (Findek.f) calculates the value of $e_{k}$ based on the sequence of EQ magnitudes given in the file input.cat. The user has to provide the number (nmax) of EQs in the catalogue file. The output is then exported to the ASCII file out . dat that contains in each row the aftershock magnitude $M_{k}$ and the value of $e_{k-1}$, that is, the value of $e_{k}$ before its occurrence.

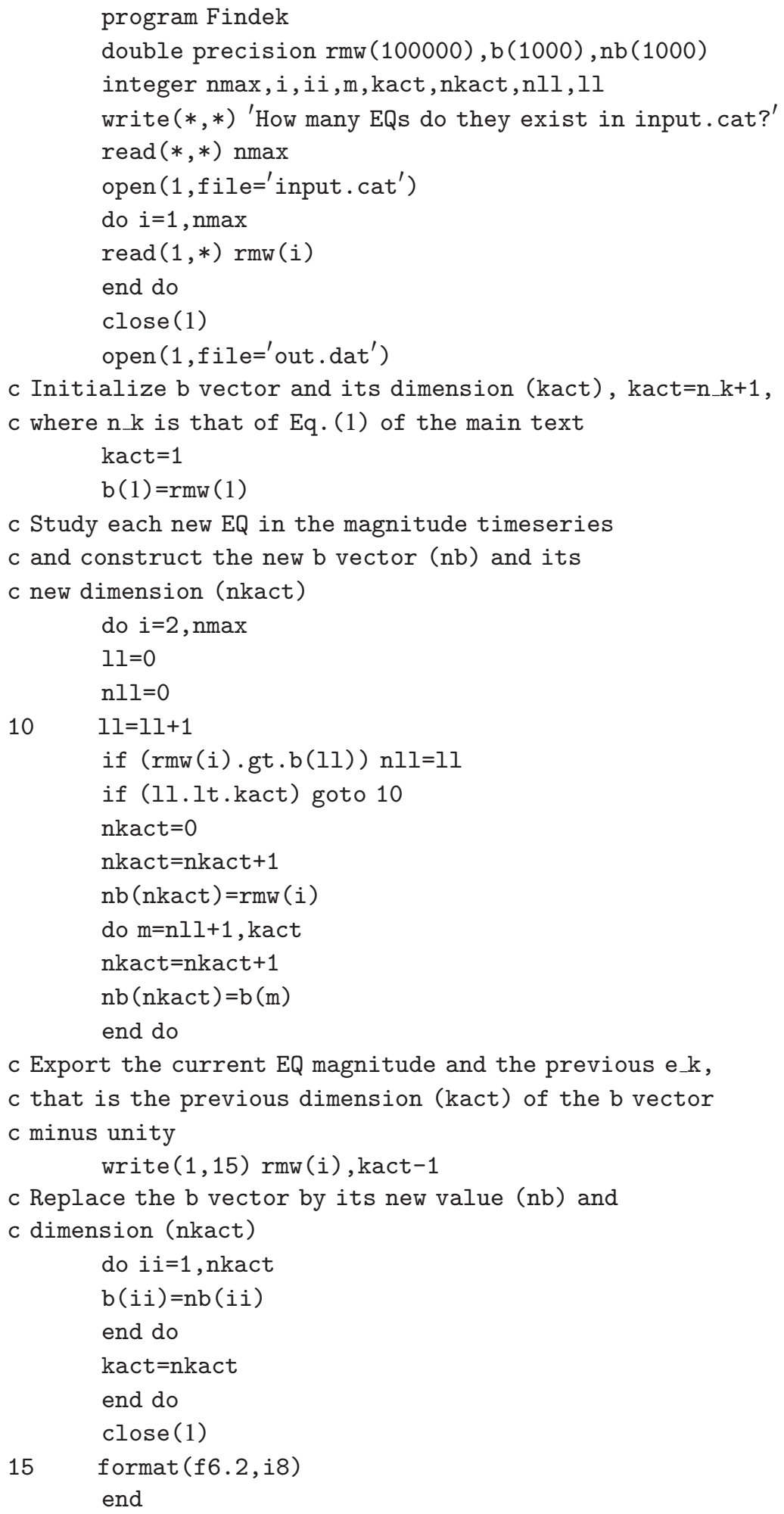


As an example input.cat we provide below the data concerning the Landers EQ that led to the results presented in Figure 5(c) consisting of (nmax=)67 EQs:
7.30
$34.202-116.43519920628115733$
5.77
$34.126-116.40219920628120044$
5.70
$34.123-116.25319920628120115$
5.00
$34.048-116.45619920628120216$
3.50
$34.523-116.61219920628121748$
3.00
$34.175-116.78219920628121850$
4.00
$34.581-116.63319920628122044$
2.20
$34.181-116.44219920628122641$
4.20
$34.477-116.62319920628122736$
5.49
$34.166-116.42019920628123639$
5.41
$34.355-116.52419920628124052$
4.44
$34.120-116.42019920628124358$
4.29
$34.483-116.52719920628125609$
4.00
$34.319-116.49919920628130640$
4.30
$33.910-116.32719920628130647$
3.50
$34.326-116.47419920628130815$
4.87
$34.438-116.45619920628131049$
3.80
$34.321-116.36919920628131336$
4.11
$34.155-116.42319920628131747$
4.50
$34.068-116.43419920628131815$
3.22
$34.128-116.40219920628131924$
3.36
$34.125-116.41719920628132309$
3.68
$34.222-116.43619920628132344$
4.88
$34.175-116.41119920628132604$
3.00
$34.001-116.54319920628133537$
3.00
$34.430-116.49219920628133559$
4.17
$34.190-116.42419920628134054$
3.00
$34.524-116.58219920628134649$
4.22
$34.100-116.37719920628135016$
4.95
$34.143-116.40319920628135044$
4.00
$34.157-116.46919920628135142$
3.50
$34.141-116.43219920628135244$
4.14
$34.118-116.64019920628140928$
3.01
$34.361-116.47119920628141026$
2.50
$34.615-116.62919920628141106$
4.03
$34.609-116.63219920628142901$
2.80
$34.161-116.46819920628142945$
3.00
$34.430-116.45619920628143027$
2.70
$34.155-116.41819920628143045$
3.54
$34.636-116.51419920628143207$
3.40
$34.596-116.65219920628143230$
3.32
$34.279-116.44019920628143533$
4.35
$34.099-116.42919920628143906$
3.00
$34.610-116.63119920628144049$
3.00
$34.164-116.43519920628144309$
5.53
$34.169-116.85319920628144321$
3.37
$34.198-116.86119920628145913$
2.80
$34.089-116.43619920628150000$
4.54
$34.167-116.82119920628150450$
6.30
$34.202-116.82819920628150530$
3.00
$34.199-116.79819920628150803$
2.50
$34.203-116.80119920628150841$
2.50
$34.221-116.84719920628150956$
3.00
$34.198-116.77619920628151025$
2.80
$34.138-116.85619920628151102$
3.00
$34.167-116.83919920628151137$
2.50
$34.252-116.72319920628151216$
2.50
$34.288-116.71619920628151337$
3.00
$34.157-116.87019920628151532$
2.80
$34.144-116.84319920628151659$
4.00
$34.164-116.85119920628151709$
4.66
$34.134-116.85119920628151712$
4.58
$34.214-116.74919920628151832$
4.00
$34.051-116.39919920628152016$
4.76
$34.219-116.76219920628152428$
4.27
$34.223-116.80319920628152519$
3.50
$34.217-116.75119920628152653$

Only the first column is used by Findek. $f$, whereas the other three columns correspond to the latitude, longitude, and time of occurrence UTC (with format: YYYYMMDDhhmmss, where YYYY, MM, DD, hh, mm, and ss stand for the year, month, day, hour, minute, and seconds, resp.) of each EQ-within the area studied; see the first row of Table 1 and Figure 1 -according to the waveform relocated catalogue [8] for Southern California from 1981 to June 2011 [9] and have been included here as test data.

\section{Competing Interests}

The authors declare that there is no conflict of interests regarding the publication of this paper.

\section{Acknowledgments}

The authors would like to thank Professor Egill Hauksson for his suggestions on the use of the waveform relocated catalogue of Southern California (1981 to June 2011). 


\section{References}

[1] N. V. Sarlis and S.-R. G. Christopoulos, "Predictability of the coherent-noise model and its applications," Physical Review E, vol. 85, no. 5, Article ID 051136, 2012.

[2] M. E. J. Newman and K. Sneppen, "Avalanches, scaling, and coherent noise," Physical Review E, vol. 54, no. 6, pp. 6226-6231, 1996.

[3] K. Sneppen and M. E. J. Newman, "Coherent noise, scale invariance and intermittency in large systems," Physica D: Nonlinear Phenomena, vol. 110, no. 3-4, pp. 209-222, 1997.

[4] M. E. J. Newman, "Self-organized criticality, evolution and the fossil extinction record," Proceedings of the Royal Society of London B: Biological Sciences, vol. 263, no. 1376, pp. 1605-1610, 1996.

[5] P. A. Varotsos, N. V. Sarlis, and E. S. Skordas, "Spatio-Temporal complexity aspects on the interrelation between Seismic Electric Signals and Seismicity," Practica of Athens Academy, vol. 76, pp. 294-321, 2001.

[6] P. A. Varotsos, N. V. Sarlis, and E. S. Skordas, "Long-range correlations in the electric signals that precede rupture," Physical Review E, vol. 66, no. 1, Article ID 011902, 2002.

[7] P. A. Varotsos, N. V. Sarlis, and E. S. Skordas, Natural Time Analysis: The New View of Time. Precursory Seismic Electric Signals, Earthquakes and other Complex Time-Series, Springer, Berlin, Germany, 2011.

[8] E. Hauksson, W. Yang, and P. M. Shearer, "Waveform relocated earthquake catalog for southern California (1981 to June 2011)," Bulletin of the Seismological Society of America, vol. 102, no. 5, pp. 2239-2244, 2012.

[9] E. Hauksson, W. Yang, and P. M. Shearer, "Waveform Relocated Earthquake Catalog for Southern California (1981 to 2011)," 2011, http://www.data.scec.org/research-tools/alt-2011-dd-haukssonyang-shearer.html.

[10] C. Wilke, S. Altmeyer, and T. Martinetz, "Aftershocks in coherent-noise models," Physica D: Nonlinear Phenomena, vol. 120, no. 3-4, pp. 401-417, 1998.

[11] U. Tirnakli and S. Abe, "Aging in coherent noise models and natural time," Physical Review E, vol. 70, no. 5, Article ID 056120, 2004.

[12] T. Utsu, "A statistical study of the occurrence of aftershocks," Geophysical Magazine, vol. 30, pp. 521-605, 1961.

[13] R. Shcherbakov, D. L. Turcotte, and J. B. Rundle, "A generalized Omori's law for earthquake aftershock decay," Geophysical Research Letters, vol. 31, no. 11, Article ID L11613, 2004.

[14] J. Davidsen, C. Gu, and M. Baiesi, "Generalized Omori-Utsu law for aftershock sequences in southern California," Geophysical Journal International, vol. 201, no. 2, pp. 965-978, 2015.

[15] A. Celikoglu and U. Tirnakli, "Unified scaling law in the coherent noise model," Physica A: Statistical Mechanics and its Applications, vol. 392, no. 19, pp. 4543-4548, 2013.

[16] P. Bak, K. Christensen, L. Danon, and T. Scanlon, "Unified scaling law for earthquakes," Physical Review Letters, vol. 88, no. 17, Article ID 178501, 2002.

[17] P. A. Varotsos, N. V. Sarlis, E. S. Skordas, H. K. Tanaka, and M. S. Lazaridou, "Entropy of seismic electric signals: analysis in natural time under time reversal," Physical Review E, vol. 73, no. 3, Article ID 021123, 2006.

[18] P. Varotsos, N. V. Sarlis, E. S. Skordas, S. Uyeda, and M. Kamogawa, "Natural time analysis of critical phenomena," Proceedings of the National Academy of Sciences of the United States of America, vol. 108, no. 28, pp. 11361-11364, 2011.
[19] F. Vallianatos, G. Michas, P. Benson, and P. Sammonds, "Natural time analysis of critical phenomena: the case of acoustic emissions in triaxially deformed Etna basalt," Physica A: Statistical Mechanics and its Applications, vol. 392, no. 20, pp. 5172-5178, 2013.

[20] E. Flores-Márquez, C. Vargas, L. Telesca, and A. Ramírez-Rojas, "Analysis of the distribution of the order parameter of synthetic seismicity generated by a simple spring-block system with asperities," Physica A: Statistical Mechanics and Its Applications, vol. 393, pp. 508-512, 2014.

[21] S.-R. G. Christopoulos and N. V. Sarlis, "q-Exponential relaxation of the expected avalanche size in the coherent noise model," Physica A: Statistical Mechanics and Its Applications, vol. 407, pp. 216-225, 2014.

[22] S.-R. Christopoulos and N. Sarlis, "Corrigendum to 'qexponential relaxation of the expected avalanche size in the coherent noise model' [Physica A 407 (2014) 216-225]," Physica A: Statistical Mechanics and Its Applications, vol. 438, p. 667, 2015.

[23] D. Tsuji and H. Katsuragi, "Temporal analysis of acoustic emission from a plunged granular bed," Physical Review E, vol. 92, no. 4, Article ID 042201, 2015.

[24] P. A. Varotsos, N. V. Sarlis, E. S. Skordas, and M. S. Lazaridou, "Identifying sudden cardiac death risk and specifying its occurrence time by analyzing electrocardiograms in natural time," Applied Physics Letters, vol. 91, no. 6, Article ID 064106, 2007.

[25] N. V. Sarlis, E. S. Skordas, and P. A. Varotsos, "Heart rate variability in natural time and $1 / \mathrm{f}$ 'noise"' $E P L$, vol. 87 , no. 1 , Article ID 18003, 2009.

[26] N. Papasimakis and F. Pallikari, "Correlated and uncorrelated heart rate fluctuations during relaxing visualization," $E P L$, vol. 90, no. 4, Article ID 48003, 2010.

[27] N. V. Sarlis, S.-R. G. Christopoulos, and M. M. Bemplidaki, "Change $\Delta S$ of the entropy in natural time under time reversal: complexity measures upon change of scale," EPL, vol. 109, no. 1, Article ID 18002, 2015.

[28] P. A. Varotsos, N. V. Sarlis, and E. S. Skordas, "Detrended fluctuation analysis of the magnetic and electric field variations that precede rupture," Chaos, vol. 19, no. 2, Article ID 023114, 2009.

[29] A. Ramírez-Rojas, L. Telesca, and F. Angulo-Brown, "Entropy of geoelectrical time series in the natural time domain," Natural Hazards and Earth System Science, vol. 11, no. 1, pp. 219-225, 2011.

[30] S. M. Potirakis, A. Karadimitrakis, and K. Eftaxias, "Natural time analysis of critical phenomena: the case of pre-fracture electromagnetic emissions," Chaos, vol. 23, no. 2, Article ID 023117, 2013.

[31] E. S. Skordas and N. V. Sarlis, "On the anomalous changes of seismicity and geomagnetic field prior to the $2011 \mathrm{Mw} 9.0$ Tohoku earthquake," Journal of Asian Earth Sciences, vol. 80, pp. 161-164, 2014.

[32] C. Vargas, E. Flores-Márquez, A. Ramírez-Rojas, and L. Telesca, "Analysis of natural time domain entropy fluctuations of synthetic seismicity generated by a simple stick-slip system with asperities," Physica A: Statistical Mechanics and Its Applications, vol. 419, pp. 23-28, 2015.

[33] C. A. Varotsos and C. Tzanis, "A new tool for the study of the ozone hole dynamics over Antarctica," Atmospheric Environment, vol. 47, pp. 428-434, 2012.

[34] C. A. Varotsos and C. Tzanis, "A new El Niño-Southern Oscillation forecasting tool based on Southern Oscillation Index," 
Atmospheric Chemistry and Physics Discussions, vol. 12, no. 7, pp. 17443-17463, 2012.

[35] H. Tanaka, P. A. Varotsos, N. V. Sarlis, and E. S. Skordas, "A plausible universal behaviour of earthquakes in the natural time-domain," Proceedings of the Japan Academy Series B: Physical and Biological Sciences, vol. 80, no. 6, pp. 283-289, 2004.

[36] N. V. Sarlis, E. S. Skordas, and P. A. Varotsos, "Multiplicative cascades and seismicity in natural time," Physical Review E, vol. 80, no. 2, Article ID 022102, 2009.

[37] S. Lennartz, A. Bunde, and D. L. Turcotte, "Modelling seismic catalogues by cascade models: do we need long-term magnitude correlations?" Geophysical Journal International, vol. 184, no. 3, pp. 1214-1222, 2011.

[38] N. V. Sarlis, "Magnitude correlations in global seismicity," Physical Review E, vol. 84, no. 2, Article ID 022101, 2011.

[39] N. V. Sarlis and S.-R. G. Christopoulos, "Natural time analysis of the Centennial Earthquake Catalog," Chaos, vol. 22, no. 2, Article ID 023123, 2012.

[40] N. V. Sarlis, E. S. Skordas, and P. A. Varotsos, "Nonextensivity and natural time: the case of seismicity," Physical Review E, vol. 82, no. 2, Article ID 021110, 2010.

[41] N. V. Sarlis, E. S. Skordas, and P. A. Varotsos, "Order parameter fluctuations of seismicity in natural time before and after mainshocks," EPL, vol. 91, no. 5, Article ID 59001, 2010.

[42] P. Varotsos, N. Sarlis, and E. Skordas, "Scale-specific order parameter fluctuations of seismicity in natural time before mainshocks," EPL, vol. 96, no. 5, Article ID 59002, 2011.

[43] P. A. Varotsos, N. V. Sarlis, E. S. Skordas, and M. S. Lazaridou, "Seismic electric signals: an additional fact showing their physical interconnection with seismicity," Tectonophysics, vol. 589, pp. 116-125, 2013.

[44] N. V. Sarlis, E. S. Skordas, P. A. Varotsos et al., "Minimum of the order parameter fluctuations of seismicity before major earthquakes in Japan," Proceedings of the National Academy of Sciences of the United States of America, vol. 110, no. 34, pp. 13734-13738, 2013.

[45] A. Ramírez-Rojas and E. Flores-Márquez, "Order parameter analysis of seismicity of the Mexican Pacific coast," Physica A: Statistical Mechanics and Its Applications, vol. 392, no. 10, pp. 2507-2512, 2013.

[46] F. Vallianatos, G. Michas, and G. Papadakis, "Non-extensive and natural time analysis of seismicity before the Mw6.4, October 12, 2013 earthquake in the South West segment of the Hellenic Arc," Physica A: Statistical Mechanics and its Applications, vol. 414, pp. 163-173, 2014.

[47] F. Vallianatos, G. Michas, and G. Hloupis, "Multiresolution wavelets and natural time analysis before the January-February 2014 Cephalonia (Mw6.1 \& 6.0) sequence of strong earthquake events," Physics and Chemistry of the Earth, vol. 85-86, pp. 201209, 2016.

[48] S. Uyeda, M. Kamogawa, and H. Tanaka, "Analysis of electrical activity and seismicity in the natural time domain for the volcanic-seismic swarm activity in 2000 in the Izu Island region, Japan," Journal of Geophysical Research, vol. 114, Article ID B02310, 2009.

[49] N. V. Sarlis, E. S. Skordas, P. A. Varotsos, T. Nagao, M. Kamogawa, and S. Uyeda, "Spatiotemporal variations of seismicity before major earthquakes in the Japanese area and their relation with the epicentral locations," Proceedings of the National Academy of Sciences of the United States of America, vol. 112, no. 4, pp. 986-989, 2015.
[50] Q. Huang, "Forecasting the epicenter of a future major earthquake," Proceedings of the National Academy of Sciences of the United States of America, vol. 112, no. 4, pp. 944-945, 2015.

[51] N. V. Sarlis, S.-R. G. Christopoulos, and E. S. Skordas, "Minima of the fluctuations of the order parameter of global seismicity," Chaos, vol. 25, no. 6, Article ID 063110, 2015.

[52] S. M. Potirakis, Y. Contoyiannis, N. S. Melis et al., "Recent seismic activity at Cephalonia (Greece): a study through candidate electromagnetic precursors in terms of non-linear dynamics," Nonlinear Processes in Geophysics, vol. 23, no. 4, pp. 223-240, 2016.

[53] K. W. Hudnut, L. Seeber, and J. Pacheco, "Cross-fault triggering in the November 1987 Superstition Hills earthquake sequence, southern California," Geophysical Research Letters, vol. 16, no. 2, pp. 199-202, 1989.

[54] E. Hauksson, L. M. Jones, K. Hutton, and D. Eberhart-Phillips, "The 1992 Landers Earthquake Sequence: seismological observations," Journal of Geophysical Research: Solid Earth, vol. 98, no. B11, pp. 19835-19858, 1993.

[55] R. A. Bennett, R. E. Reilinger, W. Rodi, Y. Li, M. N. Toksöz, and K. Hudnut, "Coseismic fault slip associated with the 1992 Mw 6.1 Joshua Tree, California, earthquake: Implications for the Joshua Tree-Landers earthquake sequence," Journal of Geophysical Research: Solid Earth, vol. 100, no. B4, pp. 64436461, 1995.

[56] L. Knopoff, T. Levshina, V. I. Keilis-Borok, and C. Mattoni, "Increased long-range intermediate-magnitude earthquake activity prior to strong earthquakes in California," Journal of Geophysical Research: Solid Earth, vol. 101, no. B3, pp. 5779-5796, 1996.

[57] T. Utsu and A. Seki, "A relation between the area of aftershock region and the energy of main shock," Journal of the Seismological Society of Japan, vol. 7, pp. 233-240, 1954.

[58] S. Abe and N. Suzuki, "Aging and scaling of earthquake aftershocks," Physica A: Statistical Mechanics and its Applications, vol. 332, no. 1-4, pp. 533-538, 2004.

[59] T. Geisel, A. Zacherl, and G. Radons, "Generic $1 / f$ noise in chaotic Hamiltonian dynamics," Physical Review Letters, vol. 59, no. 22, pp. 2503-2506, 1987.

[60] T. Geisel, A. Zacherl, and G. Radons, "Chaotic diffusion and $1 / f$-noise of particles in two-dimensional solids," Zeitschrift für Physik B Condensed Matter, vol. 71, no. 1, pp. 117-127, 1988.

[61] D. L. Gilden, T. Thornton, and M. W. Mallon, "1/f noise in human cognition," Science, vol. 67, no. 5205, pp. 1837-1839, 1995.

[62] H. Hennig, R. Fleischmann, and T. Geisel, "Musical rhythms: the science of being slightly off," Physics Today, vol. 65, no. 7, pp. 64-65, 2012.

[63] D. J. Levitin, P. Chordia, and V. Menon, "Musical rhythm spectra from Bach to Joplin obey a $1 / f$ power law," Proceedings of the National Academy of Sciences of the United States of America, vol. 109, no. 10, pp. 3716-3720, 2012.

[64] G. Molchan, "Strategies in strong earthquake prediction," Physics of the Earth and Planetary Interiors, vol. 61, no. 1-2, pp. 84-98, 1990.

[65] G. M. Molchan, "Structure of optimal strategies in earthquake prediction," Tectonophysics, vol. 193, no. 4, pp. 267-276, 1991.

[66] G. M. Molchan, "Earthquake prediction as a decision-making problem," Pure and Applied Geophysics, vol. 149, no. 1, pp. 233247, 1997.

[67] C.-K. Peng, S. V. Buldyrev, S. Havlin, M. Simons, H. E. Stanley, and A. L. Goldberger, "Mosaic organization of DNA 
nucleotides," Physical Review E, vol. 49, no. 2, pp. 1685-1689, 1994.

[68] C.-K. Peng, S. Havlin, H. E. Stanley, and A. L. Goldberger, "Quantification of scaling exponents and crossover phenomena in nonstationary heartbeat time series," Chaos, vol. 5, no. 1, pp. 82-87, 1995.

[69] A. L. Goldberger, L. A. Amaral, L. Glass et al., "PhysioBank, PhysioToolkit, and PhysioNet: components of a new research resource for complex physiologic signals," Circulation, vol. 101, no. 23, pp. E215-E220, 2000.

[70] C.-K. Peng, S. V. Buldyrev, A. L. Goldberger, S. Havlin, M. Simons, and H. E. Stanley, "Finite-size effects on long-range correlations: implications for analyzing DNA sequences," Physical Review E, vol. 47, no. 5, pp. 3730-3733, 1993.

[71] S. Havlin, S. V. Buldyrev, A. L. Goldberger et al., "Statistical and linguistic features of DNA sequences," Fractals, vol. 3, no. 2, pp. 269-284, 1995.

[72] H. E. Stanley, S. V. Buldyrev, A. L. Goldberger, S. Havlin, C.K. Peng, and M. Simons, "Scaling features of noncoding DNA," Physica A: Statistical Mechanics and Its Applications, vol. 273, no. 1-2, pp. 1-18, 1999.

[73] Y. Ashkenazy, P. C. Ivanov, S. Havlin et al., "Decomposition of heartbeat time series: scaling analysis of the sign sequence," Computers in Cardiology, vol. 27, pp. 139-142, 2000.

[74] K. Ivanova and M. Ausloos, "Application of the detrended fluctuation analysis (DFA) method for describing cloud breaking," Physica A: Statistical Mechanics and its Applications, vol. 274, no. 1, pp. 349-354, 1999.

[75] M. Lovallo, A. Shaban, T. Darwich, and L. Telesca, "Investigating the time dynamics of monthly rainfall time series observed in northern Lebanon by means of the detrended fluctuation analysis and the Fisher-Shannon method," Acta Geophysica, vol. 61, no. 6, pp. 1538-1555, 2013.

[76] C. A. Varotsos, I. Melnikova, M. N. Efstathiou, and C. Tzanis, "1/f Noise in the UV solar spectral irradiance," Theoretical and Applied Climatology, vol. 111, no. 3-4, pp. 641-648, 2013.

[77] C. A. Varotsos, S. Lovejoy, N. V. Sarlis, C. G. Tzanis, and M. N. Efstathiou, "On the scaling of the solar incident flux," Atmospheric Chemistry and Physics, vol. 15, no. 13, pp. 73017306, 2015.

[78] L. Telesca and M. Lovallo, "Non-uniform scaling features in central Italy seismicity: a non-linear approach in investigating seismic patterns and detection of possible earthquake precursors," Geophysical Research Letters, vol. 36, no. 1, Article ID L01308, 2009.

[79] L. Telesca, M. Lovallo, A. E.-E. Amin Mohamed et al., "Investigating the time-scaling behavior of the 2004-2010 seismicity of Aswan area (Egypt) by means of the Allan factor statistics and the detrended fluctuation analysis," Natural Hazards and Earth System Science, vol. 12, no. 5, pp. 1267-1276, 2012.

[80] Y. H. Liu, P. Cizeau, M. Meyer, C.-K. Peng, and H. E. Stanley, "Correlations in economic time series," Physica A: Statistical Mechanics and Its Applications, vol. 245, no. 3-4, pp. 437-440, 1997.

[81] Y. Liu, P. Gopikrishnan, P. Cizeau, M. Meyer, C.-K. Peng, and H. E. Stanley, "Statistical properties of the volatility of price fluctuations," Physical Review E, vol. 60, no. 2, pp. 1390-1400, 1999.

[82] N. Vandewalle and M. Ausloos, "Coherent and random sequences in financial fluctuations," Physica A: Statistical Mechanics and its Applications, vol. 246, no. 3-4, pp. 454-459, 1997.
[83] N. Vandewalle and M. Ausloos, "Crossing of two mobile averages: a method for measuring the roughness exponent," Physical Review E, vol. 58, pp. 6832-6834, 1998.

[84] M. Ausloos, N. Vandewalle, P. Boveroux, A. Minguet, and K. Ivanova, "Applications of statistical physics to economic and financial topics," Physica A: Statistical Mechanics and its Applications, vol. 274, no. 1, pp. 229-240, 1999.

[85] P. C. Ivanov, A. Yuen, B. Podobnik, and Y. Lee, "Common scaling patterns in intertrade times of U. S. stocks," Physical Review E, vol. 69, no. 5, Article ID 056107, 2004.

[86] A. Bunde, S. Havlin, J. W. Kantelhardt, T. Penzel, J.-H. Peter, and K. Voigt, "Correlated and uncorrelated regions in heart-rate fluctuations during sleep," Physical Review Letters, vol. 85, no. 17, pp. 3736-3739, 2000.

[87] J. W. Kantelhardt, E. Koscielny-Bunde, H. H. A. Rego, S. Havlin, and A. Bunde, "Detecting long-range correlations with detrended fluctuation analysis," Physica A: Statistical Mechanics and its Applications, vol. 295, no. 3-4, pp. 441-454, 2001.

[88] K. Hu, P. C. Ivanov, Z. Chen, P. Carpena, and H. E. Stanley, "Effect of trends on detrended fluctuation analysis," Physical Review E, vol. 64, no. 1, Article ID 011114, 2001.

[89] A. Bashan, R. Bartsch, J. W. Kantelhardt, and S. Havlin, "Comparison of detrending methods for fluctuation analysis," Physica A: Statistical Mechanics and its Applications, vol. 387, no. 21, pp. 5080-5090, 2008.

[90] V. I. Keilis-Borok and I. M. Rotwain, "Diagnosis of Time of Increased Probability of strong earthquakes in different regions of the world: algorithm CN," Physics of the Earth and Planetary Interiors, vol. 61, no. 1-2, pp. 57-72, 1990.

[91] V. I. Keilis-Borok and V. G. Kossobokov, "Premonitory activation of earthquake flow: algorithm M8," Physics of the Earth and Planetary Interiors, vol. 61, no. 1-2, pp. 73-83, 1990.

[92] T. Fawcett, "An introduction to ROC analysis," Pattern Recognition Letters, vol. 27, no. 8, pp. 861-874, 2006.

[93] S. J. Mason and N. E. Graham, "Areas beneath the relative operating characteristics (ROC) and relative operating levels (ROL) curves: statistical significance and interpretation," Quarterly Journal of the Royal Meteorological Society, vol. 128, no. 584, pp. 2145-2166, 2002.

[94] H. B. Mann and D. R. Whitney, "On a test of whether one of two random variables is stochastically larger than the other," The Annals of Mathematical Statistics, vol. 18, no. 1, pp. 50-60, 1947.

[95] N. V. Sarlis and S.-R. G. Christopoulos, "Visualization of the significance of Receiver Operating Characteristics based on confidence ellipses," Computer Physics Communications, vol. 185, no. 3, pp. 1172-1176, 2014.

[96] J. Davidsen and A. Green, "Are earthquake magnitudes clustered?" Physical Review Letters, vol. 106, no. 10, Article ID 108502, 2011.

[97] Z. Peng, J. E. Vidale, and H. Houston, "Anomalous early aftershock decay rate of the 2004 Mw6.0 Parkfield, California, earthquake," Geophysical Research Letters, vol. 33, no. 17, Article ID L17307, 2006.

[98] Z. Peng, J. E. Vidale, M. Ishii, and A. Helmstetter, "Seismicity rate immediately before and after main shock rupture from high-frequency waveforms in Japan," Journal of Geophysical Research: Solid Earth, vol. 112, no. B3, 2007.

[99] Z. Peng and P. Zhao, "Migration of early aftershocks following the 2004 Parkfield earthquake," Nature Geoscience, vol. 2, no. 12, pp. 877-881, 2009. 
[100] A. M. Dziewoński, T. Chou, and J. H. Woodhouse, "Determination of earthquake source parameters from waveform data for studies of global and regional seismicity," Journal of Geophysical Research: Solid Earth, vol. 86, no. B4, pp. 2825-2852, 1981.

[101] G. Ekström, M. Nettles, and A. Dziewoński, "The global CMT project 2004-2010: centroid-moment tensors for 13,017 earthquakes," Physics of the Earth and Planetary Interiors, vol. 1, pp. 200-201, 2012.

[102] A. Helmstetter, Y. Y. Kagan, and D. D. Jackson, "Importance of small earthquakes for stress transfers and earthquake triggering," Journal of Geophysical Research: Solid Earth, vol. 110, no. B5, Article ID B05S08, 2005.

[103] A. Helmstetter, Y. Y. Kagan, and D. D. Jackson, "Comparison of short-term and time-independent earthquake forecast models for Southern California," Bulletin of the Seismological Society of America, vol. 96, no. 1, pp. 90-106, 2006.

[104] Y. Ogata, Statistical Analysis of Seismicity-Updated Version (SASeis2006), Computer Science Monographs No. 33, The Institute of Statistical Mathematics, Tokyo, Japan, 2006.

[105] Y. Ogata, "Statistical Models for Earthquake occurrences and residual analysis for point processes," Journal of the American Statistical Association, vol. 83, no. 401, pp. 9-27, 1988.

[106] J. Zhuang and S. Touati, Theme V-Models and Techniques for Analyzing Seismicity: Stochastic Simulation of Earthquake Catalogs, 2015, http://www.corssa.org.

[107] Y. Ogata and K. Katsura, Fortran Programs for Statistical Analysis of Series of Events, Computer Science Monograph, No. 22/23, The Institute of Statistical Mathematics, Tokyo, Japan, 1985.

[108] T. Utsu and Y. Ogata, "Computer program package: Statistical Analysis of point processes for Seismicity, SASeis," in IASPEI, J. Healy, V. Keilis-Borok, and W. Lee, Eds., vol. 6, pp. 13-94, Seismological Society of America, El Cerrito, Calif, USA, 1997.

[109] P. A. Reasenberg and L. M. Jones, "Earthquake hazard after a mainshock in California," Science, vol. 243, no. 4895, pp. 11731176, 1989.

[110] T. Omi, Y. Ogata, Y. Hirata, and K. Aihara, "Forecasting large aftershocks within one day after the main shock," Scientific Reports, vol. 3, article 2218, 2013.

[111] E. Lippiello, C. Godano, and L. De Arcangelis, "Dynamical scaling in branching models for seismicity," Physical Review Letters, vol. 98, no. 9, Article ID 098501, 2007.

[112] E. Lippiello, C. Godano, and L. de Arcangelis, "The earthquake magnitude is influenced by previous seismicity," Geophysical Research Letters, vol. 39, no. 5, Article ID L05309, 2012.

[113] E. Lippiello, L. de Arcangelis, and C. Godano, "Influence of time and space correlations on earthquake magnitude," Physical Review Letters, vol. 100, no. 3, Article ID 038501, 2008.

[114] T. Schreiber and A. Schmitz, "Improved surrogate data for nonlinearity tests," Physical Review Letters, vol. 77, no. 4, pp. 635-638, 1996.

[115] R. Hegger, H. Kantz, and T. Schreiber, "Practical implementation of nonlinear time series methods: the TISEAN package," Chaos, vol. 9, no. 2, pp. 413-435, 1999.

[116] H. Kantz and T. Schreiber, Nonlinear time series analysis, Cambridge University Press, Cambridge, UK, Second edition, 2004.

[117] C. Tsallis, Introduction to Nonextensive Statistical Mechanics, Springer, Berlin, Germany, 2009. 


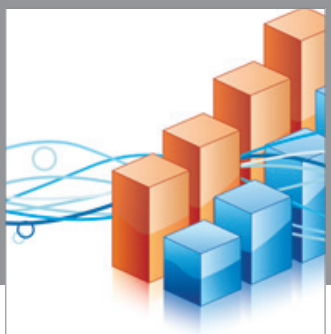

Advances in

Operations Research

vatem alat4

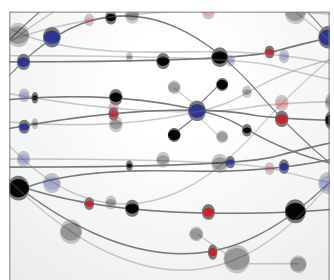

\section{The Scientific} World Journal
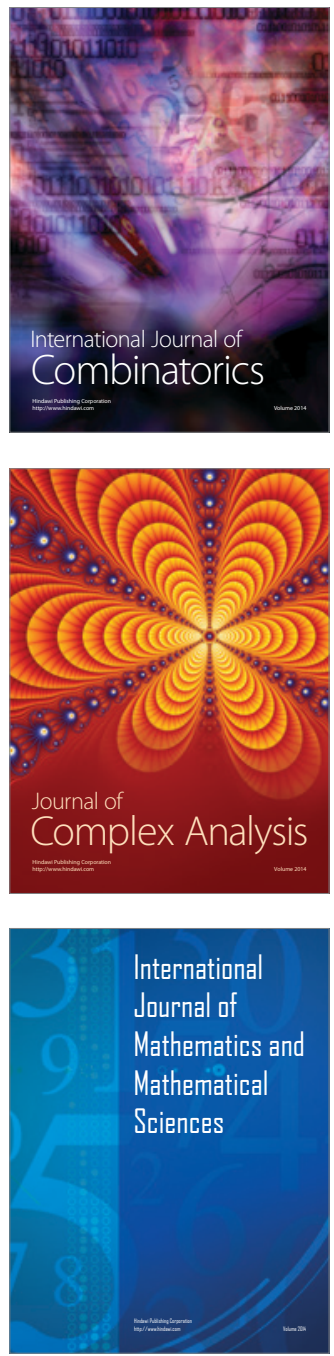
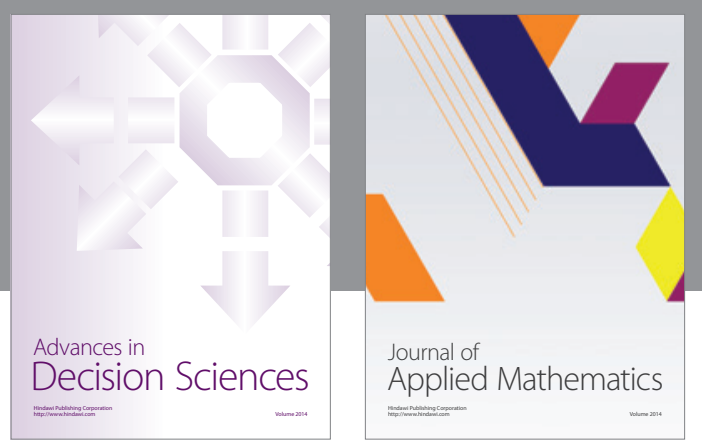

Algebra

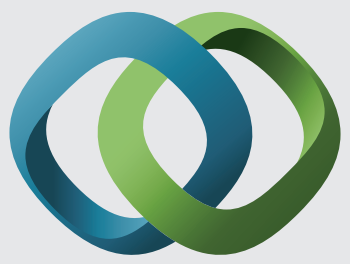

\section{Hindawi}

Submit your manuscripts at

https://www.hindawi.com
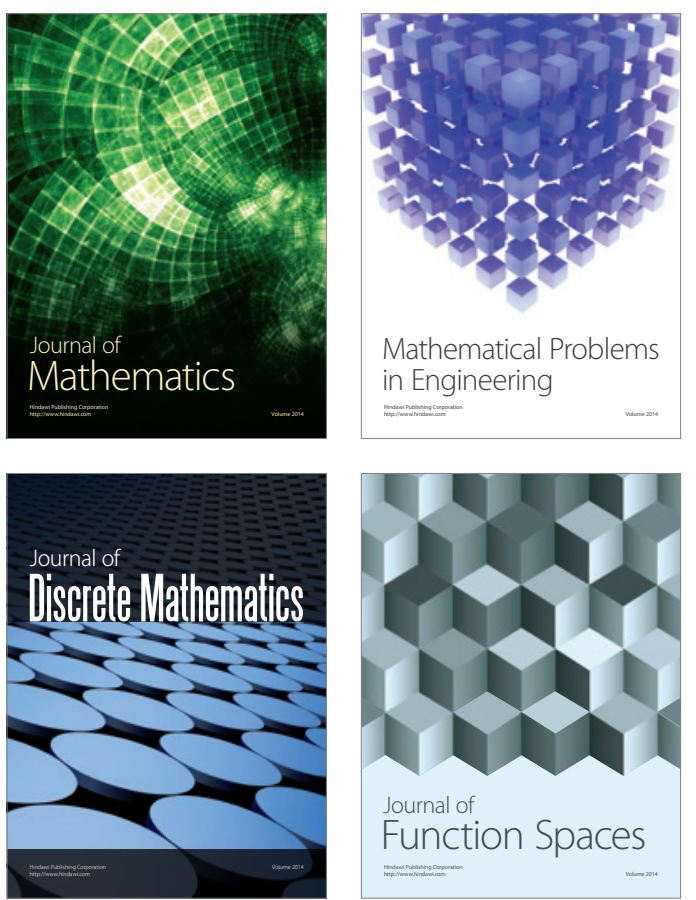

Mathematical Problems in Engineering
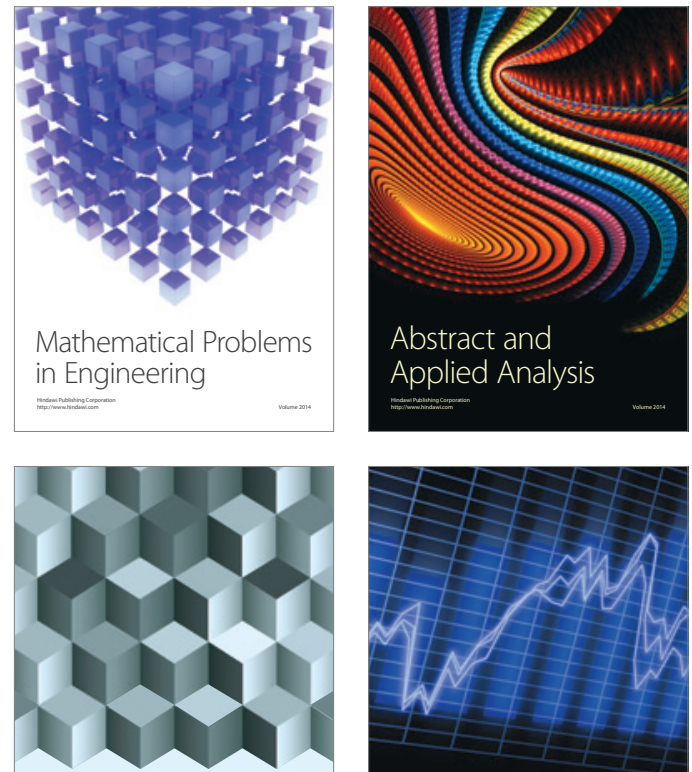

Journal of

Function Spaces

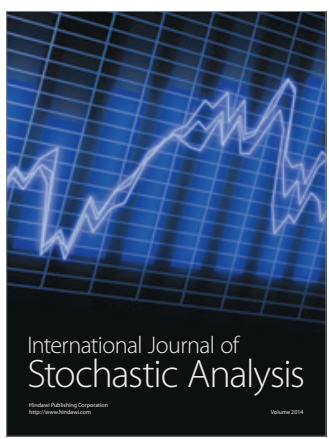

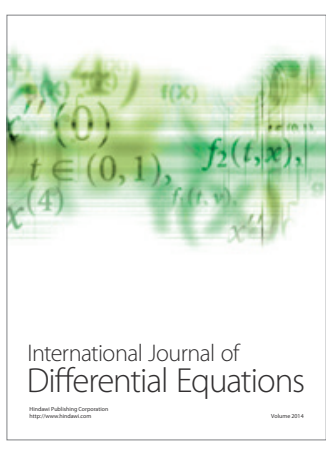
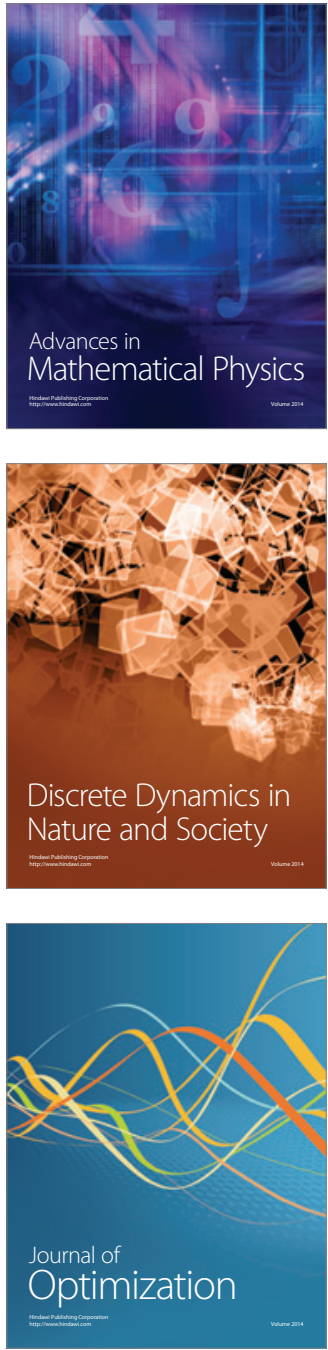